\title{
SPECTRAL STABILITY FOR A CLASS OF FOURTH ORDER STEKLOV PROBLEMS UNDER DOMAIN PERTURBATIONS
}

\author{
ALBERTO FERRERO, PIER DOMENICO LAMBERTI
}

\begin{abstract}
We study the spectral stability of two fourth order Steklov problems upon domain perturbation. One of the two problems is the classical DBS - Dirichlet Biharmonic Steklov - problem, the other one is a variant. Under a comparatively weak condition on the convergence of the domains, we prove the stability of the resolvent operators for both problems, which implies the stability of eigenvalues and eigenfunctions. The stability estimates for the eigenfunctions are expressed in terms of the strong $H^{2}$-norms. The analysis is carried out without assuming that the domains are star-shaped. Our condition turns out to be sharp at least for the variant of the DBS problem. In the case of the DBS problem, we prove stability of a suitable Dirichlet-to-Neumann type map under very weak conditions on the convergence of the domains and we formulate an open problem. As bypass product of our analysis, we provide some stability and instability results for Navier and Navier-type boundary value problems for the biharmonic operator.
\end{abstract}

Keywords: Biharmonic operators, Steklov boundary conditions, spectral stability, domain perturbation, Dirichlet-to-Neumann maps.

2000 Mathematics Subject Classification: 35J40, 35B20, 35P15

\section{INTRODUCTION}

In this paper we consider a class of Steklov problems and related spectral properties. By Steklov problems we mean a class of elliptic problems with parameters in the boundary conditions. This denomination is motivated by [35] where the classical Steklov problem

$$
\begin{cases}\Delta u=0, & \text { in } \Omega, \\ u_{\nu}=\lambda u, & \text { on } \partial \Omega,\end{cases}
$$

was introduced. We refer to the recent paper [25] for a survey on this subject. Here we are interested in the study of a class of elliptic equations with the biharmonic operator subject to Steklov type boundary conditions. Such kind of conditions for the biharmonic operator can be already found in the following works [22, 27, 28, 29, 32]. In those papers the attention is mainly focused on the first eigenvalue of the fourth order Steklov problem and its dependence on the domain. To be more clear, let us introduce the classical DBS Dirichlet Biharmonic Steklov - problem. Let $\Omega \subset \mathbb{R}^{N}(N \geq 2)$ be a bounded domain (i.e., connected open set) with sufficiently regular boundary. Consider the fourth order problem

$$
\begin{cases}\Delta^{2} u=0, & \text { in } \Omega, \\ u=0, & \text { on } \partial \Omega, \\ \Delta u-d u_{\nu}=0, & \text { on } \partial \Omega,\end{cases}
$$

where $u_{\nu}$ denotes the normal derivative on the boundary and $d$ is a real parameter. By a solution of (2) we mean a function $u \in H^{2}(\Omega) \cap H_{0}^{1}(\Omega)$ such that

$$
\int_{\Omega} \Delta u \Delta v d x=d \int_{\partial \Omega} u_{\nu} v_{\nu} d S, \quad \text { for any } v \in H^{2}(\Omega) \cap H_{0}^{1}(\Omega) .
$$

Clearly (2) is a linear homogeneous problem and hence it always admits the trivial solution. Therefore, it appears reasonable to interpret (2) as an eigenvalue problem with respect to the parameter $d$ : we say that $d$ is an eigenvalue for (2) if (2) admits a nontrivial solution in the sense given in (3). Such a nontrivial solution

Date: March 9, 2021. 
is called eigenfunction for $d$. In the sequel, we will refer to (2) as the Steklov problem. It is well-known that the eigenvalues of (2) are strictly positive and that the first eigenvalue $d_{1}$ admits the following variational characterization

$$
d_{1}=\inf _{v \in\left(H^{2}(\Omega) \cap H_{0}^{1}(\Omega)\right) \backslash H_{0}^{2}(\Omega)} \frac{\int_{\Omega}|\Delta v|^{2} d x}{\int_{\partial \Omega} v_{\nu}^{2} d S} .
$$

In [29, 32] the authors study the isoperimetric properties of $d_{1}$ and in [22] it is proved that $d_{1}$ is the sharp constant for $L^{2}$ a priori estimates for solutions of the (second order) Laplace equation under nonhomogeneous Dirichlet boundary conditions, see also [27, 28].

From a physical point of view, it is known that in the two dimensional case $N=2$, Steklov boundary conditions for the biharmonic operator have a natural interpretation in modeling the vibrations of a hinged plate. For more details see for example [14, 15, 21, 23, 24, 30].

In the last years, the Steklov boundary conditions for the biharmonic operator were studied in [8, 24] from the point of view of the positivity preserving property for nonhomogeneous biharmonic equations. Other recent results were focused on isoperimetric properties of $d_{1}$ and on related shape optimization problems, see [2, 13, 14, 21]. More precisely from these papers, among many other results, we know that, among all convex domains of fixed volume, there exists an optimal domain which minimizes $d_{1}$ and that this domain is not the ball: for example in dimension $N=2$ the square is better than the disk. It is still an open question, even in dimension $N=2$, which is the optimal shape among convex domains of fixed volume. We also quote the recent paper [7] where the eigenvalues of (2) are used to provide the singular value decomposition for the Poisson kernel of the Laplace operator on bounded domains.

For its physical interpretation and for its relevance in the study of positivity preserving properties, in our paper we also consider the following Steklov type problem

$$
\begin{cases}\Delta^{2} u=0, & \text { in } \Omega, \\ u=0, & \text { on } \partial \Omega, \\ \Delta u-K(x) u_{\nu}-\delta u_{\nu}=0, & \text { on } \partial \Omega\end{cases}
$$

where $K$ denotes the mean curvature of the boundary, i.e. the sum of the principal curvatures and $\delta$ is a real parameter. As one can see for example from [24], when $N=2$, the curvature of the boundary naturally comes out when we consider the model of a hinged plate. Problem (5) admits the following variational formulation: we say that $u \in H^{2}(\Omega) \cap H_{0}^{1}(\Omega)$ is a weak solution of (5) if

$$
\int_{\Omega} D^{2} u: D^{2} v d x=\delta \int_{\partial \Omega} u_{\nu} v_{\nu} d S \quad \text { for any } v \in H^{2}(\Omega) \cap H_{0}^{1}(\Omega)
$$

where $D^{2} u, D^{2} v$ denote the Hessian matrices of $u, v$ respectively and $D^{2} u: D^{2} v:=\sum_{i, j=1}^{N} \frac{\partial^{2} u}{\partial x_{i} \partial x_{j}} \frac{\partial^{2} v}{\partial x_{i} \partial x_{j}}$. In the sequel we also write $\left|D^{2} u\right|^{2}:=\sum_{i, j=1}^{N}\left(\frac{\partial^{2} u}{\partial x_{i} \partial x_{j}}\right)^{2}$. For more details on the correlation between (5) and (6) see the proof of [19, Proposition 5] and combine it with the identity $\Delta u=u_{\nu \nu}+K(x) u_{\nu}$ on $\partial \Omega$ (see [34, (4.68), p. 62]) valid for any smooth enough function $u$ vanishing on $\partial \Omega$. Here $u_{\nu \nu}$ denotes the second order normal derivative, i.e. $u_{\nu \nu}=\sum_{i, j=1}^{N} \frac{\partial^{2} u}{\partial x_{i} \partial x_{j}} \nu_{i} \nu_{j}$ with $\nu=\left(\nu_{1}, \ldots, \nu_{N}\right)$.

Similarly to problem (2), also problem (5) may be interpreted as an eigenvalue problem with respect to the parameter $\delta$ : we say that $\delta$ is an eigenvalue of (5) if (5) admits a nontrivial solution in the sense given in (6). In the sequel, we refer to (5) as the modified Steklov problem.

It is known that the set of the eigenvalues of (2) is a countable set of isolated positive numbers which may be ordered in an increasing sequence diverging to $+\infty$. We choose the usual notation of repeating each eigenvalue as many times as its multiplicity:

$$
0<d_{1}<d_{2} \leq \cdots \leq d_{n} \leq \cdots
$$

As one can see from (7) we wrote $d_{1}<d_{2}$ in order to emphasize the fact that the first eigenvalue $d_{1}$ is simple. We quote the papers [13, 21] for more details on these and further properties of the eigenvalues of (2). 
In a completely similar way, it can be proved that also the set of the eigenvalues of (5) is a countable set of isolated numbers which may be ordered in an increasing sequence diverging to $+\infty$ and moreover the least eigenvalue is strictly positive. For the eigenvalues of (5) we use a notation analogous to (7):

$$
0<\delta_{1} \leq \delta_{2} \leq \cdots \leq \delta_{n} \leq \cdots
$$

Here we are interested in the study of the stability of the eigenvalues and eigenfunctions of (2) and (5) with respect to domain perturbation. We note that the stability of the eigenvalues of the classical second order problem (10 has been discussed in [10] as well as in [37] which is actually concerned with the stability of the so-called Dirichlet-to-Neumann maps.

More precisely if $\left\{\Omega_{\varepsilon}\right\}_{0<\varepsilon \leq \varepsilon_{0}}$ is a family of domains which converges to a domain $\Omega$ in a suitable sense, denoting by $\left\{d_{n}^{\varepsilon}\right\}_{n=1}^{\infty},\left\{d_{n}\right\}_{n=1}^{\infty}$ the eigenvalues of the Steklov problem in $\Omega_{\varepsilon}$ and $\Omega$ respectively, and by $\left\{\delta_{n}^{\varepsilon}\right\}_{n=1}^{\infty}\left\{\delta_{n}\right\}_{n=1}^{\infty}$ the eigenvalues of the modified Steklov problem in $\Omega_{\varepsilon}$ and $\Omega$ respectively, we say that we have stability for the eigenvalues of each of the two problems, if for any $n \geq 1, d_{n}^{\varepsilon} \rightarrow d_{n}$ and $\delta_{n}^{\varepsilon} \rightarrow \delta_{n}$ as $\varepsilon \rightarrow 0$. We are also interested in what we will call spectral convergence of $S_{\Delta, \varepsilon}$ to $S_{\Delta}$ and of $S_{D^{2}, \varepsilon}$ to $S_{D^{2}}$, where $S_{\Delta, \varepsilon}, S_{\Delta}$ denote the linear operators associated to (2) respectively in $\Omega_{\varepsilon}$ and $\Omega$, and $S_{D^{2}, \varepsilon}, S_{D^{2}}$ denote the linear operators associated to (5) respectively in $\Omega_{\varepsilon}$ and $\Omega$. See Section 3.1 for the precise definitions of these operators.

Basically, these operators are the resolvent operators associated with problems (2), (5) and their spectral convergence is obtained by proving their compact convergence which is a well-known notion of convergence implying the convergence of eigenvalues and eigenfunctions. Unfortunately, since the underlying function spaces depend on $\varepsilon$, we cannot directly use the standard notion of compact convergence, but we need to use suitable 'connecting systems' which allow to pass from the varying Hilbert spaces defined on $\Omega_{\varepsilon}$ to the limiting fixed Hilbert space defined on $\Omega$. Namely, we use a number of notions and results which go back to the works of F. Stummel [36] and G. Vainniko [38] and which have been further implemented in [3, 17]. See also the recent paper [9]. In particular we use the notion of $E$-compact convergence. These notions are discussed in detail in Section 2.1 .

In order to prove the $E$-compact convergence of the operators under consideration, we consider domains $\Omega_{\varepsilon}, \Omega$ belonging to uniform classes of domains with $C^{1,1}$ boundaries - see Definition 2.6- and we require that the boundaries of $\Omega_{\varepsilon}$ converge to the boundary of $\Omega$ in the sense of (21). Condition (21) has been introduced in [4] and turns out to be a sharp condition in the analysis of domain perturbation problems for fourth order operators. Note that if the boundaries of $\Omega_{\varepsilon}$ have uniformly bounded $C^{1,1}$-norms and converge to the boundary of $\Omega$ in $C^{1}$ then condition (21) is satisfied. However, it is important to note that condition (21) allows to deal also with boundaries whose $C^{1,1}$-norms are not uniformly bounded with respect to $\varepsilon$.

Condition (21) allows to construct in Section 3.2 a linear continuous operator $E_{\varepsilon}: H^{2}(\Omega) \cap H_{0}^{1}(\Omega) \rightarrow$ $H^{2}\left(\Omega_{\varepsilon}\right) \cap H_{0}^{1}\left(\Omega_{\varepsilon}\right)$ and to define the notion of $E$-convergence for a sequence $u_{\varepsilon} \in H^{2}\left(\Omega_{\varepsilon}\right) \cap H^{1}\left(\Omega_{\varepsilon}\right)$ to a function $u \in H^{2}(\Omega) \cap H_{0}^{1}(\Omega)$, that is

$$
\left\|u_{\varepsilon}-E_{\varepsilon} u\right\|_{H^{2}\left(\Omega_{\varepsilon}\right)} \rightarrow 0, \text { as } \varepsilon \rightarrow 0 .
$$

The operators $E_{\varepsilon}$ are constructed by pasting together suitable pull-back operators defined by means of appropriate local diffeomorphisms. Importantly, the operators $E_{\varepsilon}$ enjoy the following property: for any $\varepsilon>0$ there exists an open set $K_{\varepsilon} \subset \Omega \cap \Omega_{\varepsilon}$ such that $\left(E_{\varepsilon} u\right)(x)=u(x)$ for all $x \in K_{\varepsilon}$ and such that $\left|\left(\Omega_{\varepsilon} \cup \Omega\right) \backslash K_{\varepsilon}\right| \rightarrow 0$ as $\varepsilon \rightarrow 0$. Here and in the sequel $|A|$ denotes the Lebesgue measure of any measurable set $A \subset \mathbb{R}^{N}$. This implies the more familiar strong convergence

$$
\left\|u_{\varepsilon}-u\right\|_{H^{2}\left(\Omega_{\varepsilon} \cap \Omega\right)} \rightarrow 0, \text { as } \varepsilon \rightarrow 0 .
$$

Moreover, it also implies

$$
\left\|\nabla u_{\varepsilon}-E_{\varepsilon} \nabla u\right\|_{L^{2}\left(\partial \Omega_{\varepsilon}\right)} \rightarrow 0, \text { as } \varepsilon \rightarrow 0,
$$

and we note that $E_{\varepsilon} \nabla u_{\mid \partial \Omega_{\varepsilon}}$ is nothing but a natural transplantation of $u$ from $\partial \Omega$ to $\partial \Omega_{\varepsilon}$ (which differs from an exact pull-back by a minor deformation at the intersections of different local charts describing the boundaries, a deformation which vanishes as $\varepsilon \rightarrow 0$ ). For more details on (9)-(10) see Proposition 3.11. 
One of the main results of the paper is Theorem 3.2 where it is proved that condition (21) implies the $E$ compact convergence of $S_{\Delta, \varepsilon}$ to $S_{\Delta}$ and of $S_{D^{2}, \varepsilon}$ to $S_{D^{2}}$ as $\varepsilon \rightarrow 0$. On the base of the general Theorem 2.5, this implies the spectral convergence of $S_{\Delta, \varepsilon}$ to $S_{\Delta}$ and of $S_{D^{2}, \varepsilon}$ to $S_{D^{2}}$, hence the convergence of the eigenvalues and the $E$-convergence of the eigenfunctions in the sense of Theorem 2.5. In particular the eigenfunctions converge in the sense of (9) and (10).

We note that our domains are not required to be star-shaped as in [37] (which is concerned with second order operators) and that removing such an assumption is not straightforward. In fact, here we only assume that the domains under consideration belong to a uniform class of domains defined by means of the same atlas and this leads to a number of technical difficulties that are overcome in Section 3. We believe that Section 3 gives a partial answer (in the case of fourth order operators) to the question raised in the introduction of [37] where the authors suggest the problem of studying the convergence of the resolvent operators of second order Steklov problems on arbitrary Lipschitz domains.

In Section 4 we analyze the optimality of condition (21). In the spirit of [4], we consider the case of a domain of the form $\Omega=W \times(-1,0)$ where $W$ is a cuboid or a bounded domain in $\mathbb{R}^{N-1}$ of class $C^{1,1}$ and we assume that the perturbed domain $\Omega_{\varepsilon}$ is given by

$$
\Omega_{\varepsilon}=\left\{\left(x^{\prime}, x_{N}\right): x^{\prime} \in W,-1<x_{N}<g_{\varepsilon}\left(x^{\prime}\right)\right\}
$$

where $g_{\varepsilon}\left(x^{\prime}\right)=\varepsilon^{\alpha} b\left(x^{\prime} / \varepsilon\right)$ for any $x^{\prime} \in W$ and $b: \mathbb{R}^{N-1} \rightarrow[0,+\infty)$ is a nonconstant $Y$-periodic function where $Y=\left(-\frac{1}{2}, \frac{1}{2}\right)^{N-1}$ is the unit cell in $\mathbb{R}^{N-1}$. We denote by $\Gamma_{\varepsilon}$ and $\Gamma$ the sets

$$
\Gamma_{\varepsilon}:=\left\{\left(x^{\prime}, g_{\varepsilon}\left(x^{\prime}\right)\right): x^{\prime} \in W\right\} \quad \text { and } \quad \Gamma:=W \times\{0\} .
$$

It appears that for $\alpha>3 / 2$ condition (21) is satisfied hence we have spectral convergence of $S_{\Delta, \varepsilon}$ to $S_{\Delta}$ and of $S_{D^{2}, \varepsilon}$ to $S_{D^{2}}$, see Corollary 4.1. For $\alpha<3 / 2$ condition (21) is not satisfied and we have a degeneration phenomenon. Namely, for $1 \leq \alpha<3 / 2$ we have spectral convergence of the operator $S_{D^{2}, \varepsilon}$ to $S_{D^{2}, \Gamma}$ where $S_{D^{2}, \Gamma}$ is an operator encoding Dirichlet boundary conditions on $\Gamma$, that is $u=u_{\nu}=0$ on $\partial \Gamma$, see Theorem 4.1. For $\alpha=3 / 2$, we have spectral convergence to another operator which encodes the appearance of a strange term in the boundary conditions, see Theorem 4.5. We note that in order to simplify our analysis in the case $\alpha=3 / 2$, we impose Dirichlet boundary conditions on $\partial \Omega_{\varepsilon} \backslash \Gamma_{\varepsilon}$ and $\partial \Omega \backslash \Gamma$.

We also note that imposing Dirichlet boundary conditions on $\partial \Omega_{\varepsilon} \backslash \Gamma_{\varepsilon}$ and $\partial \Omega \backslash \Gamma$ allows to view the trichotomy discussed above in a rather transparent way. Namely, under Dirichlet boundary conditions on $\partial \Omega_{\varepsilon} \backslash \Gamma_{\varepsilon}$ and $\partial \Omega \backslash \Gamma$ we have that:

(i) If $\alpha>3 / 2$ then $\delta_{n}(\varepsilon) \rightarrow \delta_{n}(0)$, as $\varepsilon \rightarrow 0$.

(ii) If $\alpha=3 / 2$ then $\delta_{n}(\varepsilon) \rightarrow \delta_{n}(0)+\gamma$ as $\varepsilon \rightarrow 0$, where $\gamma>0$ is the strange term defined by (134).

(iii) If $1 \leq \alpha<3 / 2$ then $\delta_{n}(\varepsilon) \rightarrow \infty$, as $\varepsilon \rightarrow 0$.

The strange term $\gamma$ is the same term found in [4] in the analysis of the Babuska Paradox and is referred to as 'the strange curvature' in [4].

The analysis of the cases $\alpha \leq 3 / 2$ is carried out in Section 4 only for problem (5) since problem (2) presents severe difficulties in these cases. In order to overcome these difficulties, in Section 5 we change our point of view and we recast the eigenvalue problem (2) as an eigenvalue problem for a suitable Dirichlet-toNeumann-type map. Considering our boundary conditions, we find it natural to call such a map Neumannto-Navier map, and actually we find it simpler to consider its inverse which we call Navier-to-Neumann map and we denote by $\mathcal{N}_{\Omega}$. Since we aim at relaxing the boundary regularity assumptions, we are forced to work with the space $H(\Delta, \Omega) \cap H_{0}^{1}(\Omega)$ and we find it convenient to consider the normal derivatives of the functions in such a space as elements of $H^{-1 / 2}(\partial \Omega)$ although they belong to $L^{2}(\partial \Omega)$ see [7, Thm. 3.2] (recall that the space $H(\Delta, \Omega)$ is the space of functions in $L^{2}(\Omega)$ with distributional Laplacian in $L^{2}(\Omega)$ ). In this way, our Navier-to-Neumann map $\mathcal{N}_{\Omega}$ can be considered as a map from $H^{1}(\Omega) / H_{0}^{1}(\Omega)$ to its dual and problem (2) can be recast in the form $\mathcal{N}_{\Omega} f=d^{-1} J_{0} f$ where $J_{0}$ is defined by $\left\langle J_{0} f, v\right\rangle:=\int_{\partial \Omega} f v d S$ for all $f, v \in H^{1}(\Omega) / H_{0}^{1}(\Omega)$. It is interesting to note that this weak formulation of problem (2) makes sense under the sole assumption that $\Omega$ is a bounded domain of class $C^{0,1}$. We note that, in order to avoid some technicalities which would not add much to the results of Section 5 , we shall actually avoid using the quotient 
spaces and we shall consider the Navier-to-Neumann $\mathcal{N}_{\Omega}$ directly as map from $H^{1}(\Omega)$ to its dual, which has also its own interest, see Remark 6 .

Thus we study the behaviour of the maps $\mathcal{N}_{\Omega_{\varepsilon}}$ as $\varepsilon \rightarrow 0$ and in Theorem 5.6 we prove their $E E^{*}$ convergence to $\mathcal{N}_{\Omega}$ under weak conditions on the convergence of domains. Here the $E E^{*}$-convergence is a natural type of convergence for operators acting from a family of spaces to the corresponding duals and is based on two connecting systems $E_{\varepsilon}$ and $E_{\varepsilon}^{*}$ ( $E_{\varepsilon}$ is now defined by means of a standard extension operator for the space $H^{1}(\Omega)$ ), see Definition 5.4. In Theorem 5.7 we also prove a compactness result for the convergence of $\mathcal{N}_{\Omega_{\varepsilon}}$ under an additional assumption on the convergence of $\Omega_{\varepsilon}$.

Unfortunately, the compact convergence result obtained in Theorem 5.7 is not sufficient to prove a stability result for the spectrum of the Steklov problem (2) under such very weak assumptions on the convergence of $\Omega_{\varepsilon}$. Indeed, in order to apply [38, Theorem 6.1], the regular convergence condition 1) in [38, Section 6] has to be satisfied by the family of operators $\left\{\mathcal{N}_{\Omega_{\varepsilon}}-\lambda\left(J_{0}\right)_{\varepsilon}\right\}_{\varepsilon}$ for any $\lambda \in \Lambda$, where $\Lambda$ is a suitable bounded set in the complex plane, and this does not seem the case, not even if we consider these operators on the quotient spaces and adjust the corresponding connecting systems. Taking into account this issue we suggest the Open Problem 5.1

As a bypass product of the analysis carried out in Section 5, we prove stability and instability results for Navier and Navier-type problems, see Theorems 5.3 and 5.8 .

The paper is organized as follows. In Section 2 we discuss some preliminary results, in particular we discuss the notion of $E$-convergence and our functional setting. In Section 3 we prove the main stability result of the paper under the assumption that condition (21) holds. In Section 4 we discuss the optimality of condition (21). In Section 5 we relax the assumptions on the convergence of the domains and we prove a stability result for the above mentioned Navier-to-Neumann map; moreover, we discuss the stability and instability of Navier and Navier-type problems.

\section{PRELIMINARIES AND NOTATION}

2.1. A general approach to spectral stabilty. The study of all spectral problems considered in this paper will be reduced to the study of convenient non-negative compact self-adjoint operators in Hilbert spaces.

A natural way to do so is to consider appropriate modified versions of the classical Dirichlet-to-Neumann map densely defined in $L^{2}(\partial \Omega)$ and to consider their resolvents which are defined on the whole of $L^{2}(\partial \Omega)$. For our fourth order Steklov problems, it is natural to talk about Neumann-to-Navier map and its inverse will be called here Navier-to-Neuman map. Then one note that the eigenvalues of those Navier-to-Neumann maps are in fact the reciprocals of the eigenvalues of our differential problems. One drawback of using Navier-toNeumann maps consists in the fact that eventually they provide information concerning the convergence of the (normal derivatives of the) eigenfunctions considered as elements of $L^{2}(\partial \Omega)$. In order to provide global information about the behaviour of the eigenfunctions in $\Omega$, in the regular cases we shall actually consider appropriate realisations of the resolvents in $H^{2}(\Omega)$ which will allow to get stronger stability results involving the norm of $H^{2}(\Omega)$.

Thus, our spectral problems will be considered from two different points of view depending on the regularity of the domain perturbations. In any case, both methods lead to the study of suitable families $\left\{B_{\varepsilon}\right\}_{0<\varepsilon \leq \varepsilon_{0}}$ of non-negative compact self-adjoint operators defined in Hilbert spaces $\mathcal{H}_{\varepsilon}$ associated with the domains $\Omega_{\varepsilon}$. It is then important to study the convergence of those operators $B_{\varepsilon}$ as $\varepsilon \rightarrow 0$. To do so, we shall use the notion of $E$-convergence which we now recall. We follow the approach of [38], further developed in [3], [17.

In the spirit of [3] we denote by $\mathcal{H}_{\varepsilon}$ a family of Hilbert spaces for $\varepsilon \in\left[0, \varepsilon_{0}\right]$ and we assume that there exists a family of linear operators $E_{\varepsilon}: \mathcal{H}_{0} \rightarrow \mathcal{H}_{\varepsilon}$ such that

$$
\left\|E_{\varepsilon} u\right\|_{\mathcal{H}_{\varepsilon}} \stackrel{\varepsilon \rightarrow 0}{\longrightarrow}\|u\|_{\mathcal{H}_{0}}, \quad \text { for all } u \in \mathcal{H}_{0} .
$$

Definition 2.1. We say that a family $\left\{u_{\varepsilon}\right\}_{0<\varepsilon \leq \varepsilon_{0}}$, with $u_{\varepsilon} \in \mathcal{H}_{\varepsilon}$, E-converges to $u \in \mathcal{H}_{0}$ if $\left\|u_{\varepsilon}-E_{\varepsilon} u\right\|_{\mathcal{H}_{\varepsilon}} \rightarrow$ 0 as $\varepsilon \rightarrow 0$. We write this as $u_{\varepsilon} \stackrel{E}{\longrightarrow} u$. 
Definition 2.2. Let $\left\{B_{\varepsilon} \in \mathcal{L}\left(\mathcal{H}_{\varepsilon}\right): \varepsilon \in\left(0, \varepsilon_{0}\right]\right\}$ be a family of linear and continuous operators. We say that $\left\{B_{\varepsilon}\right\}_{0<\varepsilon \leq \varepsilon_{0}}$ converges to $B_{0} \in \mathcal{L}\left(\mathcal{H}_{0}\right)$ as $\varepsilon \rightarrow 0$ if $B_{\varepsilon} u_{\varepsilon} \stackrel{E}{\longrightarrow} B_{0} u$ whenever $u_{\varepsilon} \stackrel{E}{\longrightarrow} u$. We write this as $B_{\varepsilon} \stackrel{E E}{\longrightarrow} B_{0}$.

Definition 2.3. Let $\left\{u_{\varepsilon}\right\}_{0<\varepsilon \leq \varepsilon_{0}}$ be a family such that $u_{\varepsilon} \in \mathcal{H}_{\varepsilon}$. We say that $\left\{u_{\varepsilon}\right\}_{0<\varepsilon \leq \varepsilon_{0}}$ is precompact if for any sequence $\varepsilon_{n} \rightarrow 0$ there exist a subsequence $\left\{\varepsilon_{n_{k}}\right\}$ and $u \in \mathcal{H}_{0}$ such that $u_{\varepsilon_{n_{k}}} \stackrel{\bar{E}}{\longrightarrow} u$.

Definition 2.4. We say that $\left\{B_{\varepsilon}\right\}_{0<\varepsilon \leq \varepsilon_{0}}$ with $B_{\varepsilon} \in \mathcal{L}\left(\mathcal{H}_{\varepsilon}\right)$ and $B_{\varepsilon}$ compact, converges compactly to a compact operator $B_{0} \in \mathcal{L}\left(\mathcal{H}_{0}\right)$ if $B_{\varepsilon} \stackrel{E E}{\longrightarrow} B_{0}$ and for any family $\left\{u_{\varepsilon}\right\}_{0<\varepsilon \leq \varepsilon_{0}}$ such that $u_{\varepsilon} \in \mathcal{H}_{\varepsilon},\left\|u_{\varepsilon}\right\|_{\mathcal{H}_{\varepsilon}}=1$, we have that $\left\{B_{\varepsilon} u_{\varepsilon}\right\}_{0<\varepsilon \leq \varepsilon_{0}}$ is precompact in the sense of Definition 2.3 We write this as $B_{\varepsilon} \stackrel{C}{\longrightarrow} B_{0}$.

Recall that given a non-negative compact self-adjoint operator $B$ in a infinite dimensional Hilbert space $\mathcal{H}$, the spectrum is a finite or countably infinite set and all non-zero elements of the spectrum are positive eigenvalues of finite multiplicity. In case the spectrum is a countably infinite set, the eigenvalues can be represented by a non-increasing sequence $\mu_{n}, n \in \mathbb{N}$, such that $\lim _{n \rightarrow \infty} \mu_{n}=0$. As customary, we agree to repeat each eigenvalue $\mu_{n}$ in the sequence as many times as its multiplicity. Given a finite set of $m$ eigenvalues $\mu_{n}, \ldots, \mu_{n+m-1}$ with $\mu_{n} \neq \mu_{n-1}$ and $\mu_{n+m-1} \neq \mu_{n+m}$, we call generalized eigenfunction (associated with $\mu_{n}, \ldots, \mu_{n+m-1}$ ) any linear combination of eigenfunctions associated with the eigenvalues $\mu_{n}, \ldots, \mu_{n+m-1}$. Then we have the following theorem which is a simplified rephrased version of [38, Theorem 6.3], see also [3, Theorem 4.10], [6, Theorem 5.1], [17, Theorem 3.3].

Theorem 2.5. Let $\left\{B_{\varepsilon}\right\}_{0<\varepsilon \leq \varepsilon_{0}}$ and $B_{0}$ be non-negative compact self-adjoint operators in the Hilbert spaces $\mathcal{H}_{\varepsilon}, \mathcal{H}_{0}$ respectively. Assume that their eigenvalues are given by the sequences $\mu_{n}(\varepsilon)$ and $\mu_{n}(0), n \in \mathbb{N}$, respectively. If $B_{\varepsilon} \stackrel{C}{\longrightarrow} B_{0}$ then we have spectral convergence of $B_{\varepsilon}$ to $B_{0}$ as $\varepsilon \rightarrow 0$ in the sense that the following statements hold:

(i) For every $n \in \mathbb{N}$ we have $\mu_{n}(\varepsilon) \rightarrow \mu_{n}(0)$ as $\varepsilon \rightarrow 0$.

(ii) If $u_{n}(\varepsilon), n \in \mathbb{N}$, is an orthonormal sequence of eigenfunctions associated with the eigenvalues $\mu_{n}(\varepsilon)$ then there exists a sequence $\varepsilon_{k}, k \in \mathbb{N}$, converging to zero and orthonormal sequence of eigenfunctions $u_{n}(0), n \in \mathbb{N}$ associated with $\mu_{n}(0), n \in \mathbb{N}$ such that $u_{n}\left(\varepsilon_{k}\right) \stackrel{E}{\longrightarrow} u_{n}(0)$.

(iii) Given $m$ eigenvalues $\mu_{n}(0), \ldots, \mu_{n+m-1}(0)$ with $\mu_{n}(0) \neq \mu_{n-1}(0)$ and $\mu_{n+m-1}(0) \neq \mu_{n+m}(0)$ and corresponding orthonormal eigenfunctions $u_{n}(0), \ldots, u_{n+m-1}(0)$, there exist $m$ orthonormal generalized eigenfunctions $v_{n}(\varepsilon), \ldots, v_{n+m-1}(\varepsilon)$ associated with $\mu_{n}(\varepsilon), \ldots, \mu_{n+m-1}(\varepsilon)$ such that $v_{n+i}(\varepsilon) \stackrel{E}{\longrightarrow} u_{n+i}(0)$ for all $i=0,1, \ldots, m-1$.

Remark 1. We mention that an alternative approach to the spectral analysis of operators defined on variable Hilbert spaces has been recently proposed in the book by $O$. Post [33]. In particular, that method allows to obtain stability estimates which can be expressed in terms of suitable estimates for the variation of the resolvent operators. It would be interesting to implement that approach in our setting in particular in the cases where we do not have degeneration phenomena but this would require heavier computations going beyond the scopes of the present paper.

2.2. Classes of domains. In order study the spectral convergence for Steklov eigenvalue problems on varying domains, we shall consider uniform families of domains for which a number of parameters are prescribed. This is done by using the notion of atlas from [16], see also [4, Section 5]. According to [4, 16], given a set $V \subset \mathbb{R}^{N}$ and a number $\delta>0$ we write

$$
V_{\delta}:=\{x \in V: d(x, \partial V)>\delta\} .
$$

Definition 2.6. [16, Definition 2.4] Let $\rho>0, s, s^{\prime} \in \mathbb{N}$ with $s^{\prime}<s$. Let $\left\{V_{j}\right\}_{j=1}^{s}$ be a family of open cuboids (i.e. rotations of rectangle parallelepipeds in $\mathbb{R}^{N}$ ) and $\left\{r_{j}\right\}_{j=1}^{s}$ be a family of rotations in $\mathbb{R}^{N}$. We say that $\mathcal{A}=\left(\rho, s, s^{\prime},\left\{V_{j}\right\}_{j=1}^{s},\left\{r_{j}\right\}_{j=1}^{s}\right)$ is an atlas in $\mathbb{R}^{N}$ with parameters $\rho, s, s^{\prime},\left\{V_{j}\right\}_{j=1}^{s},\left\{r_{j}\right\}_{j=1}^{s}$ briefly an atlas in $\mathbb{R}^{N}$. Moreover, we say that a bounded domain $\Omega$ in $\mathbb{R}^{N}$ belongs to the class $C^{k, \gamma}(\mathcal{A})$ with $k \in \mathbb{N}$ and $\gamma \in[0,1]$ if the following conditions are satisfied: 
(i) $\Omega \subset \cup_{j=1}^{s}\left(V_{j}\right)_{\rho}$ and $\left(V_{j}\right)_{\rho} \cap \Omega \neq \emptyset$ where $\left(V_{j}\right)_{\rho}$ is meant in the sense given in (12);

(ii) $V_{j} \cap \partial \Omega \neq \emptyset$ for $j=1, \ldots, s^{\prime}$ and $V_{j} \cap \partial \Omega=\emptyset$ for $s^{\prime}+1 \leq j \leq s$;

(iii) for $j=1, \ldots, s$ we have

$$
\begin{aligned}
& r_{j}\left(V_{j}\right)=\left\{x \in \mathbb{R}^{N}: a_{i j}<x_{i}<b_{i j}, i=1, \ldots, N\right\}, \quad j=1, \ldots, s ; \\
& r_{j}\left(V_{j} \cap \Omega\right)=\left\{x=\left(x^{\prime}, x_{N}\right) \in \mathbb{R}^{N}: x^{\prime} \in W_{j}, a_{N j}<x_{N}<g_{j}\left(x^{\prime}\right)\right\}, \quad j=1, \ldots, s^{\prime}
\end{aligned}
$$

where $x^{\prime}=\left(x_{1}, \ldots, x_{N-1}\right), W_{j}=\left\{x^{\prime} \in \mathbb{R}^{N-1}: a_{i j}<x_{i}<b_{i j}, i=1, \ldots, N-1\right\}$ and the functions $g_{j} \in C^{k, \gamma}\left(\overline{W_{j}}\right)$ for any $j \in 1, \ldots, s^{\prime}$ with $k \in \mathbb{N} \cup\{0\}$ and $0 \leq \gamma \leq 1$. Moreover, for $j=1, \ldots, s^{\prime}$ we assume that $a_{N j}+\rho \leq g_{j}\left(x^{\prime}\right) \leq b_{N j}-\rho$, for all $x^{\prime} \in \overline{W_{j}}$.

Finally we say that $\Omega$ if of class $C^{k, \gamma}$ if it is of class $C^{k, \gamma}(\mathcal{A})$ for some atlas $\mathcal{A}$. In the sequel $C^{k, 0}$ will be simply denoted by $C^{k}$.

2.3. Function spaces. Let $\Omega$ be a domain in $\mathbb{R}^{N}$. In this paper, we shall often use the standard Sobolev spaces $H^{k}(\Omega)$ and $H_{0}^{k}(\Omega)$ for $k=1,2$. Recall that $H^{k}(\Omega)$ is the space of those functions in $L^{2}(\Omega)$ with weak derivatives up to order $k$ in $L^{2}(\Omega)$, and that $H_{0}^{k}(\Omega)$ is the closure in $H^{k}(\Omega)$ of $C_{c}^{\infty}(\Omega)$. Note that in this paper we assume that $L^{2}(\Omega)$ is made of real-valued functions.

Besides $H^{2}(\Omega)$ we shall also consider the larger space $H(\Delta, \Omega)$ defined by

$$
H(\Delta, \Omega)=\left\{u \in L^{2}(\Omega): \Delta u \in L^{2}(\Omega)\right\},
$$

where $\Delta u$ denotes the distributional Laplacian of $u$. Thus, by definition $\int_{\Omega} u \Delta \varphi d x=\int_{\Omega} \Delta u \varphi d x$ for all $\varphi \in C_{c}^{\infty}(\Omega)$. The space $H(\Delta, \Omega)$ is a natural space associated with the Dirichlet Laplacian which we temporarily denote here by $\Delta_{D}$. Recall that the operator $\Delta_{D}$ is the non-negative self-adjoint operator in $L^{2}(\Omega)$ canonically associated with the quadratic form $\int_{\Omega} \nabla u \nabla \varphi d x$ defined for all $u, \varphi \in H_{0}^{1}(\Omega)$. This means that a function $u \in L^{2}(\Omega)$ belongs to the domain $\operatorname{Dom}\left(\Delta_{D}\right)$ of $\Delta_{D}$ if and only if $u \in H_{0}^{1}(\Omega)$ and there exists $f \in L^{2}(\Omega)$ such that $\int_{\Omega} \nabla u \nabla \varphi d x=\int_{\Omega} f \varphi d x$ for all $\varphi \in H_{0}^{1}(\Omega)$, in which case $-\Delta_{D} u=f$. It follows by these definitions that $\operatorname{Dom}\left(\Delta_{D}\right)=H(\Delta, \Omega) \cap H_{0}^{1}(\Omega)$.

We have the following lemma where by Poincaré inequality we mean the classical inequality $\|u\|_{L^{2}(\Omega)} \leq$ $c\|\nabla u\|_{L^{2}(\Omega)}$ for all $u \in H_{0}^{1}(\Omega)$ and by Poincaré constant we mean the best constant $c$ in that inequality. Recall that the Poincaré inequality holds under general assumptions on $\Omega$, for example if $\Omega$ has finite measure it holds with $c=c_{N}|\Omega|^{\frac{1}{N}}$, or if $\Omega$ is bounded in one direction it holds with $c=c_{N} d$ where $d$ is the diameter in that direction (here the symbol $c_{N}$ is used to denote constants depending only on $N$ ). Moreover, the definition of uniform exterior ball condition is standard, see e.g., [1].

Lemma 2.7. Let $\Omega$ be a domain in $\mathbb{R}^{N}$ such that the Poincaré inequality holds. Then the following statements hold:

(i) For all $u \in H(\Delta, \Omega) \cap H_{0}^{1}(\Omega)$ we have

$$
\|u\|_{L^{2}(\Omega)} \leq c_{\mathcal{P}}^{2}\|\Delta u\|_{L^{2}(\Omega)} \text {, and }\|\nabla u\|_{L^{2}(\Omega)} \leq c_{\mathcal{P}}\|\Delta u\|_{L^{2}(\Omega)},
$$

where $c_{\mathcal{P}}$ denotes the Poincaré constant.

(ii) The space $H(\Delta, \Omega) \cap H_{0}^{1}(\Omega)$ endowed with the scalar product defined by $\int_{\Omega} \Delta u \Delta v d x$ for all $u, v \in$ $H(\Delta, \Omega) \cap H_{0}^{1}(\Omega)$ is a Hilbert space.

(iii) If $\Omega$ is a bounded open set of class $C^{0,1}$ which satisfies the uniform exterior ball condition then $H(\Delta, \Omega) \cap H_{0}^{1}(\Omega)=H^{2}(\Omega) \cap H_{0}^{1}(\Omega)$.

Proof. The second inequality in (i) easily follows by the Poincaré inequality because if $u \in H(\Delta, \Omega) \cap$ $H_{0}^{1}(\Omega)$ then

$$
\int_{\Omega}|\nabla u|^{2} d x=-\int_{\Omega} u \Delta u d x \leq\|u\|_{L^{2}(\Omega)}\|\Delta u\|_{L^{2}(\Omega)} \leq c_{\mathcal{P}}\|\nabla u\|_{L^{2}(\Omega)}\|\Delta u\|_{L^{2}(\Omega)} .
$$

The first inequality in (i) follows by the second inequality combined again with the Poincaré inequality. We now prove statement (ii). It clearly suffices to prove the completeness of the space. Let $u_{n}, n \in \mathbb{N}$ be a sequence in $H(\Delta, \Omega) \cap H_{0}^{1}(\Omega)$ such that $f_{n}:=\Delta u_{n}$ is a Cauchy sequence in $L^{2}(\Omega)$. By $(i)$, also $u_{n}, n \in \mathbb{N}$ 
is a Cauchy sequence in $H_{0}^{1}(\Omega)$. Thus, there exists $f \in L^{2}(\Omega)$ and $u \in H_{0}^{1}(\Omega)$ such that $\Delta u_{n} \rightarrow f$ in $L^{2}(\Omega)$ and $u_{n} \rightarrow u$ in $H_{0}^{1}(\Omega)$ as $n \rightarrow \infty$. Passing to the limit in the equality $\int_{\Omega} \nabla u_{n} \nabla \varphi d x=-\int_{\Omega} f_{n} \varphi d x$ as $n \rightarrow \infty$, we get $\int_{\Omega} \nabla u \nabla \varphi d x=-\int_{\Omega} f \varphi d x$ for all $\varphi \in H_{0}^{1}(\Omega)$ which means that $-\Delta u=f$ hence $u \in H(\Delta, \Omega)$. This completes the proof of (ii). Statement (iii) is a consequence of regularity results for the solutions of the Dirichlet problem, see [1].

Remark 2. It follows by Lemma 2.7 that if $\Omega$ is a bounded domain of class $C^{1,1}$ then $H(\Delta, \Omega) \cap H_{0}^{1}(\Omega)=$ $H^{2}(\Omega) \cap H_{0}^{1}(\Omega)$ and the two norms are equivalent. Indeed, it is well known that $C^{1,1}$ regularity implies the validity of the uniform outer ball condition.

\section{StRong SPECTRAL CONVERGENCE FOR SteKLOV AND STEKLOV-TyPe PROBlemS}

3.1. The functional setting. Assume that $\Omega \subset \mathbb{R}^{N}(N \geq 2)$ is a bounded domain of class $C^{0,1}$ satisfying the uniform exterior ball condition. To shorten our notation we set

$$
V(\Omega):=H^{2}(\Omega) \cap H_{0}^{1}(\Omega) .
$$

Proceeding as in [13], we relate the spectrum of the Steklov problem to the spectrum of a suitable selfadjoint compact operator acting on the space $V(\Omega)$. The space $V(\Omega)$ inherits the scalar product of $H^{2}(\Omega)$ and being closed in $H^{2}(\Omega)$ it becomes a Hilbert space. The space $V(\Omega)$ may be endowed with the two alternative scalar products

$$
\begin{aligned}
& (u, v) \mapsto \int_{\Omega} \Delta u \Delta v d x \quad \text { for any } u, v \in V(\Omega), \\
& (u, v) \mapsto \int_{\Omega} D^{2} u: D^{2} v d x \quad \text { for any } u, v \in V(\Omega) .
\end{aligned}
$$

Recall that by Lemma 2.7 (iii) the two scalar products defined in (15) and (16) are equivalent to the one induced by the $H^{2}(\Omega)$-norm. In the sequel we denote by $V^{\prime}(\Omega)$ the dual space of $V(\Omega)$.

Consider now the eigenvalue problem (2) and let us introduce a suitable characterization of its spectrum.

We introduce the operator $T_{\Delta}: V(\Omega) \mapsto V^{\prime}(\Omega)$ defined by

$$
V^{\prime}(\Omega)\left\langle T_{\Delta} u, v\right\rangle_{V(\Omega)}:=\int_{\Omega} \Delta u \Delta v d x \quad \text { for any } u, v \in V(\Omega) .
$$

The operator $T_{\Delta}$ is clearly well-defined and continuous. Moreover by Riesz Theorem we also deduce that $T_{\Delta}$ is invertible and $T_{\Delta}^{-1}$ is continuous. Then we introduce the operator $J: V(\Omega) \mapsto V^{\prime}(\Omega)$ defined by

$$
V^{\prime}(\Omega)\langle J u, v\rangle_{V(\Omega)}:=\int_{\partial \Omega} u_{\nu} v_{\nu} d S \quad \text { for any } u, v \in V(\Omega) .
$$

Since the linear map

$$
\begin{aligned}
V(\Omega) & \mapsto\left(L^{2}(\partial \Omega)\right)^{N} \\
u & \mapsto \nabla u_{\mid \partial \Omega}
\end{aligned}
$$

is well-defined and compact being $\partial \Omega$ Lipschitzian (see [31, Theorem 6.2, Chap. 2] for more details), then the operator $J$ is also well-defined and compact.

Next we introduce the operator $S_{\Delta}: V(\Omega) \mapsto V(\Omega)$ defined by $S_{\Delta}:=T_{\Delta}^{-1} \circ J$. Clearly $S_{\Delta}$ is a linear compact operator and moreover it is easy to see that it is also self-adjoint. Moreover one can show that $\mu \neq 0$ is an eigenvalue of $S_{\Delta}$ if and only if $d:=\mu^{-1}$ is an eigenvalue of (2). See the proof of Lemma 2 in [13] for more details.

With a completely similar procedure we can treat problem (5) by introducing the operator $T_{D^{2}}: V(\Omega) \rightarrow$ $V^{\prime}(\Omega)$ defined as

$$
V^{\prime}(\Omega)\left\langle T_{D^{2}} u, v\right\rangle_{V(\Omega)}:=\int_{\Omega} D^{2} u: D^{2} v d x \quad \text { for any } u, v \in V(\Omega) .
$$

Applying Riesz Theorem, we see that also the operator $T_{D^{2}}$ is invertible and its inverse function is continuous. Then one can define $S_{D^{2}}: V(\Omega) \rightarrow V(\Omega)$ as $S_{D^{2}}:=T_{D^{2}}^{-1} \circ J$ and prove that $S_{D^{2}}$ is a compact 
self-adjoint operator. Moreover any $\mu \neq 0$ is an eigenvalue of $S_{D^{2}}$ if and only if $\delta=\mu^{-1}$ is an eigenvalue of (5). This also proves the validity of (8) and show that the first eigenvalue $\delta_{1}$ admits the following characterization

$$
\delta_{1}=\inf _{v \in V(\Omega) \backslash H_{0}^{2}(\Omega)} \frac{\int_{\Omega}\left|D^{2} v\right|^{2} d x}{\int_{\partial \Omega} v_{\nu}^{2} d S} .
$$

3.2. Domain perturbations and definition of the operators $E_{\varepsilon}$. Let $\mathcal{A}$ be an atlas. We shall consider a family $\left\{\Omega_{\varepsilon}\right\}_{0<\varepsilon \leq \varepsilon_{0}}$ of domains of class $C^{1,1}(\mathcal{A})$ converging in a suitable sense to a fixed domain $\Omega$ of class $C^{1,1}(\mathcal{A})$. For any $0<\varepsilon \leq \varepsilon_{0}$ we introduce the Steklov operators

$$
S_{\Delta, \varepsilon}: V\left(\Omega_{\varepsilon}\right) \rightarrow V\left(\Omega_{\varepsilon}\right) \quad \text { and } \quad S_{D^{2}, \varepsilon}: V\left(\Omega_{\varepsilon}\right) \rightarrow V\left(\Omega_{\varepsilon}\right)
$$

according to the definition given in Section 3.1, and we study the convergence of $S_{\Delta, \varepsilon}$ to $S_{\Delta}$ and of $S_{D^{2}, \varepsilon}$ to $S_{D^{2}}$. To do so, we shall apply the general method described in Subsection 2.1 where the Hilbert spaces $\mathcal{H}_{\varepsilon}$ and $\mathcal{H}$ under consideration here are exactly $V\left(\Omega_{\varepsilon}\right)$ and $V(\Omega)$ endowed either with the scalar product (15) or with the scalar product (16).

In particular, we need to define a family of operators $E_{\varepsilon}$ as required in Subsection 2.1, which satisfy condition (11). In the specific case under consideration, this means that we have to introduce linear operators $E_{\varepsilon}: V(\Omega) \rightarrow V\left(\Omega_{\varepsilon}\right)$ such that

$$
\int_{\Omega_{\varepsilon}}\left|\Delta\left(E_{\varepsilon} u\right)\right|^{2} d x \rightarrow \int_{\Omega}|\Delta u|^{2} d x \quad \text { as } \varepsilon \rightarrow 0, \quad \text { for any } u \in V(\Omega)
$$

and

$$
\int_{\Omega_{\varepsilon}}\left|D^{2}\left(E_{\varepsilon} u\right)\right|^{2} d x \rightarrow \int_{\Omega}\left|D^{2} u\right|^{2} d x \quad \text { as } \varepsilon \rightarrow 0, \quad \text { for any } u \in V(\Omega) .
$$

To do so, we proceed as in [4]. Suppose that the following assumptions hold true: for any $\varepsilon \in\left(0, \varepsilon_{0}\right]$ there exists $\kappa_{\varepsilon}>0$ such that for any $j=1, \ldots, s^{\prime}$

$$
\left\|g_{\varepsilon, j}-g_{j}\right\|_{L^{\infty}\left(W_{j}\right)}<\kappa_{\varepsilon}, \quad \lim _{\varepsilon \rightarrow 0} \kappa_{\varepsilon}=0, \quad \lim _{\varepsilon \rightarrow 0} \frac{\left\|D^{\beta}\left(g_{\varepsilon, j}-g_{j}\right)\right\|_{L^{\infty}\left(W_{j}\right)}}{\kappa_{\varepsilon}^{3 / 2-|\beta|}}=0 \quad \forall \beta \in \mathbb{N}^{N} \text { with }|\beta| \leq 2 .
$$

Here we denoted by $g_{j}, g_{\varepsilon, j} \in C^{1,1}\left(\overline{W_{j}}\right)$ the functions corresponding respectively to $\Omega$ and $\Omega_{\varepsilon}$ according to Definition 2.6

Remark 3. One can prove that if

$$
D^{\alpha} g_{\varepsilon, j}\left(x^{\prime}\right) \rightarrow D^{\alpha} g_{j}\left(x^{\prime}\right) \text {, uniformly on } W_{j}
$$

for all $|\alpha| \leq 1$ and $j=1, \ldots, s^{\prime}$ and if there exists $M>0$ such that

$$
\left|D^{\alpha} g_{\varepsilon, j}\left(x^{\prime}\right)\right| \leq M \text {, a.e. on } W_{j}
$$

for all $|\alpha|=2$ and $j=1, \ldots, s^{\prime}$, then condition (21) is satisfied, see [4, Prop. 6.17]. We note that condition (21) is much less restrictive than conditions (22), [23), since it does not require the $L^{\infty}$-norms of the second order weak derivatives of functions $g_{\varepsilon, j}$ to be uniformly bounded. For example, in Corollary 4.1 if $3 / 2<$ $\alpha<2$ then condition (21) is satisfied but the $L^{\infty}$-norms of the second order weak derivatives of functions $g_{\varepsilon}$ are not uniformly bounded.

We are going to define the operator $E_{\varepsilon}$ by using a partition of unity and pasting together suitable pull-back operators associated with local diffeomorphisms defined on each cuboid of the atlas $\mathcal{A}$. Note that in the simplified setting of one single cuboid, partition of unity would not be required and the operator $E_{\varepsilon}$ would be simply defined as in Remark 4 below.

We denote by $\hat{k}$ a fixed constant satisfying $\hat{k}>6$ whose meaning will be clear below. We also put $k_{\varepsilon}:=\hat{k} \kappa_{\varepsilon}, \tilde{g}_{\varepsilon, j}:=g_{\varepsilon, j}-k_{\varepsilon}$ and $K_{\varepsilon, j}:=\left\{\left(x^{\prime}, x_{N}\right) \in W_{j} \times\left(a_{N j}, b_{N j}\right): a_{N j}<x_{N}<\tilde{g}_{\varepsilon, j}\left(x^{\prime}\right)\right\}$ for any $j=1, \ldots, s^{\prime}$. 
For any $1 \leq j \leq s^{\prime}$ we define the map $h_{\varepsilon, j}: r_{j}\left(\overline{\Omega_{\varepsilon} \cap V_{j}}\right) \rightarrow \mathbb{R}$

$$
h_{\varepsilon, j}\left(x^{\prime}, x_{N}\right):= \begin{cases}0, & \text { if } a_{j N} \leq x_{N} \leq \tilde{g}_{\varepsilon, j}\left(x^{\prime}\right), \\ \left(g_{\varepsilon, j}\left(x^{\prime}\right)-g_{j}\left(x^{\prime}\right)\right)\left(\frac{x_{N}-\tilde{g}_{\varepsilon, j}\left(x^{\prime}\right)}{g_{\varepsilon, j}\left(x^{\prime}\right)-\tilde{g}_{\varepsilon, j}\left(x^{\prime}\right)}\right)^{3}, & \text { if } \tilde{g}_{\varepsilon, j}\left(x^{\prime}\right)<x_{N} \leq g_{\varepsilon, j}\left(x^{\prime}\right) .\end{cases}
$$

We observe that $h_{\varepsilon, j} \in C^{1,1}\left(r_{j}\left(\overline{\Omega_{\varepsilon} \cap V_{j}}\right)\right)$ and that the map $\Phi_{\varepsilon, j}: r_{j}\left(\overline{\Omega_{\varepsilon} \cap V_{j}}\right) \rightarrow r_{j}\left(\overline{\Omega \cap V_{j}}\right)$ defined by $\Phi_{\varepsilon, j}\left(x^{\prime}, x_{N}\right):=\left(x^{\prime}, x_{N}-h_{\varepsilon, j}\left(x^{\prime}, x_{N}\right)\right)$ is a diffeomorphism of class $C^{1,1}$. When $s^{\prime}+1 \leq j \leq s$ we define $\Phi_{\varepsilon, j}: r_{j}\left(\overline{V_{j}}\right) \rightarrow r_{j}\left(\overline{V_{j}}\right)$ as the identity map.

Exploiting the definition of $\Phi_{\varepsilon, j}$ we may define the above mentioned linear map $E_{\varepsilon}$. Consider a partition of unity $\left\{\psi_{j}\right\}_{1 \leq j \leq s}$ subordinate to the open cover $\left\{V_{j}\right\}_{1 \leq j \leq s}$ of the compact set $\overline{\Omega \cup \bigcup_{\varepsilon \in\left(0, \varepsilon_{0}\right]} \Omega_{\varepsilon}}$, i.e., a set of functions $\left\{\psi_{j}\right\}_{1 \leq j \leq s}$ such that $\operatorname{supp}\left(\psi_{j}\right) \subset V_{j}$ for any $j \in\{1, \ldots, s\}$ and $\sum_{j=1}^{s} \psi_{j} \equiv 1$ in $\overline{\Omega \cup \bigcup_{\varepsilon \in\left(0, \varepsilon_{0}\right]} \Omega_{\varepsilon}}$. Indeed one can apply for example [11, Lemma IX.3] with $\Gamma=\overline{\Omega \cup \bigcup_{\varepsilon \in\left(0, \varepsilon_{0}\right]} \Omega_{\varepsilon}}$. Note that this partition of unity is independent of $\varepsilon$. We also define the deformation $\Psi_{\varepsilon, j}: \overline{\Omega_{\varepsilon} \cap V_{j}} \rightarrow \overline{\Omega \cap V_{j}}$ by $\Psi_{\varepsilon, j}:=r_{j}^{-1} \circ \Phi_{\varepsilon, j} \circ r_{j}$. In this way $\Psi_{\varepsilon, j}$ becomes a $C^{1,1}$ diffeomorphism from $\overline{\Omega_{\varepsilon} \cap V_{j}}$ onto $\overline{\Omega \cap V_{j}}$ for any $j \in\{1, \ldots, s\}$.

From the definition of $h_{\varepsilon, j}$ and the restriction $\hat{k}>6$, we deduce that

$$
\frac{1}{2} \leq \operatorname{det}\left(D \Psi_{\varepsilon, j}(x)\right) \leq \frac{3}{2} \quad \text { for any } x \in \Omega_{\varepsilon} \cap V_{j} .
$$

In order to show this, we observe that $\operatorname{det}\left(D \Psi_{\varepsilon, j}\right)=\operatorname{det}\left(D \Phi_{\varepsilon, j}\right)=1-\frac{\partial h_{\varepsilon, j}}{\partial x_{N}}\left(x^{\prime}, x_{N}\right)$.

Given $u \in V(\Omega)$ we put $u_{j}=\psi_{j} u$ for any $j \in\{1, \ldots, s\}$ in such a way that $u=\sum_{j=1}^{s} u_{j}$. Then we define

$$
E_{\varepsilon} u:=\sum_{j=1}^{s^{\prime}} \tilde{u}_{\varepsilon, j}+\sum_{j=s^{\prime}+1}^{s} u_{j} \in V\left(\Omega_{\varepsilon}\right)
$$

where

$$
\tilde{u}_{\varepsilon, j}(x)= \begin{cases}u_{j}\left(\Psi_{\varepsilon, j}(x)\right) & \text { if } x \in \Omega_{\varepsilon} \cap V_{j} \\ 0 & \text { if } x \in \Omega_{\varepsilon} \backslash V_{j}\end{cases}
$$

for any $j \in\left\{1, \ldots, s^{\prime}\right\}$.

Remark 4. If $\Omega$ and $\Omega_{\varepsilon}$ are in the form

$$
\begin{aligned}
& \Omega=\left\{\left(x^{\prime}, x_{N}\right) \in \mathbb{R}^{N}: x^{\prime} \in W \text { and } a_{N}<x_{N}<g\left(x^{\prime}\right)\right\}, \\
& \Omega_{\varepsilon}=\left\{\left(x^{\prime}, x_{N}\right) \in \mathbb{R}^{N}: x^{\prime} \in W \text { and } a_{N}<x_{N}<g_{\varepsilon}\left(x^{\prime}\right)\right\},
\end{aligned}
$$

where $W$ is a cuboid or a bounded domain in $\mathbb{R}^{N-1}$ of class $C^{1,1}$ then the operator $E_{\varepsilon}$ can be defined in the following simple way

$$
E_{\varepsilon} u(x)=u\left(\Phi_{\varepsilon}(x)\right) \quad \text { for any } x \in \Omega_{\varepsilon}
$$

where $\Phi_{\varepsilon}\left(x^{\prime}, x_{N}\right)=\left(x^{\prime}, x_{N}-h_{\varepsilon}\left(x^{\prime}, x_{N}\right)\right)$ and $h_{\varepsilon}$ is defined by (24) with $g_{\varepsilon}$ and $g$ in place of $g_{\varepsilon, j}$ and $g_{j}$ respectively.

Lemma 3.1. Let $\mathcal{A}$ be an atlas. Let $\left\{\Omega_{\varepsilon}\right\}_{0<\varepsilon \leq \varepsilon_{0}}$ be a family of domains of class $C^{1,1}(\mathcal{A}), \Omega$ a domain of class $C^{1,1}(\mathcal{A})$ and for any $\varepsilon \in\left(0, \varepsilon_{0}\right]$ let $E_{\varepsilon}$ be the map defined in (26). Assume the validity of condition (21).

Then the following assertions hold true:

(i) the map $E_{\varepsilon}: V(\Omega) \rightarrow V\left(\Omega_{\varepsilon}\right)$ is continuous for any $\varepsilon \in\left(0, \varepsilon_{0}\right]$;

(ii) the family of operators $\left\{E_{\varepsilon}\right\}_{0<\varepsilon \leq \varepsilon_{0}}$ satisfies (19) and (20).

Proof. Since the proof of (i) easily follows from the definition of $E_{\varepsilon}$, it is left to the reader. 
We now prove (19). Let $u \in V(\Omega)$ and according to (27) introduce the functions $\tilde{u}_{\varepsilon, j}$. Throughout the proof of this lemma we denote by $C$ a positive constant independent of $\varepsilon$ which may vary from line to line. We observe that in order to prove (19), it is sufficient to show that

$$
\lim _{\varepsilon \rightarrow 0} \int_{\Omega_{\varepsilon}} \frac{\partial^{2} \tilde{u}_{\varepsilon, i}}{\partial x_{k}^{2}} \frac{\partial^{2} \tilde{u}_{\varepsilon, j}}{\partial x_{l}^{2}} d x=\int_{\Omega} \frac{\partial^{2} u_{i}}{\partial x_{k}^{2}} \frac{\partial^{2} u_{j}}{\partial x_{l}^{2}} d x
$$

for any $i, j \in\left\{1, \ldots, s^{\prime}\right\}$ and $k, l \in\{1, \ldots, N\}$. We first prove that (28) holds when $i=j$ and $k=l$. One can proceed similarly to the proof of [4, Lemma 6.2] and hence we only give here a sketch of it. By (21) and (6.7) in [4] we have that

$$
\left\|D^{\alpha} h_{\varepsilon, j}\right\|_{L^{\infty}\left(W_{j}\right)} \leq C \sum_{0 \leq \gamma \leq \alpha} \frac{\left\|D^{\gamma}\left(g_{\varepsilon, j}-g_{j}\right)\right\|_{L^{\infty}\left(W_{j}\right)}}{\kappa_{\varepsilon}^{|\alpha|-|\gamma|}}
$$

for any $\alpha \in \mathbb{N}^{N}$ with $|\alpha| \leq 2$. By $\gamma \leq \alpha$ we mean that the inequality holds component by component. For any $x \in \Omega_{\varepsilon} \cap V_{i}$ we have

$$
\frac{\partial^{2} \tilde{u}_{\varepsilon, i}}{\partial x_{k}^{2}}(x)=\sum_{j=1}^{N}\left\{\left[\sum_{l=1}^{N} \frac{\partial^{2} u_{i}}{\partial x_{l} \partial x_{j}}\left(\Psi_{\varepsilon, i}(x)\right) \frac{\partial\left[\left(\Psi_{\varepsilon, i}(x)\right)_{l}\right]}{\partial x_{k}}\right] \frac{\partial\left[\left(\Psi_{\varepsilon, i}(x)\right)_{j}\right]}{\partial x_{k}}+\frac{\partial u_{i}}{\partial x_{j}}\left(\Psi_{\varepsilon, i}(x)\right) \frac{\partial^{2}\left[\left(\Psi_{\varepsilon, i}(x)\right)_{j}\right]}{\partial x_{k}^{2}}\right\} .
$$

By (21), 29) and the definitions of $\Phi_{\varepsilon, i}, \Psi_{\varepsilon, i}$, we deduce that there exists $C>0$ independent of $\varepsilon$ such that (31)

$$
\left\|\frac{\partial\left[\left(\Psi_{\varepsilon, i}(x)\right)_{l}\right]}{\partial x_{k}} \frac{\partial\left[\left(\Psi_{\varepsilon, i}(x)\right)_{j}\right]}{\partial x_{k}}\right\|_{L^{\infty}\left(V_{i} \cap \Omega_{\varepsilon}\right)} \leq C \quad \text { and } \quad\left\|\frac{\partial^{2}\left[\left(\Psi_{\varepsilon, i}(x)\right)_{j}\right]}{\partial x_{k}^{2}}\right\|_{L^{\infty}\left(V_{i} \cap \Omega_{\varepsilon}\right)}=o\left(\kappa_{\varepsilon}^{-1 / 2}\right) \text { as } \varepsilon \rightarrow 0 .
$$

Exploiting (30)-(31) and proceeding as in the proof of [4, Lemma 6.2] we deduce that

$$
\lim _{\varepsilon \rightarrow 0} \int_{\left(\Omega_{\varepsilon} \cap V_{i}\right) \backslash r_{i}^{-1}\left(K_{\varepsilon, i}\right)}\left(\frac{\partial^{2} \tilde{u}_{\varepsilon, i}}{\partial x_{k}^{2}}\right)^{2} d x=0 .
$$

We briefly give a proof of (32). First of all by (25) we have

$$
\int_{\left(\Omega_{\varepsilon} \cap V_{i}\right) \backslash r_{i}^{-1}\left(K_{\varepsilon, i}\right)}\left(\frac{\partial^{2} u_{i}}{\partial x_{l} \partial x_{j}}\left(\Psi_{\varepsilon, i}(x)\right)\right)^{2} d x \leq C \int_{\left(\Omega \cap V_{i}\right) \backslash r_{i}^{-1}\left(K_{\varepsilon, i}\right)}\left(\frac{\partial^{2} u_{i}}{\partial x_{l} \partial x_{j}}\right)^{2} d x \rightarrow 0
$$

as $\varepsilon \rightarrow 0$ since $\left|\left(\Omega \cap V_{i}\right) \backslash r_{i}^{-1}\left(K_{\varepsilon, i}\right)\right| \rightarrow 0$ as $\varepsilon \rightarrow 0$.

On the other hand, putting $v_{i}(z)=u_{i}\left(r_{i}^{-1}(z)\right)$ for any $z \in r_{i}\left(\Omega \cap V_{i}\right)$, by (21), (31) and changes of variables, we have

$$
\begin{aligned}
& \int_{\left(\Omega_{\varepsilon} \cap V_{i}\right) \backslash r_{i}^{-1}\left(K_{\varepsilon, i}\right)}\left(\frac{\partial u_{i}}{\partial x_{j}}\left(\Psi_{\varepsilon, i}(x)\right) \frac{\partial^{2}\left[\left(\Psi_{\varepsilon, i}(x)\right)_{j}\right]}{\partial x_{k}^{2}}\right)^{2} d x \leq o\left(\kappa_{\varepsilon}^{-1}\right) \sum_{l=1}^{N} \int_{r_{i}\left(\Omega \cap V_{i}\right) \backslash K_{\varepsilon, i}}\left(\frac{\partial v_{i}}{\partial z_{l}}\right)^{2} d z \\
& =o\left(\kappa_{\varepsilon}^{-1}\right) \sum_{l=1}^{N} \int_{W_{i}}\left(\int_{\tilde{g}_{\varepsilon, i}\left(z^{\prime}\right)}^{g_{i}\left(z^{\prime}\right)}\left(\frac{\partial v_{i}}{\partial z_{l}}\left(z^{\prime}, z_{N}\right)\right)^{2} d z_{N}\right) d z^{\prime} \\
& \leq o\left(\kappa_{\varepsilon}^{-1}\right) \sum_{l=1}^{N} \int_{W_{i}}\left|g_{i}\left(z^{\prime}\right)-\tilde{g}_{\varepsilon, i}\left(z^{\prime}\right)\right|\left\|\frac{\partial v_{i}}{\partial z_{l}}\left(z^{\prime}, \cdot\right)\right\|_{L^{\infty}\left(a_{N i}, g_{i}\left(z^{\prime}\right)\right)}^{2} d z^{\prime} \\
& \leq o(1) \sum_{l=1}^{N} \int_{W_{i}}\left\|\frac{\partial v_{i}}{\partial z_{l}}\left(z^{\prime}, \cdot\right)\right\|_{H^{1}\left(a_{N i}, g_{i}\left(z^{\prime}\right)\right)}^{2} d z^{\prime} \\
& \leq o(1)\left\|v_{i}\right\|_{H^{2}\left(r_{i}\left(\Omega \cap V_{i}\right)\right)}=o(1) .
\end{aligned}
$$

Note that here we have used the classical one dimensional estimate

$$
\|f\|_{L^{\infty}(a, b)} \leq C\|f\|_{H^{1}(a, b)}
$$


for all $f \in H^{1}(a, b)$ with a constant $C=C(d)$ uniformly bounded for $b-a>d$. Combining (33), (34) with (30) and (31), (32) follows.

On the other hand

$$
\lim _{\varepsilon \rightarrow 0} \int_{r_{i}^{-1}\left(K_{\varepsilon, i}\right)}\left(\frac{\partial^{2} \tilde{u}_{\varepsilon, i}}{\partial x_{k}^{2}}\right)^{2} d x=\lim _{\varepsilon \rightarrow 0} \int_{r_{i}^{-1}\left(K_{\varepsilon, i}\right)}\left(\frac{\partial^{2} u_{i}}{\partial x_{k}^{2}}\right)^{2} d x=\int_{\Omega \cap V_{i}}\left(\frac{\partial^{2} u_{i}}{\partial x_{k}^{2}}\right)^{2} d x=\int_{\Omega}\left(\frac{\partial^{2} u_{i}}{\partial x_{k}^{2}}\right)^{2} d x .
$$

Combining (32)-(36) we conclude the proof of (28) in the case $i=j$ and $k=l$.

It remains to prove (28) in the general case.

We observe that

$$
\begin{aligned}
& \int_{\Omega_{\varepsilon}} \frac{\partial^{2} \tilde{u}_{\varepsilon, i}}{\partial x_{k}^{2}} \frac{\partial^{2} \tilde{u}_{\varepsilon, j}}{\partial x_{l}^{2}} d x=\int_{\Omega_{\varepsilon} \cap V_{i} \cap V_{j}} \frac{\partial^{2} \tilde{u}_{\varepsilon, i}}{\partial x_{k}^{2}} \frac{\partial^{2} \tilde{u}_{\varepsilon, j}}{\partial x_{l}^{2}} d x \\
& =\int_{r_{i}^{-1}\left(K_{\varepsilon, i}\right) \cap r_{j}^{-1}\left(K_{\varepsilon, j}\right)} \frac{\partial^{2} \tilde{u}_{\varepsilon, i}}{\partial x_{k}^{2}} \frac{\partial^{2} \tilde{u}_{\varepsilon, j}}{\partial x_{l}^{2}} d x+\int_{\left(\Omega_{\varepsilon} \cap V_{i} \cap V_{j}\right) \backslash\left(r_{i}^{-1}\left(K_{\varepsilon, i}\right) \cap r_{j}^{-1}\left(K_{\varepsilon, j}\right)\right)} \frac{\partial^{2} \tilde{u}_{\varepsilon, i}}{\partial x_{k}^{2}} \frac{\partial^{2} \tilde{u}_{\varepsilon, j}}{\partial x_{l}^{2}} d x .
\end{aligned}
$$

We have that $\Psi_{\varepsilon, i}$ and $\Psi_{\varepsilon, j}$ are the identity maps if restricted to $r_{i}^{-1}\left(K_{\varepsilon, i}\right) \cap r_{j}^{-1}\left(K_{\varepsilon, j}\right)$ so that by (21) we obtain

$$
\lim _{\varepsilon \rightarrow 0} \int_{r_{i}^{-1}\left(K_{\varepsilon, i}\right) \cap r_{j}^{-1}\left(K_{\varepsilon, j}\right)} \frac{\partial^{2} \tilde{u}_{\varepsilon, i}}{\partial x_{k}^{2}} \frac{\partial^{2} \tilde{u}_{\varepsilon, j}}{\partial x_{l}^{2}} d x=\lim _{\varepsilon \rightarrow 0} \int_{r_{i}^{-1}\left(K_{\varepsilon, i}\right) \cap r_{j}^{-1}\left(K_{\varepsilon, j}\right)} \frac{\partial^{2} u_{i}}{\partial x_{k}^{2}} \frac{\partial^{2} u_{j}}{\partial x_{l}^{2}} d x=\int_{\Omega} \frac{\partial^{2} u_{i}}{\partial x_{k}^{2}} \frac{\partial^{2} u_{j}}{\partial x_{l}^{2}} d x .
$$

In order to estimate the other term in the right hand side of (37), we split the domain of integration in the following way

$$
\left(\Omega_{\varepsilon} \cap V_{i} \cap V_{j}\right) \backslash\left(r_{i}^{-1}\left(K_{\varepsilon, i}\right) \cap r_{j}^{-1}\left(K_{\varepsilon, j}\right)\right)=\left[\left(\Omega_{\varepsilon} \cap V_{i} \cap V_{j}\right) \backslash r_{i}^{-1}\left(K_{\varepsilon, i}\right)\right] \cup\left[\left(r_{i}^{-1}\left(K_{\varepsilon, i}\right) \backslash r_{j}^{-1}\left(K_{\varepsilon, j}\right)\right) \cap V_{j}\right] .
$$

Then

$$
\begin{aligned}
& \left|\int_{\left(\Omega_{\varepsilon} \cap V_{i} \cap V_{j}\right) \backslash r_{i}^{-1}\left(K_{\varepsilon, i}\right)} \frac{\partial^{2} \tilde{u}_{\varepsilon, i}}{\partial x_{k}^{2}} \frac{\partial^{2} \tilde{u}_{\varepsilon, j}}{\partial x_{l}^{2}} d x\right| \\
& \leq\left(\int_{\left(\Omega_{\varepsilon} \cap V_{i}\right) \backslash r_{i}^{-1}\left(K_{\varepsilon, i}\right)}\left(\frac{\partial^{2} \tilde{u}_{\varepsilon, i}}{\partial x_{k}^{2}}\right)^{2} d x\right)^{1 / 2}\left(\int_{\Omega_{\varepsilon} \cap V_{j}}\left(\frac{\partial^{2} \tilde{u}_{\varepsilon, j}}{\partial x_{l}^{2}}\right)^{2} d x\right)^{1 / 2} \rightarrow 0 \quad \text { as } \varepsilon \rightarrow 0
\end{aligned}
$$

in view of (32) and (28) in the case $i=j$ and $k=l$. On the other hand

$$
\begin{aligned}
& \left|\int_{\left(r_{i}^{-1}\left(K_{\varepsilon, i}\right) \backslash r_{j}^{-1}\left(K_{\varepsilon, j}\right)\right) \cap V_{j}} \frac{\partial^{2} \tilde{u}_{\varepsilon, i}}{\partial x_{k}^{2}} \frac{\partial^{2} \tilde{u}_{\varepsilon, j}}{\partial x_{l}^{2}} d x\right| \\
& \leq\left(\int_{r_{i}^{-1}\left(K_{\varepsilon, i}\right)}\left(\frac{\partial^{2} \tilde{u}_{\varepsilon, i}}{\partial x_{k}^{2}}\right)^{2} d x\right)^{1 / 2}\left(\int_{\left(\Omega_{\varepsilon} \cap V_{j}\right) \backslash r_{j}^{-1}\left(K_{\varepsilon, j}\right)}\left(\frac{\partial^{2} \tilde{u}_{\varepsilon, j}}{\partial x_{l}^{2}}\right)^{2} d x\right)^{1 / 2} \rightarrow 0 \quad \text { as } \varepsilon \rightarrow 0
\end{aligned}
$$

in view of (32) and (36).

Combining (38)-(41) with (37), the proof of (28) follows also in the general case.

We now prove the validity of (20). We observe that it is sufficient to show that

$$
\lim _{\varepsilon \rightarrow 0} \int_{\Omega_{\varepsilon}} \frac{\partial^{2} \tilde{u}_{\varepsilon, i}}{\partial x_{k} \partial x_{l}} \frac{\partial^{2} \tilde{u}_{\varepsilon, j}}{\partial x_{k} \partial x_{l}} d x=\int_{\Omega} \frac{\partial^{2} u_{i}}{\partial x_{k} \partial x_{l}} \frac{\partial^{2} u_{j}}{\partial x_{k} \partial x_{l}} d x .
$$

The procedure that one has to follow to prove (42) is essentially the same adopted in Step 2: first one has to prove (42) in the case $i=j$ with $k, l \in\{1, \ldots, N\}$ not necessarily equal and then one has to pass to the general case by arguing as above. 
3.3. The stability result. In this subsection we prove the following result on spectral convergence for problems (2) and (5). One of the main assumptions is condition (21) which, as we can see from Lemma 3.1 , guarantees the validity of the properties (19) and (20).

Theorem 3.2. Let $\mathcal{A}$ be an atlas. Let $\left\{\Omega_{\varepsilon}\right\}_{0<\varepsilon \leq \varepsilon_{0}}$ be a family of domains of class $C^{1,1}(\mathcal{A})$ and let $\Omega$ be a domain of class $C^{1,1}(\mathcal{A})$. Assume the validity of condition (21). Then $S_{\Delta, \varepsilon} \stackrel{C}{\longrightarrow} S_{\Delta}$ and $S_{D^{2}, \varepsilon} \stackrel{C}{\longrightarrow} S_{D^{2}}$ with respect to the operators $E_{\varepsilon}$ defined in (26). In particular, the spectra of (2) and (5) behave continuously at $\varepsilon=0$ in the sense of Theorem 2.5

Proof. The proof of Theorem 3.2 follows directly by Theorem 2.5 and Lemmas 3.93 3.10 below.

Remark 5. In the case of a single cuboid we can state a simplified version of Theorem 3.2 the proof of which would follow the same lines of the proof of Theorem 3.2 with obvious modifications. Namely, if $\Omega, \Omega_{\varepsilon}$ are as in Remark 4 with $g_{\varepsilon}$ and $g$ satisfying (21) with $g_{\varepsilon}$ and $g$ in place of $g_{\varepsilon, j}$ and $g_{j}$ respectively, then $S_{\Delta, \varepsilon} \stackrel{C}{\longrightarrow} S_{\Delta}$ and $S_{D^{2}, \varepsilon} \stackrel{C}{\longrightarrow} S_{D^{2}}$ with respect to the operators $E_{\varepsilon}$ defined in Remark 4 In particular, the spectra of (2) and (5) behave continuously at $\varepsilon=0$ in the sense of Theorem 2.5

Note that although in this specific case $\Omega_{\varepsilon}$ and $\Omega$ are not of class $C^{1,1}$ but only piecewise $C^{1,1}$, each of them is of class $C^{0,1}$ and satisfies the uniform outer ball condition.

In order to prove Lemma 3.9 and Lemma 3.10 , we need a number of preliminary technical results.

To begin with, we give the definition of a map which acts between the spaces $V(\Omega), V\left(\Omega_{\varepsilon}\right)$ in a reversed way with respect to $E_{\varepsilon}$. For any $w \in V\left(\Omega_{\varepsilon}\right)$ put

$$
\widehat{w}_{\varepsilon, j}(x)= \begin{cases}w_{j}\left(\Psi_{\varepsilon, j}^{-1}(x)\right), & \text { if } x \in \Omega \cap V_{j} \\ 0, & \text { if } x \in \Omega \backslash V_{j} .\end{cases}
$$

for any $j \in\left\{1, \ldots, s^{\prime}\right\}$ and $w_{j}:=\psi_{j} w$ for any $j \in\{1, \ldots, s\}$. We define

$$
B_{\varepsilon} w:=\sum_{j=1}^{s^{\prime}} \widehat{w}_{\varepsilon, j}+\sum_{j=s^{\prime}+1}^{s} w_{j} .
$$

In this way we have constructed a map $B_{\varepsilon}: V\left(\Omega_{\varepsilon}\right) \rightarrow V(\Omega)$.

Lemma 3.3. Let $\mathcal{A}$ be an atlas. Let $\left\{\Omega_{\varepsilon}\right\}_{0<\varepsilon \leq \varepsilon_{0}}$ be a family of domains of class $C^{1,1}(\mathcal{A})$ and $\Omega$ a domain of class $C^{1,1}(\mathcal{A})$. Assume the validity of condition (21). Then, up to shrink $\varepsilon_{0}$ if necessary,

$$
\left\|B_{\varepsilon} w\right\|_{H^{2}(\Omega)} \leq C\|\Delta w\|_{L^{2}\left(\Omega_{\varepsilon}\right)} \quad \text { for any } w \in V\left(\Omega_{\varepsilon}\right) \text { and } \varepsilon \in\left(0, \varepsilon_{0}\right]
$$

where $C>0$ is a constant independent of $\varepsilon$.

Proof. Throughout the proof we denote by $C$ a general constant independent of $\varepsilon$ which may vary from line to line. Let $w \in V\left(\Omega_{\varepsilon}\right)$. Since by (21) $\left|\Omega_{\varepsilon}\right| \rightarrow|\Omega|$ as $\varepsilon \rightarrow 0$ and the Poincaré constant depends only on the dimension of the space and the volume of the domain (see the comment before Lemma 2.7), we deduce by (14) that possibly shrinking $\varepsilon_{0}$

$$
\int_{\Omega_{\varepsilon}}|\nabla w|^{2} d x \leq C\|\Delta w\|_{L^{2}\left(\Omega_{\varepsilon}\right)}^{2} \text { and } \int_{\Omega_{\varepsilon}} w^{2} d x \leq C\|\Delta w\|_{L^{2}\left(\Omega_{\varepsilon}\right)}^{2}
$$

for all $\varepsilon \in\left(0, \varepsilon_{0}\right]$. Define $w_{j}:=\psi_{j} w$. Then by (45) and the fact that $\Delta w_{j}=w \Delta \psi_{j}+2 \nabla \psi_{j} \cdot \nabla w+\psi_{j} \Delta w$, it follows

$$
\left\|\Delta w_{j}\right\|_{L^{2}\left(\Omega_{\varepsilon}\right)} \leq C\|\Delta w\|_{L^{2}\left(\Omega_{\varepsilon}\right)} .
$$

In the rest of the proof we fix $j \in\left\{1, \ldots, s^{\prime}\right\}$. By direct computation one can show that the function $\widehat{w}_{\varepsilon, j}$ defined in (43) satisfies

$$
\operatorname{div}\left(A_{\varepsilon}(y) \nabla \widehat{w}_{\varepsilon, j}(y)\right)=\left[\operatorname{det}\left(D \Psi_{\varepsilon, j}\left(\Psi_{\varepsilon, j}^{-1}(y)\right)\right)\right]^{-1} \Delta w_{j}\left(\Psi_{\varepsilon, j}^{-1}(y)\right) \quad \text { for any } y \in \Omega \cap V_{j}
$$


where $A_{\varepsilon}(y):=\left[\operatorname{det}\left(D \Psi_{\varepsilon, j}\left(\Psi_{\varepsilon, j}^{-1}(y)\right)\right)\right]^{-1}\left[D \Psi_{\varepsilon, j}\left(\Psi_{\varepsilon, j}^{-1}(y)\right)\right]\left[D \Psi_{\varepsilon, j}\left(\Psi_{\varepsilon, j}^{-1}(y)\right)\right]^{T}$. We may think to the function $\Phi_{\varepsilon, j}$ as defined over all the set $\left\{\left(x^{\prime}, x_{N}\right) \in \mathbb{R}^{N}: x^{\prime} \in W_{j}, x_{N} \leq g_{\varepsilon, j}\left(x^{\prime}\right)\right\}$ since we may extend trivially the function $h_{\varepsilon, j}$ over the same set. Let us introduce the operator $L_{\varepsilon} v:=\operatorname{div}\left(A_{\varepsilon}(y) \nabla v\right)$ for any function $v \in V(\Omega)$. Then we have

$$
\int_{\Omega \cap V_{j}}\left|L_{\varepsilon} \widehat{w}_{\varepsilon, j}\right|^{2} d y=\int_{\Omega_{\varepsilon} \cap V_{j}}\left|\Delta w_{j}\right|^{2}\left[\operatorname{det}\left(D \Psi_{\varepsilon, j}\right)\right]^{-1} d x
$$

Combining (46), (47) and (25), we deduce that

$$
\int_{\Omega \cap V_{j}}\left|L_{\varepsilon} \widehat{w}_{\varepsilon, j}\right|^{2} d y \leq C\left\|\Delta w_{j}\right\|_{L^{2}\left(\Omega_{\varepsilon}\right)}^{2} \leq C\|\Delta w\|_{L^{2}\left(\Omega_{\varepsilon}\right)}^{2} \quad \text { for any } \varepsilon \in\left(0, \varepsilon_{0}\right] .
$$

In order to complete the proof of the lemma we prove the following claim: there exists a constant $C$ independent of $\varepsilon$ such that

$$
\|u\|_{H^{2}(\Omega)} \leq C\left\|L_{\varepsilon} u\right\|_{L^{2}\left(\Omega \cap V_{j}\right)} \quad \text { for any } u \in V(\Omega), \operatorname{supp}(u) \subset V_{j}, \text { and } \varepsilon \in\left(0, \varepsilon_{0}\right] .
$$

In order to do that, we proceed by using a standard method from the theory of regularity.

For any $u \in V(\Omega)$ with $\operatorname{supp}(u) \subset V_{j}$, we define the function $f_{\varepsilon} \in L^{2}\left(r_{j}^{-1}\left(\Sigma_{j}\right)\right)$ by setting $f_{\varepsilon}(y)=$ $-L_{\varepsilon} u(y)$ if $y \in \Omega \cap V_{j}$ and $f_{\varepsilon}(y)=0$ if $y \in r_{j}^{-1}\left(\Sigma_{j}\right) \backslash\left(\Omega \cap V_{j}\right)$ where

$$
\Sigma_{j}:=\left\{\left(x^{\prime}, x_{N}\right) \in \mathbb{R}^{N}: x^{\prime} \in W_{j}, x_{N} \leq g_{j}\left(x^{\prime}\right)\right\} .
$$

We define the deformation

$$
\begin{aligned}
& \widetilde{\Phi}_{j}: \Sigma_{j} \rightarrow W_{j} \times(-\infty, 0] \\
& \widetilde{\Phi}_{j}\left(x^{\prime}, x_{N}\right):=\left(x^{\prime}, x_{N}-g_{j}\left(x^{\prime}\right)\right)
\end{aligned}
$$

and the function $\tilde{u}: W_{j} \times(-\infty, 0] \rightarrow \mathbb{R}$ which is defined by $\tilde{u}(y)=u\left(r_{j}^{-1}\left(\widetilde{\Phi}_{j}^{-1}(y)\right)\right)$ if $y \in \widetilde{\Phi}_{j}\left(r_{j}\left(\Omega \cap V_{j}\right)\right)$ and $\tilde{u}(y)=0$ if $y \in\left(W_{j} \times(-\infty, 0)\right) \backslash \widetilde{\Phi}_{j}\left(r_{j}\left(\Omega \cap V_{j}\right)\right)$.

We observe that $\tilde{u} \in H^{2}\left(W_{j} \times(-\infty, 0)\right) \cap H_{0}^{1}\left(W_{j} \times(-\infty, 0)\right)$ and its support is a compact set contained in $W_{j} \times(-\infty, 0]$ thus allowing to extend it trivially over all $\mathbb{R}^{N-1} \times(-\infty, 0)$; we still denote by $\tilde{u}$ this trivial extension. Moreover, we set $\widetilde{\Psi}_{j}(z):=\widetilde{\Phi}_{j}\left(r_{j}(z)\right)$ for all $z \in r_{j}^{-1}\left(\Sigma_{j}\right)$, and we note that $\tilde{u}$ solves the equation

$$
-\operatorname{div}\left(\tilde{A}_{\varepsilon}(y) \nabla \tilde{u}\right)=\tilde{f}_{\varepsilon} \quad \text { in } W_{j} \times(-\infty, 0)
$$

where

$$
\tilde{A}_{\varepsilon}(y):=\left[\operatorname{det}\left(D \widetilde{\Psi}_{j}\left(\widetilde{\Psi}_{j}^{-1}(y)\right)\right)\right]^{-1}\left[D \widetilde{\Psi}_{j}\left(\widetilde{\Psi}_{j}^{-1}(y)\right)\right] A_{\varepsilon}\left(\widetilde{\Psi}_{j}^{-1}(y)\right)\left[D \widetilde{\Psi}_{j}\left(\widetilde{\Psi}_{j}^{-1}(y)\right)\right]^{T}
$$

and $\tilde{f}_{\varepsilon}(y):=\left[\operatorname{det}\left(D \widetilde{\Psi}_{j}\left(\widetilde{\Psi}_{j}^{-1}(y)\right)\right)\right]^{-1} f_{\varepsilon}\left(\widetilde{\Psi}_{j}^{-1}(y)\right)$ for any $y \in W_{j} \times(-\infty, 0)$.

We point out that, in the definition of $\tilde{A}_{\varepsilon}$, the function $A_{\varepsilon}$ has been extended by zero over the set $r_{j}^{-1}\left(\Sigma_{j}\right)$.

Then, we perform the Nirenberg translation method. In order to estimate second derivatives of $\tilde{u}$, we fix arbitrarily $i \in\{1, \ldots, N-1\}$ and we define $\tilde{u}_{h}(x):=\tilde{u}\left(x+h e_{i}\right)$ for any $h \in \mathbb{R}$ where we have omitted for simplicity the index $i$. We use the same notation for any function $v \in H_{0}^{1}\left(\mathbb{R}^{N-1} \times(-\infty, 0)\right)$. Similarly, we denote by $\left(\tilde{A}_{\varepsilon}\right)_{h}$ the translation of $\tilde{A}_{\varepsilon}$ where $\tilde{A}_{\varepsilon}$ is extended by zero to the whole of $\mathbb{R}^{N-1} \times(-\infty, 0)$ (the fact that this extension may not be Lipschitz continuous in $\mathbb{R}^{N-1} \times(-\infty, 0)$ is not relevant in what follows).

Fix $v \in H_{0}^{1}\left(W_{j} \times(-\infty, 0)\right)$ such that $\operatorname{supp}(v)$ is a compact set contained in $W_{j} \times(-\infty, 0]$. Then we have for $|h|$ small enough $v_{-h} \in H_{0}^{1}\left(W_{j} \times(-\infty, 0)\right)$ and

$$
\begin{aligned}
& \int_{W_{j} \times(-\infty, 0)}\left(\tilde{A}_{\varepsilon} \nabla\left(\tilde{u}_{h}-\tilde{u}\right)\right) \cdot \nabla v d y \\
& =\int_{W_{j} \times(-\infty, 0)} \tilde{f}_{\varepsilon}\left(v_{-h}-v\right) d y-\int_{W_{j} \times(-\infty, 0)}\left[\left(\left(\tilde{A}_{\varepsilon}\right)_{h}-\tilde{A}_{\varepsilon}\right) \nabla \tilde{u}_{h}\right] \cdot \nabla v d y
\end{aligned}
$$

Now we choose $v=\tilde{u}_{h}-\tilde{u}$ in (51) and we proceed by estimating the term on the right hand side of it. 
In the rest of proof we denote by $C$ positive constants independent of $h$ and $\varepsilon$ which may vary from line to line.

Let $\omega \subset \mathbb{R}^{N-1}$ and $\delta>0$ be such that $\omega$ is open,

$$
\operatorname{supp}(\tilde{u})+h e_{i} \subset \omega \times(-\infty, 0] \text { and }(\bar{\omega} \times(-\infty, 0])+h e_{i} \subset W_{j} \times(-\infty, 0]
$$

for any $h \in(-\delta, \delta)$. In this way we can write

$$
\left|\left(\tilde{A}_{\varepsilon}\right)_{h}(y)-\tilde{A}_{\varepsilon}(y)\right| \leq C|h| \max _{n, m, l \in\{1, \ldots, N\}}\left\|\frac{\partial\left(\tilde{A}_{\varepsilon}\right)_{n m}}{\partial y_{l}}\right\|_{L^{\infty}\left(W_{j} \times(-\infty, 0)\right)} \forall y \in \omega \times(-\infty, 0), \forall|h|<\delta
$$

where $|A|:=\sup _{x \in \mathbb{R}^{N} \backslash\{0\}}|A x| /|x|$ for any $N \times N$ matrix. With this choice of $\omega$ and $\delta$, exploiting (52), (21), the definitions of $\tilde{A}_{\varepsilon}, \Psi_{\varepsilon, j}, \Phi_{\varepsilon, j}$, the fact that $\tilde{A}_{\varepsilon}\left(y^{\prime}, y_{N}\right)=I_{N}$ if $y_{N}<-(\hat{k}+1) \kappa_{\varepsilon}<\tilde{g}_{\varepsilon, j}\left(y^{\prime}\right)-g_{j}\left(y^{\prime}\right)$ (here $I_{N}$ denotes the $N \times N$ identity matrix), and (35) we have

$$
\begin{aligned}
& \left|\int_{W_{j} \times(-\infty, 0)}\left[\left(\left(\tilde{A}_{\varepsilon}\right)_{h}-\tilde{A}_{\varepsilon}\right) \nabla \tilde{u}_{h}\right] \cdot \nabla\left(\tilde{u}_{h}-\tilde{u}\right) d y\right|=\left|\int_{\omega \times(-\infty, 0)}\left[\left(\left(\tilde{A}_{\varepsilon}\right)_{h}-\tilde{A}_{\varepsilon}\right) \nabla \tilde{u}_{h}\right] \cdot \nabla\left(\tilde{u}_{h}-\tilde{u}\right) d y\right| \\
& =\left|\int_{\omega}\left(\int_{-(\hat{k}+1) \kappa_{\varepsilon}}^{0}\left[\left(\left(\tilde{A}_{\varepsilon}\right)_{h}-\tilde{A}_{\varepsilon}\right) \nabla \tilde{u}_{h}\right] \cdot \nabla\left(\tilde{u}_{h}-\tilde{u}\right) d y_{N}\right) d y^{\prime}\right| \\
& \leq C|h| o\left(\kappa_{\varepsilon}^{-1 / 2}\right)\left[\int_{\omega}\left(\int_{-(\hat{k}+1) \kappa_{\varepsilon}}^{0}\left|\nabla\left(\tilde{u}_{h}-\tilde{u}\right)\right|^{2} d y_{N}\right) d y^{\prime}\right]^{1 / 2} \times \\
& \times\left[\int_{\omega}(\hat{k}+1) \kappa_{\varepsilon}\left\|\left|\nabla \tilde{u}_{h}\left(y^{\prime}, \cdot\right)\right|\right\|_{L^{\infty}(-\infty, 0)}^{2} d y^{\prime}\right]^{1 / 2} \\
& \leq C|h| o(1)\left\|\tilde{u}_{h}-\tilde{u}\right\|_{H^{1}\left(W_{j} \times(-\infty, 0)\right)}\left[\int_{\omega}\left\|\left|\nabla \tilde{u}_{h}\left(y^{\prime}, \cdot\right)\right|\right\|_{H^{1}(-\infty, 0)}^{2} d y^{\prime}\right]^{1 / 2} \\
& \leq C|h| o(1) \cdot\left\|\tilde{u}_{h}-\tilde{u}\right\|_{H^{1}\left(W_{j} \times(-\infty, 0)\right)}\|\tilde{u}\|_{H^{2}\left(W_{j} \times(-\infty, 0)\right)}
\end{aligned}
$$

where here and in the rest of the proof, $o\left(\kappa_{\varepsilon}^{-1 / 2}\right)$ and $o(1)$ denote functions depending only on $\varepsilon$ but independent of $h$ which have the prescribed asymptotic behavior as $\varepsilon \rightarrow 0$. We point out that the estimate $\left\|\left|\nabla \tilde{u}_{h}\left(y^{\prime}, \cdot\right)\right|\right\|_{L^{\infty}(-\infty, 0)}^{2} \leq C\left\|\left|\nabla \tilde{u}_{h}\left(y^{\prime}, \cdot\right)\right|\right\|_{H^{1}(-\infty, 0)}^{2}$ has been obtained by exploiting the fact that there exists $M>0$ such that $\operatorname{supp}\left(\tilde{u}_{h}\right) \subset W_{j} \times(-M, 0]$ for any $h \in(-\delta, \delta)$ and applying (35) on the interval $(-M, 0)$. On the other hand we also have that

$$
\left|\int_{W_{j} \times(-\infty, 0)} \tilde{f}_{\varepsilon}\left[\left(\tilde{u}_{h}-\tilde{u}\right)_{-h}-\left(\tilde{u}_{h}-\tilde{u}\right)\right] d y\right| \leq C|h|\left\|\tilde{f}_{\varepsilon}\right\|_{L^{2}\left(W_{j} \times(-\infty, 0)\right)}\left\|\tilde{u}_{h}-\tilde{u}\right\|_{H^{1}\left(W_{j} \times(-\infty, 0)\right)}
$$

Let us consider the left hand side of (51). Since by (21), $\tilde{A}_{\varepsilon} \rightarrow I_{N}$ in $L^{\infty}\left(W_{j} \times(-\infty, 0)\right)$, then up to shrink $\varepsilon_{0}$ if necessary we have

$$
\sum_{n, m=1}^{N}\left(\tilde{A}_{\varepsilon}(y)\right)_{n m} \xi_{n} \xi_{m} \geq \frac{1}{2}|\xi|^{2} \quad \text { for any } y \in W_{j} \times(-\infty, 0), \xi \in \mathbb{R}^{N} \text { and } \varepsilon \in\left(0, \varepsilon_{0}\right] .
$$

Therefore we have that

$$
\begin{aligned}
& \int_{W_{j} \times(-\infty, 0)}\left(\tilde{A}_{\varepsilon}(y) \nabla\left(\tilde{u}_{h}-\tilde{u}\right)\right) \cdot \nabla\left(\tilde{u}_{h}-\tilde{u}\right) d y \\
& \quad \geq \frac{1}{2} \int_{W_{j} \times(-\infty, 0)}\left|\nabla\left(\tilde{u}_{h}-\tilde{u}\right)\right|^{2} d y \geq C\left\|\tilde{u}_{h}-\tilde{u}\right\|_{H^{1}\left(W_{j} \times(-\infty, 0)\right)}^{2} .
\end{aligned}
$$


Combining (53)-(56) with (51) we obtain

$$
\left\|\frac{\tilde{u}_{h}-\tilde{u}}{h}\right\|_{H^{1}\left(W_{j} \times(-\infty, 0)\right)} \leq o(1) \cdot\|\tilde{u}\|_{H^{2}\left(W_{j} \times(-\infty, 0)\right)}+C\left\|\tilde{f}_{\varepsilon}\right\|_{L^{2}\left(W_{j} \times(-\infty, 0)\right)} \quad \forall 0<|h|<\delta, \forall \varepsilon \in\left(0, \varepsilon_{0}\right] .
$$

Passing to the limit as $h \rightarrow 0$ we obtain

$$
\left\|\frac{\partial \tilde{u}}{\partial x_{i}}\right\|_{H^{1}\left(W_{j} \times(-\infty, 0)\right)} \leq o(1) \cdot\|\tilde{u}\|_{H^{2}\left(W_{j} \times(-\infty, 0)\right)}+C\left\|\tilde{f}_{\varepsilon}\right\|_{L^{2}\left(W_{j} \times(-\infty, 0)\right)} \quad \forall \varepsilon \in\left(0, \varepsilon_{0}\right]
$$

for any $i \in\{1, \ldots, N-1\}$.

Since $\tilde{u}$ solves equation (50), using a standard argument (see e.g. page 319 in [20]) one can show that the validity of (57) for any $i \in\{1, \ldots, N-1\}$ implies the validity of (57) also for $i=N$. Therefore we may conclude that up to shrink $\varepsilon_{0}$ if necessary, for any $\varepsilon \in\left(0, \varepsilon_{0}\right]$ we have

$$
\left\|D^{\alpha} \tilde{u}\right\|_{L^{2}\left(W_{j} \times(-\infty, 0)\right)} \leq o(1) \cdot\|\tilde{u}\|_{H^{2}\left(W_{j} \times(-\infty, 0)\right)}+C\left\|\tilde{f}_{\varepsilon}\right\|_{L^{2}\left(W_{j} \times(-\infty, 0)\right)} \forall \alpha \in \mathbb{N}^{N},|\alpha|=2, \forall \varepsilon \in\left(0, \varepsilon_{0}\right]
$$

and, in turn,

$$
\|\tilde{u}\|_{H^{2}\left(W_{j} \times(-\infty, 0)\right)} \leq C\left(\|\tilde{u}\|_{H^{1}\left(W_{j} \times(-\infty, 0)\right)}+\left\|\tilde{f}_{\varepsilon}\right\|_{L^{2}\left(W_{j} \times(-\infty, 0)\right)}\right) .
$$

Testing (50) with $\tilde{u}$, using (55) and the Poincaré inequality in $W_{j} \times(-\infty, 0)$, we deduce that

$$
\|\tilde{u}\|_{H^{1}\left(W_{j} \times(-\infty, 0)\right)} \leq C\left\|\tilde{f}_{\varepsilon}\right\|_{L^{2}\left(W_{j} \times(-\infty, 0)\right)} \quad \forall \varepsilon \in\left(0, \varepsilon_{0}\right]
$$

which combined with (58) gives

$$
\|\tilde{u}\|_{H^{2}\left(W_{j} \times(-\infty, 0)\right)} \leq C\left\|\tilde{f}_{\varepsilon}\right\|_{L^{2}\left(W_{j} \times(-\infty, 0)\right)} \quad \forall \varepsilon \in\left(0, \varepsilon_{0}\right] .
$$

Coming back to $V_{j}$ the last estimate proves (49).

Applying (49) to $\widehat{w}_{\varepsilon, j}$ and using (48) it follows that

$$
\left\|\widehat{w}_{\varepsilon, j}\right\|_{H^{2}(\Omega)} \leq C\left\|L_{\varepsilon} \widehat{w}_{\varepsilon, j}\right\|_{L^{2}\left(\Omega \cap V_{j}\right)} \leq C\|\Delta w\|_{L^{2}\left(\Omega_{\varepsilon}\right)}
$$

for any $\varepsilon \in\left(0, \varepsilon_{0}\right]$.

The proof of the lemma then follows from (43).

As a consequence of Lemma 3.3 we prove the following

Lemma 3.4. Let $\mathcal{A}$ be an atlas. Let $\left\{\Omega_{\varepsilon}\right\}_{0<\varepsilon \leq \varepsilon_{0}}$ be a family of domains of class $C^{1,1}(\mathcal{A})$ and $\Omega$ a domain of class $C^{1,1}(\mathcal{A})$. Assume the validity of condition (21). Then, up to shrink $\varepsilon_{0}$ if necessary,

$$
\|w\|_{H^{2}\left(\Omega_{\varepsilon}\right)} \leq C\|\Delta w\|_{L^{2}\left(\Omega_{\varepsilon}\right)} \quad \text { for any } w \in V\left(\Omega_{\varepsilon}\right) \text { and } \varepsilon \in\left(0, \varepsilon_{0}\right]
$$

where $C>0$ is a constant independent of $w$ and $\varepsilon$.

Proof. Let $w \in V\left(\Omega_{\varepsilon}\right)$. In this proof we use the same notations introduced in the proof of Lemma 3.3. By $C$ we denote positive constants independent of $w$ and $\varepsilon$ which may vary from line to line.

For any $j \in\left\{1, \ldots, s^{\prime}\right\}$ we have

$$
\begin{aligned}
\frac{\partial^{2} w_{j}}{\partial x_{n} \partial x_{m}}(x) & =\sum_{k=1}^{N}\left\{\left[\sum_{l=1}^{N} \frac{\partial^{2} \widehat{w}_{\varepsilon, j}}{\partial x_{l} \partial x_{k}}\left(\Psi_{\varepsilon, j}(x)\right) \frac{\partial\left[\left(\Psi_{\varepsilon, j}(x)\right)_{l}\right]}{\partial x_{m}}\right] \frac{\partial\left[\left(\Psi_{\varepsilon, j}(x)\right)_{k}\right]}{\partial x_{n}}\right. \\
& \left.+\frac{\partial \widehat{w}_{\varepsilon, j}}{\partial x_{k}}\left(\Psi_{\varepsilon, j}(x)\right) \frac{\partial^{2}\left[\left(\Psi_{\varepsilon, j}(x)\right)_{k}\right]}{\partial x_{n} \partial x_{m}}\right\} .
\end{aligned}
$$

By (21), (60), with an argument similar to the one exploited in the proof of Lemma 3.1, we infer

$$
\left\|w_{j}\right\|_{H^{2}\left(\Omega_{\varepsilon}\right)} \leq C\left\|\widehat{w}_{\varepsilon, j}\right\|_{H^{2}(\Omega)} .
$$

The proof of the lemma now follows combining this estimate with (59). 
Thanks to Lemma 3.4 we deduce that, under the assumption 21 , the two norms

$$
u \mapsto\left(\int_{\Omega_{\varepsilon}}|\Delta u|^{2} d x\right)^{1 / 2}, \quad u \mapsto\left(\int_{\Omega_{\varepsilon}}\left|D^{2} u\right|^{2} d x\right)^{1 / 2}
$$

defined on the space $V\left(\Omega_{\varepsilon}\right)$, are uniformly equivalent in the sense that there exists a constant $C>0$ independent of $u$ and $\varepsilon$ such that

$$
\left(\int_{\Omega_{\varepsilon}}|\Delta u|^{2} d x\right)^{1 / 2} \leq C\left(\int_{\Omega_{\varepsilon}}\left|D^{2} u\right|^{2} d x\right)^{1 / 2} \quad \text { and } \quad\left(\int_{\Omega_{\varepsilon}}\left|D^{2} u\right|^{2} d x\right)^{1 / 2} \leq C\left(\int_{\Omega_{\varepsilon}}|\Delta u|^{2} d x\right)^{1 / 2}
$$

for any $u \in V\left(\Omega_{\varepsilon}\right)$.

For this reason, for any $u \in V\left(\Omega_{\varepsilon}\right)$, we put

$$
\|u\|_{V\left(\Omega_{\varepsilon}\right)}:= \begin{cases}\left(\int_{\Omega_{\varepsilon}}|\Delta u|^{2} d x\right)^{1 / 2}, & \text { for problem (2) }, \\ \left(\int_{\Omega_{\varepsilon}}\left|D^{2} u\right|^{2} d x\right)^{1 / 2}, & \text { for problem (5) . }\end{cases}
$$

For the same reason, given a set $A$, we denote by $Q_{A}(\cdot, \cdot)$ and $Q_{A}(\cdot)$ the bilinear form and the quadratic form respectively defined by

$$
Q_{A}(u, v):=\int_{A} \Delta u \Delta v d x \quad \text { and } \quad Q_{A}(u)=Q_{A}(u, u)
$$

or by

$$
Q_{A}(u, v):=\int_{A} D^{2} u: D^{2} v d x \quad \text { and } \quad Q_{A}(u)=Q_{A}(u, u)
$$

depending again on the problem we are treating among (2) or (5).

The next lemma deals with a uniform estimate with respect to domain perturbation of the $H^{1 / 2}$-norm on the boundary in terms of the $H^{1}$-norm of the domain. For simplicity, here and in the sequel we denote the trace of a function and the function itself with the same symbol.

Lemma 3.5. Let $\mathcal{A}$ be an atlas. Let $\left\{\Omega_{\varepsilon}\right\}_{0<\varepsilon \leq \varepsilon_{0}}$ be a family of domains of class $C^{1,1}(\mathcal{A})$ and $\Omega$ a domain of class $C^{1,1}(\mathcal{A})$. Assume the validity of condition (21). Then, up to shrink $\varepsilon_{0}$ if necessary,

$$
\|w\|_{H^{1 / 2}\left(\partial \Omega_{\varepsilon}\right)} \leq C\|w\|_{H^{1}\left(\Omega_{\varepsilon}\right)} \quad \text { for any } w \in H^{1}\left(\Omega_{\varepsilon}\right) \text { and } \varepsilon \in\left(0, \varepsilon_{0}\right]
$$

where $C>0$ is a constant independent of $\varepsilon$.

Proof. In this proof $C$ will denote a positive constant independent of $w$ and $\varepsilon$ which may vary from line to line. The norm in $H^{1 / 2}\left(\partial \Omega_{\varepsilon}\right)$ can be defined locally using the partition of unity $\left\{\psi_{j}\right\}_{1 \leq j \leq s}$ introduced in Subsection 3.2. In other words if $v \in H^{1 / 2}\left(\partial \Omega_{\varepsilon}\right)$, for any $j=1, \ldots, s^{\prime}$, one can define $v_{j}=\psi_{j} v$ on $\partial \Omega_{\varepsilon} \cap V_{j}$ and the function

$$
\bar{v}_{\varepsilon, j}\left(y^{\prime}\right):=v_{j}\left(r_{j}^{-1}\left(y^{\prime}, g_{\varepsilon, j}\left(y^{\prime}\right)\right)\right) \quad \text { for any } y^{\prime} \in W_{j} \subset \mathbb{R}^{N-1} .
$$

Let now $w \in H^{1}\left(\Omega_{\varepsilon}\right)$ and still denote by $w$ its trace on $\partial \Omega_{\varepsilon}$ so that $w$ can be also seen as an element of $H^{1 / 2}\left(\partial \Omega_{\varepsilon}\right)$. Let $\bar{w}_{\varepsilon, j}$ be the corresponding function defined on $W_{j}$ according to (64).

Exploiting in an appropriate way the maps $\Phi_{\varepsilon, j}, \Psi_{\varepsilon, j}$ defined in Subsection 3.2 and the maps $\widetilde{\Phi}_{\varepsilon, j}, \widetilde{\Psi}_{\varepsilon, j}$ defined in the proof of Lemma 3.3, since $w \in H^{1}\left(\Omega_{\varepsilon}\right)$, the function $\bar{w}_{\varepsilon, j}$ can be also interpreted as a function defined on the whole $W_{j} \times(-\infty, 0)$ which belongs to $H^{1}\left(W_{j} \times(-\infty, 0)\right)$ and its support is a compact set contained in $W_{j} \times(-\infty, 0]$.

Now it is clear that the extension-by-zero of $\bar{w}_{\varepsilon, j}$ to the whole $\mathbb{R}^{N-1} \times(-\infty, 0)$, that we still denote by $\bar{w}_{\varepsilon, j}$, belongs to $H^{1}\left(\mathbb{R}^{N-1} \times(-\infty, 0)\right)$ and its trace belongs to $H^{1 / 2}\left(\mathbb{R}^{N-1}\right)$. Since $\bar{w}_{\varepsilon, j}$ is a compactly supported $H^{1 / 2}\left(\mathbb{R}^{N-1}\right)$-function, its norm may be defined in the usual way by mean of the Fourier transform. 
Hence, as possible choice (see [31, Page 83]) for $H^{1 / 2}\left(\partial \Omega_{\varepsilon}\right)$-norm of $w$, we put

$$
\|w\|_{H^{1 / 2}\left(\partial \Omega_{\varepsilon}\right)}=\left(\sum_{j=1}^{s^{\prime}}\left\|\bar{w}_{\varepsilon, j}\right\|_{H^{1 / 2}\left(\mathbb{R}^{N-1}\right)}^{2}\right)^{1 / 2} .
$$

Applying a well-known trace theorem for functions in $H^{1}\left(\mathbb{R}^{N-1} \times(-\infty, 0)\right)$ (see Theorem 5.1, Chap. 2 in [31]) we obtain

$$
\left\|\bar{w}_{\varepsilon, j}\right\|_{H^{1 / 2}\left(\mathbb{R}^{N-1}\right)} \leq C\left\|\bar{w}_{\varepsilon, j}\right\|_{H^{1}\left(\mathbb{R}^{N-1} \times(-\infty, 0)\right)} .
$$

By (21) and direct computation, one can verify that

$$
\left\|\bar{w}_{\varepsilon, j}\right\|_{H^{1}\left(\mathbb{R}^{N-1} \times(-\infty, 0)\right)} \leq C\left\|w_{j}\right\|_{H^{1}\left(\Omega_{\varepsilon}\right)}
$$

The proof of the lemma now follows from (65) and the definition of $w_{j}$.

Lemma 3.6. Let $\mathcal{A}$ be an atlas. Let $\left\{\Omega_{\varepsilon}\right\}_{0<\varepsilon \leq \varepsilon_{0}}$ be a family of domains of class $C^{1,1}(\mathcal{A})$ and $\Omega$ a domain of class $C^{1,1}(\mathcal{A})$. Assume the validity of condition (21). Then

$$
\liminf _{\varepsilon \rightarrow 0} d_{1}^{\varepsilon}>0
$$

where $d_{1}^{\varepsilon}$ is given by (4) with $\Omega_{\varepsilon}$ in place of $\Omega$.

Proof. In this proof we denote by $C$ a general constant independent of $\varepsilon$ which may vary from estimate to estimate. For any $0<\varepsilon \leq \varepsilon_{0}$, let $w_{\varepsilon}$ an eigenfunction of (2) corresponding to $d_{1}^{\varepsilon}$ satisfying $\int_{\partial \Omega_{\varepsilon}}\left(w_{\varepsilon}\right)_{\nu}^{2} d S=$ 1 and let $u_{\varepsilon}:=B_{\varepsilon} w_{\varepsilon}$ with $B_{\varepsilon}$ as in Subsection 3.3. Suppose by contradiction that $\lim _{\text {inf }} \rightarrow 0 d_{1}^{\varepsilon}=0$ so that along a sequence of values of $\varepsilon$ converging to zero we may assume that $d_{1}^{\varepsilon} \rightarrow 0$. For simplicity we write this as $d_{1}^{\varepsilon} \rightarrow 0$ as $\varepsilon \rightarrow 0$. Then we have that along the same sequence $\left\|w_{\varepsilon}\right\|_{V\left(\Omega_{\varepsilon}\right)} \rightarrow 0$ as $\varepsilon \rightarrow 0$ since $\int_{\Omega_{\varepsilon}}\left|\Delta w_{\varepsilon}\right|^{2} d x=d_{1}^{\varepsilon} \int_{\partial \Omega_{\varepsilon}}\left(w_{\varepsilon}\right)_{\nu}^{2} d S$. Applying Lemma 3.3 we deduce that $u_{\varepsilon} \rightarrow 0$ in $V(\Omega)$.

According to (43), for any $1 \leq j \leq s^{\prime}$, we can define $\widehat{w}_{\varepsilon, j}$. Moreover for any $1 \leq j \leq s$ we put $w_{\varepsilon, j}:=\psi_{j} w_{\varepsilon}$. In this way we have that $u_{\varepsilon}=\sum_{j=1}^{s^{\prime}} \widehat{w}_{\varepsilon, j}+\sum_{j=s^{\prime}+1}^{s} w_{\varepsilon, j}$. By direct computation one sees that

$$
\frac{\partial \widehat{w}_{\varepsilon, j}}{\partial x_{k}}(x)=\sum_{n=1}^{N} \frac{\partial w_{\varepsilon, j}}{\partial x_{n}}\left(\Psi_{\varepsilon, j}^{-1}(x)\right) \frac{\partial\left[\left(\Psi_{\varepsilon, j}^{-1}(x)\right)_{n}\right]}{\partial x_{k}} \quad \text { for any } x \in \Omega \cap V_{j} .
$$

We observe that from the definition of $\Psi_{\varepsilon, j}$ and (21) we have

$$
\frac{\partial\left[\left(\Psi_{\varepsilon, j}^{-1}(x)\right)_{n}\right]}{\partial x_{k}} \rightarrow \delta_{n k} \quad \text { uniformly in } V_{j} \cap \Omega \text { as } \varepsilon \rightarrow 0
$$

for any $n, k \in\{1, \ldots, N\}$.

On the other hand, since $\int_{\partial \Omega_{\varepsilon}}\left|\nabla w_{\varepsilon}\right|^{2} d S=\int_{\partial \Omega_{\varepsilon}}\left(w_{\varepsilon}\right)_{\nu}^{2} d S=1$ being $w_{\varepsilon} \equiv 0$ on $\partial \Omega_{\varepsilon}$, for any $j \in$ $\left\{1, \ldots, s^{\prime}\right\}$ and $k \in\{1, \ldots, N\}, \int_{\partial \Omega_{\varepsilon}}\left(\frac{\partial w_{\varepsilon, j}}{\partial x_{k}}\right)^{2} d S$ remains bounded as $\varepsilon \rightarrow 0$. Moreover, using the local parametrizations of $\partial \Omega_{\varepsilon}$ and $\partial \Omega$ combined with $(21)$, we also have

$$
\int_{\partial \Omega \cap V_{j}}\left(\frac{\partial w_{\varepsilon, j}}{\partial x_{k}}\left(\Psi_{\varepsilon, j}^{-1}(x)\right)\right)^{2} d S=\int_{\partial \Omega_{\varepsilon} \cap V_{j}}\left(\frac{\partial w_{\varepsilon, j}}{\partial x_{k}}\right)^{2} d S+o(1)=O(1) \quad \text { as } \varepsilon \rightarrow 0 .
$$

Combining (68) with (67), for any $j \in\left\{1, \ldots, s^{\prime}\right\}$ and $n, k \in\{1, \ldots, N\}$, we obtain

$$
\int_{\partial \Omega \cap V_{j}}\left(\frac{\partial w_{\varepsilon, j}}{\partial x_{n}}\left(\Psi_{\varepsilon, j}^{-1}(x)\right)\right)^{2}\left(\frac{\partial\left[\left(\Psi_{\varepsilon, j}^{-1}(x)\right)_{n}\right]}{\partial x_{k}}\right)^{2} d S \rightarrow 0 \quad \text { as } \varepsilon \rightarrow 0
$$

if $n \neq k$ and

$$
\int_{\partial \Omega \cap V_{j}}\left(\frac{\partial w_{\varepsilon, j}}{\partial x_{k}}\left(\Psi_{\varepsilon, j}^{-1}(x)\right)\right)^{2}\left(\frac{\partial\left[\left(\Psi_{\varepsilon, i}^{-1}(x)\right)_{k}\right]}{\partial x_{k}}\right)^{2} d S=\int_{\partial \Omega \cap V_{j}}\left(\frac{\partial w_{\varepsilon, j}}{\partial x_{k}}\left(\Psi_{\varepsilon, j}^{-1}(x)\right)\right)^{2} d S+o(1) \quad \text { as } \varepsilon \rightarrow 0 .
$$


From the proof of Lemma 3.3 one can deduce that not only $u_{\varepsilon} \rightarrow 0$ in $V(\Omega)$ but also $\widehat{w}_{\varepsilon, j} \rightarrow 0$ in $V(\Omega)$, for any $j \in\left\{1, \ldots, s^{\prime}\right\}$. Hence from the continuity of the trace map 177 we also have that $\frac{\partial \widehat{w}_{\varepsilon, j}}{\partial x_{k}} \rightarrow 0$ in $L^{2}(\partial \Omega)$ as $\varepsilon \rightarrow 0$. From this and (66), [69], it follows that $\frac{\partial w_{\varepsilon, j}}{\partial x_{k}}\left(\Psi_{\varepsilon, j}^{-1}(x)\right) \frac{\partial\left[\left(\Psi_{\varepsilon, j}^{-1}(x)\right)_{k}\right]}{\partial x_{k}} \rightarrow 0$ in $L^{2}(\partial \Omega)$ as $\varepsilon \rightarrow 0$. This, together with (68) and (70) yields

$$
\int_{\partial \Omega_{\varepsilon}}\left(\frac{\partial w_{\varepsilon, j}}{\partial x_{k}}\right)^{2} d S \rightarrow 0 \quad \text { as } \varepsilon \rightarrow 0 \quad \text { for any } j \in\left\{1, \ldots, s^{\prime}\right\} \text { and } k \in\{1, \ldots, N\}
$$

and, in turn, $\int_{\partial \Omega_{\varepsilon}}\left|\nabla w_{\varepsilon}\right|^{2} d S=o(1)$ as $\varepsilon \rightarrow 0$, a contradiction.

Now, we introduce some notations which will be fundamental in the proofs of the next lemmas. For any $y \in \Psi_{\varepsilon, j}\left(\partial \Omega_{\varepsilon} \cap V_{i} \cap V_{j}\right)$ we put $\Theta_{\varepsilon, i, j}(y):=\Psi_{\varepsilon, i}\left(\Psi_{\varepsilon, j}^{-1}(y)\right)$ in order to define

$$
\Theta_{\varepsilon, i, j}: \Psi_{\varepsilon, j}\left(\partial \Omega_{\varepsilon} \cap V_{i} \cap V_{j}\right) \rightarrow \Theta_{\varepsilon, i, j}\left(\Psi_{\varepsilon, j}\left(\partial \Omega_{\varepsilon} \cap V_{i} \cap V_{j}\right)\right)
$$

as a diffeomorphism between two open subsets of the manifold $\partial \Omega$.

For any $k \in\left\{1, \ldots, s^{\prime}\right\}$ we define the local charts $\Gamma_{k}: \partial \Omega \cap V_{k} \rightarrow W_{k} \subset \mathbb{R}^{N-1}$ where $\Gamma_{k}(y):=P\left(r_{k}(y)\right)$ and $P: \mathbb{R}^{N} \rightarrow \mathbb{R}^{N-1}$ is the projection $\left(x^{\prime}, x_{N}\right) \mapsto x^{\prime}$. We observe that $\Gamma_{k}^{-1}: W_{k} \rightarrow \partial \Omega \cap V_{k}$ satisfies $\Gamma_{k}^{-1}\left(z^{\prime}\right)=r_{k}^{-1}\left(z^{\prime}, g_{k}\left(z^{\prime}\right)\right)$ for any $z^{\prime} \in W_{k}$. Next we introduce the map

$$
\Upsilon_{\varepsilon, i, j}: \Gamma_{j}\left(\Psi_{\varepsilon, j}\left(\partial \Omega_{\varepsilon} \cap V_{i} \cap V_{j}\right)\right) \rightarrow \Upsilon_{\varepsilon, i, j}\left(\Gamma_{j}\left(\Psi_{\varepsilon, j}\left(\partial \Omega_{\varepsilon} \cap V_{i} \cap V_{j}\right)\right)\right)
$$

where $\Upsilon_{\varepsilon, i, j}\left(z^{\prime}\right):=\Gamma_{i}\left(\Theta_{\varepsilon, i, j}\left(\Gamma_{j}^{-1}\left(z^{\prime}\right)\right)\right)$ for any $z^{\prime} \in \Gamma_{j}\left(\Psi_{\varepsilon, j}\left(\partial \Omega_{\varepsilon} \cap V_{i} \cap V_{j}\right)\right)$.

Lemma 3.7. Let $\mathcal{A}$ be an atlas. Let $\left\{\Omega_{\varepsilon}\right\}_{0<\varepsilon \leq \varepsilon_{0}}$ be a family of domains of class $C^{1,1}(\mathcal{A})$ and $\Omega$ a domain of class $C^{1,1}(\mathcal{A})$. Assume the validity of condition (21).

(i) Let $i, j \in\left\{1, \ldots, s^{\prime}\right\}$. Let $\left\{f_{\varepsilon}\right\}_{0<\varepsilon<\varepsilon_{0}} \subset L^{2}\left(\mathbb{R}^{N-1}\right)$ be such that $\operatorname{supp}\left(f_{\varepsilon}\right) \subset \Gamma_{i}\left(\partial \Omega \cap V_{i}\right)$ for any $\varepsilon \in\left(0, \varepsilon_{0}\right]$ and let $f \in L^{2}\left(\mathbb{R}^{N-1}\right)$ be such that $f_{\varepsilon} \rightarrow f$ in $L^{2}\left(\mathbb{R}^{N-1}\right)$ as $\varepsilon \rightarrow 0$. Let $\Upsilon_{\varepsilon, i, j}$ be as in (72). Define

$$
\tilde{f}_{\varepsilon}\left(z^{\prime}\right):= \begin{cases}f_{\varepsilon}\left(\Upsilon_{\varepsilon, i, j}\left(z^{\prime}\right)\right) & \text { if } z^{\prime} \in \Gamma_{j}\left(\Psi_{\varepsilon, j}\left(\partial \Omega_{\varepsilon} \cap V_{i} \cap V_{j}\right)\right), \\ 0 & \text { if } z^{\prime} \in \mathbb{R}^{N-1} \backslash \Gamma_{j}\left(\Psi_{\varepsilon, j}\left(\partial \Omega_{\varepsilon} \cap V_{i} \cap V_{j}\right)\right),\end{cases}
$$

for any $\varepsilon \in\left(0, \varepsilon_{0}\right]$ and

$$
\tilde{f}\left(z^{\prime}\right):= \begin{cases}f\left(\Gamma_{i}\left(\Gamma_{j}^{-1}\left(z^{\prime}\right)\right)\right) & \text { if } z^{\prime} \in \Gamma_{j}\left(\partial \Omega \cap V_{i} \cap V_{j}\right), \\ 0 & \text { if } z^{\prime} \in \mathbb{R}^{N-1} \backslash \Gamma_{j}\left(\partial \Omega \cap V_{i} \cap V_{j}\right) .\end{cases}
$$

Then $\tilde{f}_{\varepsilon} \rightarrow \tilde{f}$ in $L^{2}\left(\mathbb{R}^{N-1}\right)$ as $\varepsilon \rightarrow 0$.

(ii) Let $\left\{\omega_{\varepsilon}\right\}_{0<\varepsilon \leq \varepsilon_{0}} \subset L^{2}(\partial \Omega)$ be such that $\operatorname{supp}\left(\omega_{\varepsilon}\right) \subset \partial \Omega \cap V_{i}$ for any $\varepsilon \in\left(0, \varepsilon_{0}\right]$, for some $i \in$ $\left\{1, \ldots, s^{\prime}\right\}$. Suppose that there exists $\omega \in L^{2}(\partial \Omega)$ such that $\omega_{\varepsilon} \rightarrow \omega$ in $L^{2}(\partial \Omega)$ as $\varepsilon \rightarrow 0$. For $j \in\left\{1, \ldots, s^{\prime}\right\}$ let $\Theta_{\varepsilon, i, j}$ be as in (71). For any $\varepsilon \in\left(0, \varepsilon_{0}\right]$ define the function

$$
\tilde{\omega}_{\varepsilon}(y):= \begin{cases}\omega_{\varepsilon}\left(\Theta_{\varepsilon, i, j}(y)\right) & \text { if } y \in \Psi_{\varepsilon, j}\left(\partial \Omega_{\varepsilon} \cap V_{i} \cap V_{j}\right), \\ 0 & \text { if } y \in \partial \Omega \backslash \Psi_{\varepsilon, j}\left(\partial \Omega_{\varepsilon} \cap V_{i} \cap V_{j}\right) .\end{cases}
$$

Then $\tilde{\omega}_{\varepsilon} \rightarrow \omega \chi \partial \Omega \cap V_{i} \cap V_{j}$ in $L^{2}(\partial \Omega)$ as $\varepsilon \rightarrow 0$.

Proof. We divide the proof of the lemma into three steps. Throughout this proof, $C$ denotes a constant independent of $\varepsilon$ which may vary from line to line.

Step 1. In this step we prove (i) when $f_{\varepsilon} \equiv f$ for any $\varepsilon \in\left(0, \varepsilon_{0}\right]$.

For any $\sigma>0$ let $\varphi_{\sigma} \in C^{0}\left(\mathbb{R}^{N-1}\right)$ be such that $\left\|f-\varphi_{\sigma}\right\|_{L^{2}\left(\mathbb{R}^{N-1}\right)}<\sigma$ and $\operatorname{supp}\left(\varphi_{\sigma}\right) \subset \Gamma_{i}\left(\partial \Omega \cap V_{i}\right)$.

Similarly to the statement of the lemma we define

$$
\tilde{\varphi}_{\sigma, \varepsilon}\left(z^{\prime}\right):= \begin{cases}\varphi_{\sigma}\left(\Upsilon_{\varepsilon, i, j}\left(z^{\prime}\right)\right) & \text { if } z^{\prime} \in \Gamma_{j}\left(\Psi_{\varepsilon, j}\left(\partial \Omega_{\varepsilon} \cap V_{i} \cap V_{j}\right)\right), \\ 0 & \text { if } z^{\prime} \in \mathbb{R}^{N-1} \backslash \Gamma_{j}\left(\Psi_{\varepsilon, j}\left(\partial \Omega_{\varepsilon} \cap V_{i} \cap V_{j}\right)\right) .\end{cases}
$$


and

$$
\tilde{\varphi}_{\sigma}\left(z^{\prime}\right):= \begin{cases}\varphi_{\sigma}\left(\Gamma_{i}\left(\Gamma_{j}^{-1}\left(z^{\prime}\right)\right)\right) & \text { if } z^{\prime} \in \Gamma_{j}\left(\partial \Omega \cap V_{i} \cap V_{j}\right), \\ 0 & \text { if } z^{\prime} \in \mathbb{R}^{N-1} \backslash \Gamma_{j}\left(\partial \Omega \cap V_{i} \cap V_{j}\right) .\end{cases}
$$

By (21) we deduce that $\tilde{\varphi}_{\varepsilon, \sigma} \rightarrow \tilde{\varphi}_{\sigma}$ almost everywhere in $\mathbb{R}^{N-1}$ and that up to shrink $\varepsilon_{0}$ if necessary, there exists a compact set in $\mathbb{R}^{N-1}$ which contains the support of $\tilde{\varphi}_{\sigma, \varepsilon}$ for any $\varepsilon \in\left(0, \varepsilon_{0}\right]$. Therefore by the Dominated Convergence Theorem we have that

$$
\tilde{\varphi}_{\sigma, \varepsilon} \rightarrow \tilde{\varphi}_{\sigma} \quad \text { in } L^{2}\left(\mathbb{R}^{N-1}\right) \text { as } \varepsilon \rightarrow 0 .
$$

On the other hand with a change of variable, by 21 we obtain

$$
\begin{aligned}
\left\|\tilde{f}_{\varepsilon}-\tilde{\varphi}_{\sigma, \varepsilon}\right\|_{L^{2}\left(\mathbb{R}^{N-1}\right)}^{2} & =\int_{\Gamma_{j}\left(\Psi_{\varepsilon, j}\left(\partial \Omega_{\varepsilon} \cap V_{i} \cap V_{j}\right)\right)}\left|f\left(\Upsilon_{\varepsilon, i, j}\left(z^{\prime}\right)\right)-\varphi_{\sigma}\left(\Upsilon_{\varepsilon, i, j}\left(z^{\prime}\right)\right)\right|^{2} d z^{\prime} \\
& \leq C \int_{\Gamma_{i}\left(\partial \Omega \cap V_{i}\right)}\left|f\left(x^{\prime}\right)-\varphi_{\sigma}\left(x^{\prime}\right)\right|^{2} d x^{\prime} \leq C \sigma^{2}
\end{aligned}
$$

and

$$
\begin{aligned}
\left\|\tilde{f}-\tilde{\varphi}_{\sigma}\right\|_{L^{2}\left(\mathbb{R}^{N-1}\right)}^{2} & =\int_{\Gamma_{j}\left(\partial \Omega \cap V_{i} \cap V_{j}\right)}\left|f\left(\Gamma_{i}\left(\Gamma_{j}^{-1}\left(z^{\prime}\right)\right)\right)-\varphi_{\sigma}\left(\Gamma_{i}\left(\Gamma_{j}^{-1}\left(z^{\prime}\right)\right)\right)\right|^{2} d z^{\prime} \\
& \leq C \int_{\Gamma_{i}\left(\partial \Omega \cap V_{i}\right)}\left|f\left(x^{\prime}\right)-\varphi_{\sigma}\left(x^{\prime}\right)\right|^{2} d x^{\prime} \leq C \sigma^{2} .
\end{aligned}
$$

Combining (74), (75) we have

$$
\begin{aligned}
& \left\|\tilde{f}_{\varepsilon}-\tilde{f}\right\|_{L^{2}\left(\mathbb{R}^{N-1}\right)} \\
& \leq\left\|\tilde{f}_{\varepsilon}-\tilde{\varphi}_{\sigma, \varepsilon}\right\|_{L^{2}\left(\mathbb{R}^{N-1}\right)}+\left\|\tilde{\varphi}_{\sigma, \varepsilon}-\tilde{\varphi}_{\sigma}\right\|_{L^{2}\left(\mathbb{R}^{N-1}\right)}+\left\|\tilde{\varphi}_{\sigma}-\tilde{f}\right\|_{L^{2}\left(\mathbb{R}^{N-1}\right)} \leq 2 \sqrt{C} \sigma+\left\|\tilde{\varphi}_{\sigma, \varepsilon}-\tilde{\varphi}_{\sigma}\right\|_{L^{2}\left(\mathbb{R}^{N-1}\right)}
\end{aligned}
$$

and the proof of Step 1 follows by (73) and the arbitrariness of $\sigma$.

Step 2. In this step we prove (i) in the general case. To this purpose we define

$$
\tilde{g}_{\varepsilon}\left(z^{\prime}\right):= \begin{cases}f\left(\Upsilon_{\varepsilon, i, j}\left(z^{\prime}\right)\right) & \text { if } z^{\prime} \in \Gamma_{j}\left(\Psi_{\varepsilon, j}\left(\partial \Omega_{\varepsilon} \cap V_{i} \cap V_{j}\right)\right), \\ 0 & \text { if } z^{\prime} \in \mathbb{R}^{N-1} \backslash \Gamma_{j}\left(\Psi_{\varepsilon, j}\left(\partial \Omega_{\varepsilon} \cap V_{i} \cap V_{j}\right)\right)\end{cases}
$$

for any $\varepsilon \in\left(0, \varepsilon_{0}\right]$. By Step 1 we have

$$
\tilde{g}_{\varepsilon} \rightarrow \tilde{f} \quad \text { in } L^{2}\left(\mathbb{R}^{N-1}\right) \text { as } \varepsilon \rightarrow 0 .
$$

On the other hand by (21),

$$
\begin{aligned}
\left\|\tilde{f}_{\varepsilon}-\tilde{g}_{\varepsilon}\right\|_{L^{2}\left(\mathbb{R}^{N-1}\right)}^{2} & =\int_{\Gamma_{j}\left(\Psi_{\varepsilon, j}\left(\partial \Omega_{\varepsilon} \cap V_{i} \cap V_{j}\right)\right)}\left|f_{\varepsilon}\left(\Upsilon_{\varepsilon, i, j}\left(z^{\prime}\right)\right)-f\left(\Upsilon_{\varepsilon, i, j}\left(z^{\prime}\right)\right)\right|^{2} d z^{\prime} \\
& \leq C \int_{\Gamma_{i}\left(\partial \Omega \cap V_{i}\right)}\left|f_{\varepsilon}\left(x^{\prime}\right)-f\left(x^{\prime}\right)\right|^{2} d x^{\prime}=C\left\|f_{\varepsilon}-f\right\|_{L^{2}\left(\mathbb{R}^{N-1}\right)} .
\end{aligned}
$$

Combining (76) and (77) we obtain

$$
\left\|\tilde{f}_{\varepsilon}-\tilde{f}\right\|_{L^{2}\left(\mathbb{R}^{N-1}\right)} \leq\left\|\tilde{f}_{\varepsilon}-\tilde{g}_{\varepsilon}\right\|_{L^{2}\left(\mathbb{R}^{N-1}\right)}+\left\|\tilde{g}_{\varepsilon}-\tilde{f}\right\|_{L^{2}\left(\mathbb{R}^{N-1}\right)}
$$

the proof of Step 2 follows.

Step 3. In this step we prove (ii). We set

$$
\begin{aligned}
f_{\varepsilon}\left(z^{\prime}\right) & = \begin{cases}\omega_{\varepsilon}\left(\Gamma_{i}^{-1}\left(z^{\prime}\right)\right) & \text { if } z^{\prime} \in \Gamma_{i}\left(\partial \Omega \cap V_{i}\right), \\
0 & \text { if } z^{\prime} \in \mathbb{R}^{N-1} \backslash \Gamma_{i}\left(\partial \Omega \cap V_{i}\right),\end{cases} \\
f\left(z^{\prime}\right) & = \begin{cases}\omega\left(\Gamma_{i}^{-1}\left(z^{\prime}\right)\right) & \text { if } z^{\prime} \in \Gamma_{i}\left(\partial \Omega \cap V_{i}\right), \\
0 & \text { if } z^{\prime} \in \mathbb{R}^{N-1} \backslash \Gamma_{i}\left(\partial \Omega \cap V_{i}\right),\end{cases}
\end{aligned}
$$


and

$$
\begin{gathered}
\phi_{\varepsilon}(y)= \begin{cases}f_{\varepsilon}\left(\Gamma_{i}\left(\Theta_{\varepsilon, i, j}(y)\right)\right) & \text { if } y \in \Psi_{\varepsilon, j}\left(\partial \Omega_{\varepsilon} \cap V_{i} \cap V_{j}\right), \\
0 & \text { if } y \in \partial \Omega \backslash \Psi_{\varepsilon, j}\left(\partial \Omega_{\varepsilon} \cap V_{i} \cap V_{j}\right),\end{cases} \\
\phi(y)= \begin{cases}f\left(\Gamma_{i}(y)\right) & \text { if } y \in \partial \Omega \cap V_{i} \cap V_{j}, \\
0 & \text { if } y \in \partial \Omega \backslash\left(V_{i} \cap V_{j}\right) .\end{cases}
\end{gathered}
$$

We observe that, using the notation of statement (i), $\tilde{f}_{\varepsilon}\left(z^{\prime}\right)=\phi_{\varepsilon}\left(\Gamma_{j}^{-1}\left(z^{\prime}\right)\right)$ and $\tilde{f}\left(z^{\prime}\right)=\phi\left(\Gamma_{j}^{-1}\left(z^{\prime}\right)\right)$ for any $z^{\prime} \in \Gamma_{j}\left(\partial \Omega \cap V_{j}\right)$. Moreover,

$$
\begin{aligned}
& \int_{\partial \Omega}\left|\phi_{\varepsilon}-\phi\right|^{2} d S=\int_{\partial \Omega \cap V_{j}}\left|\phi_{\varepsilon}-\phi\right|^{2} d S=\int_{\Gamma_{j}\left(\partial \Omega \cap V_{j}\right)}\left|\phi_{\varepsilon}\left(\Gamma_{j}^{-1}\left(z^{\prime}\right)\right)-\phi\left(\Gamma_{j}^{-1}\left(z^{\prime}\right)\right)\right|^{2} \sqrt{1+\left|\nabla_{z^{\prime}} g_{j}\left(z^{\prime}\right)\right|^{2}} d z^{\prime} \\
& \leq C \int_{\mathbb{R}^{N-1}}\left|\tilde{f}_{\varepsilon}\left(z^{\prime}\right)-\tilde{f}\left(z^{\prime}\right)\right|^{2} d z^{\prime}=C\left\|\tilde{f}_{\varepsilon}-\tilde{f}\right\|_{L^{2}\left(\mathbb{R}^{N-1}\right)} .
\end{aligned}
$$

Then by (i) we have that $\phi_{\varepsilon} \rightarrow \phi$ in $L^{2}(\partial \Omega)$ as $\varepsilon \rightarrow 0$. In order to conclude it suffices to observe that $\tilde{\omega}_{\varepsilon}$ coincides with $\phi_{\varepsilon}$ and $\omega \chi_{\partial \Omega \cap V_{i} \cap v_{j}}$ coincides with $\phi$.

Next we prove

Lemma 3.8. Let $\mathcal{A}$ be an atlas. Let $\left\{\Omega_{\varepsilon}\right\}_{0<\varepsilon \leq \varepsilon_{0}}$ be a family of domains of class $C^{1,1}(\mathcal{A})$ and $\Omega$ a domain of class $C^{1,1}(\mathcal{A})$. Assume the validity of condition [21). Let $w_{\varepsilon} \in V\left(\Omega_{\varepsilon}\right)$ with $0<\varepsilon \leq \varepsilon_{0}$, and $w \in V(\Omega)$ be such that $w_{\varepsilon} \stackrel{E}{\longrightarrow} w$. If we put $u_{\varepsilon}:=S_{\Delta, \varepsilon} w_{\varepsilon}$ and $u:=S_{\Delta} w$ or $u_{\varepsilon}:=S_{D^{2}, \varepsilon} w_{\varepsilon}$ and $u:=S_{D^{2}} w$, then $B_{\varepsilon} u_{\varepsilon} \rightarrow u$ in $V(\Omega)$ as $\varepsilon \rightarrow 0$.

Proof. We prove the lemma by using an argument which works for both problems (2) and (5). In doing this, we use the notation introduced in (61), 62), (63). We divide the proof of the lemma in several steps.

Step 1. In this step we prove that the $V\left(\Omega_{\varepsilon}\right)$-norm of $u_{\varepsilon}$ is uniformly bounded with respect to $\varepsilon \in\left(0, \varepsilon_{0}\right]$.

First of all we observe that

$$
\begin{aligned}
\left\|u_{\varepsilon}\right\|_{V\left(\Omega_{\varepsilon}\right)}^{2} & =\int_{\partial \Omega_{\varepsilon}}\left(w_{\varepsilon}\right)_{\nu}\left(u_{\varepsilon}\right)_{\nu} d S \leq\left(\int_{\partial \Omega_{\varepsilon}}\left(w_{\varepsilon}\right)_{\nu}^{2} d S\right)^{1 / 2}\left(\int_{\partial \Omega_{\varepsilon}}\left(u_{\varepsilon}\right)_{\nu}^{2} d S\right)^{1 / 2} \\
& \leq\left(d_{1}^{\varepsilon}\right)^{-1}\left(\int_{\Omega_{\varepsilon}}\left|\Delta w_{\varepsilon}\right|^{2} d x\right)^{1 / 2}\left(\int_{\Omega_{\varepsilon}}\left|\Delta u_{\varepsilon}\right|^{2} d x\right)^{1 / 2} \leq N\left(d_{1}^{\varepsilon}\right)^{-1}\left\|w_{\varepsilon}\right\|_{V\left(\Omega_{\varepsilon}\right)}\left\|u_{\varepsilon}\right\|_{V\left(\Omega_{\varepsilon}\right)} .
\end{aligned}
$$

from which it follows that $\left\|u_{\varepsilon}\right\|_{V\left(\Omega_{\varepsilon}\right)} \leq N\left(d_{1}^{\varepsilon}\right)^{-1}\left\|w_{\varepsilon}\right\|_{V\left(\Omega_{\varepsilon}\right)}$.

Therefore, by Lemma 3.6 and the fact that $\varepsilon \mapsto\left\|w_{\varepsilon}\right\|_{V\left(\Omega_{\varepsilon}\right)}$ is bounded in view of Lemma3.1(ii), it follows that there exists a constant $C>0$ independent of $\varepsilon$ such that

$$
\left\|u_{\varepsilon}\right\|_{V\left(\Omega_{\varepsilon}\right)} \leq C \quad \text { for any } \varepsilon \in\left(0, \varepsilon_{0}\right] .
$$

Step 2. We note that $\left\{B_{\varepsilon} u_{\varepsilon}\right\}_{0<\varepsilon \leq \varepsilon_{0}}$ is bounded in $V(\Omega)$ and, in particular, that it is weakly convergent in $V(\Omega)$ along a sequence $\varepsilon_{n} \downarrow 0$ as $n \rightarrow+\infty$. Indeed, applying Lemma 3.3 to $\left\{u_{\varepsilon}\right\}_{0<\varepsilon \leq \varepsilon_{0}}$, it follows that $\left\{B_{\varepsilon} u_{\varepsilon}\right\}_{0<\varepsilon \leq \varepsilon_{0}}$ is bounded in $V(\Omega)$ up to shrink $\varepsilon_{0}$ if necessary. Thus, for a sequence $\varepsilon_{n} \downarrow 0$ we have that there exists $\tilde{u} \in V(\Omega)$ such that $B_{\varepsilon_{n}} u_{\varepsilon_{n}} \rightarrow \tilde{u}$ in $V(\Omega)$. For simplicity in the sequel we only write $B_{\varepsilon} u_{\varepsilon} \rightarrow \tilde{u}$ as $\varepsilon \rightarrow 0$ for denoting this convergence along the sequence $\left\{\varepsilon_{n}\right\}$. The purpose of the next two steps is to prove that $\tilde{u}=u$. This will be done passing to the limit in the following identity

$$
Q_{\Omega_{\varepsilon}}\left(u_{\varepsilon}, E_{\varepsilon} \varphi\right)=\int_{\partial \Omega_{\varepsilon}}\left(w_{\varepsilon}\right)_{\nu}\left(E_{\varepsilon} \varphi\right)_{\nu} d S \quad \text { for any } \varphi \in V(\Omega) .
$$

Step 3. In this step we pass to the limit in the left hand side of (80).

We define $K_{\varepsilon}:=\left(\bigcup_{j=1}^{s^{\prime}} r_{j}^{-1}\left(K_{\varepsilon, j}\right)\right) \cup\left(\bigcup_{j=s^{\prime}+1}^{s} V_{j}\right)$ and we split the left hand side of $[80$ ) into two parts

$$
Q_{\Omega_{\varepsilon}}\left(u_{\varepsilon}, E_{\varepsilon} \varphi\right)=Q_{K_{\varepsilon}}\left(u_{\varepsilon}, E_{\varepsilon} \varphi\right)+Q_{\Omega_{\varepsilon} \backslash K_{\varepsilon}}\left(u_{\varepsilon}, E_{\varepsilon} \varphi\right) .
$$

Here $K_{\varepsilon, j}$ denotes the set defined in Subsection 3.2 . 
We recall that $\varphi_{j}=\psi_{j} \varphi$ and that $E_{\varepsilon} \varphi=\sum_{j=1}^{s^{\prime}} \tilde{\varphi}_{\varepsilon, j}+\sum_{j=s^{\prime}+1}^{s} \varphi_{j}$. Thus

$$
\begin{aligned}
& Q_{K_{\varepsilon}}\left(u_{\varepsilon}, E_{\varepsilon} \varphi\right)=\sum_{j=1}^{s^{\prime}} Q_{K_{\varepsilon}}\left(u_{\varepsilon}, \tilde{\varphi}_{\varepsilon, j}\right)+\sum_{j=s^{\prime}+1}^{s} Q_{K_{\varepsilon}}\left(u_{\varepsilon}, \varphi_{j}\right)=\sum_{j=1}^{s^{\prime}} Q_{K_{\varepsilon} \cap V_{j}}\left(u_{\varepsilon}, \tilde{\varphi}_{\varepsilon, j}\right)+\sum_{j=s^{\prime}+1}^{s} Q_{K_{\varepsilon}}\left(u_{\varepsilon}, \varphi_{j}\right) \\
& =\sum_{j=1}^{s^{\prime}} Q_{\left(K_{\varepsilon} \cap V_{j}\right) \backslash r_{j}^{-1}\left(K_{\varepsilon, j}\right)}\left(u_{\varepsilon}, \tilde{\varphi}_{\varepsilon, j}\right)+\sum_{j=1}^{s^{\prime}} Q_{r_{j}^{-1}\left(K_{\varepsilon, j}\right)}\left(u_{\varepsilon}, \tilde{\varphi}_{\varepsilon, j}\right)+\sum_{j=s^{\prime}+1}^{s} Q_{K_{\varepsilon}}\left(u_{\varepsilon}, \varphi_{j}\right) .
\end{aligned}
$$

But

$$
\left|Q_{\left(K_{\varepsilon} \cap V_{j}\right) \backslash r_{j}^{-1}\left(K_{\varepsilon, j}\right)}\left(u_{\varepsilon}, \tilde{\varphi}_{\varepsilon, j}\right)\right| \leq\left(Q_{\left(K_{\varepsilon} \cap V_{j}\right) \backslash r_{j}^{-1}\left(K_{\varepsilon, j}\right)}\left(u_{\varepsilon}\right)\right)^{1 / 2}\left(Q_{\left(K_{\varepsilon} \cap V_{j}\right) \backslash r_{j}^{-1}\left(K_{\varepsilon, j}\right)}\left(\tilde{\varphi}_{\varepsilon, j}\right)\right)^{1 / 2}=o(1)
$$

since $Q_{\left(K_{\varepsilon} \cap V_{j}\right) \backslash r_{j}^{-1}\left(K_{\varepsilon, j}\right)}\left(u_{\varepsilon}\right)=O(1)$ thanks to $(79)$ and $Q_{\left(K_{\varepsilon} \cap V_{j}\right) \backslash r_{j}^{-1}\left(K_{\varepsilon, j}\right)}\left(\tilde{\varphi}_{\varepsilon, j}\right)=o(1)$ as one can see by proceeding as for (32). Similarly we have $Q_{\left(K_{\varepsilon} \cap V_{j}\right) \backslash r_{j}^{-1}\left(K_{\varepsilon, j}\right)}\left(u_{\varepsilon}, \varphi_{j}\right)=o(1)$. Hence

$$
\begin{aligned}
& Q_{K_{\varepsilon}}\left(u_{\varepsilon}, E_{\varepsilon} \varphi\right)=\sum_{j=1}^{s^{\prime}} Q_{r_{j}^{-1}\left(K_{\varepsilon, j}\right)}\left(u_{\varepsilon}, \tilde{\varphi}_{\varepsilon, j}\right)+\sum_{j=s^{\prime}+1}^{s} Q_{K_{\varepsilon}}\left(u_{\varepsilon}, \varphi_{j}\right)+o(1) \\
& =\sum_{j=1}^{s^{\prime}} Q_{r_{j}^{-1}\left(K_{\varepsilon, j}\right)}\left(u_{\varepsilon}, \varphi_{j}\right)+\sum_{j=s^{\prime}+1}^{s} Q_{K_{\varepsilon}}\left(u_{\varepsilon}, \varphi_{j}\right)+o(1) \\
& =\sum_{j=1}^{s^{\prime}} Q_{K_{\varepsilon}}\left(u_{\varepsilon}, \varphi_{j}\right)+\sum_{j=s^{\prime}+1}^{s} Q_{K_{\varepsilon}}\left(u_{\varepsilon}, \varphi_{j}\right)+o(1)=Q_{K_{\varepsilon}}\left(u_{\varepsilon}, \varphi\right)+o(1) .
\end{aligned}
$$

In a similar way one can treat $Q_{K_{\varepsilon}}\left(B_{\varepsilon} u_{\varepsilon}, \varphi\right)$ :

(83)

$$
\begin{aligned}
& Q_{K_{\varepsilon}}\left(B_{\varepsilon} u_{\varepsilon}, \varphi\right)=\sum_{j=1}^{s^{\prime}} Q_{K_{\varepsilon}}\left(\widehat{u}_{\varepsilon, j}, \varphi\right)+\sum_{j=s^{\prime}+1}^{s} Q_{K_{\varepsilon}}\left(u_{\varepsilon, j}, \varphi\right)=\sum_{j=1}^{s^{\prime}} Q_{K_{\varepsilon} \cap V_{j}}\left(\widehat{u}_{\varepsilon, j}, \varphi\right)+\sum_{j=s^{\prime}+1}^{s} Q_{K_{\varepsilon}}\left(u_{\varepsilon, j}, \varphi\right) \\
& =\sum_{j=1}^{s^{\prime}} Q_{\left(K_{\varepsilon} \cap V_{j}\right) \backslash r_{j}^{-1}\left(K_{\varepsilon, j}\right)}\left(\widehat{u}_{\varepsilon, j}, \varphi\right)+\sum_{j=1}^{s^{\prime}} Q_{r_{j}^{-1}\left(K_{\varepsilon, j}\right)}\left(\widehat{u}_{\varepsilon, j}, \varphi\right)+\sum_{j=s^{\prime}+1}^{s} Q_{K_{\varepsilon}}\left(u_{\varepsilon, j}, \varphi\right) \\
& =\sum_{j=1}^{s^{\prime}} Q_{\left(K_{\varepsilon} \cap V_{j}\right) \backslash r_{j}^{-1}\left(K_{\varepsilon, j}\right)}\left(\widehat{u}_{\varepsilon, j}, \varphi\right)+\sum_{j=1}^{s^{\prime}} Q_{r_{j}^{-1}\left(K_{\varepsilon, j}\right)}\left(u_{\varepsilon, j}, \varphi\right)+\sum_{j=s^{\prime}+1}^{s} Q_{K_{\varepsilon}}\left(u_{\varepsilon, j}, \varphi\right) .
\end{aligned}
$$

From the end of the proof of Lemma 3.3 we infer that $\left\{\widehat{u}_{\varepsilon, j}\right\}_{0<\varepsilon \leq \varepsilon_{0}}$ is bounded in $H^{2}(\Omega)$.

Moreover $Q_{\left(K_{\varepsilon} \cap V_{j}\right) \backslash r_{j}^{-1}\left(K_{\varepsilon, j}\right)}(\varphi)=o(1)$. Therefore, as $\varepsilon \rightarrow 0$, we have

$$
\begin{aligned}
& \left|Q_{\left(K_{\varepsilon} \cap V_{j}\right) \backslash r_{j}^{-1}\left(K_{\varepsilon, j}\right)}\left(\widehat{u}_{\varepsilon, j}, \varphi\right)\right| \leq\left(Q_{\Omega}\left(\widehat{u}_{\varepsilon, j}\right)\right)^{1 / 2}\left(Q_{\left(K_{\varepsilon} \cap V_{j}\right) \backslash r_{j}^{-1}\left(K_{\varepsilon, j}\right)}(\varphi)\right)^{1 / 2}=o(1), \\
& \left|Q_{\left(K_{\varepsilon} \cap V_{j}\right) \backslash r_{j}^{-1}\left(K_{\varepsilon, j}\right)}\left(u_{\varepsilon, j}, \varphi\right)\right| \leq\left(Q_{\Omega_{\varepsilon}}\left(u_{\varepsilon}\right)\right)^{1 / 2}\left(Q_{\left(K_{\varepsilon} \cap V_{j}\right) \backslash r_{j}^{-1}\left(K_{\varepsilon, j}\right)}(\varphi)\right)^{1 / 2}=o(1) .
\end{aligned}
$$

Combining this with (83), we obtain

$$
\begin{aligned}
Q_{K_{\varepsilon}}\left(B_{\varepsilon} u_{\varepsilon}, \varphi\right) & =\sum_{j=1}^{s^{\prime}} Q_{\left(K_{\varepsilon} \cap V_{j}\right) \backslash r_{j}^{-1}\left(K_{\varepsilon, j}\right)}\left(u_{\varepsilon, j}, \varphi\right)+\sum_{j=1}^{s^{\prime}} Q_{r_{j}^{-1}\left(K_{\varepsilon, j}\right)}\left(u_{\varepsilon, j}, \varphi\right)+\sum_{j=s^{\prime}+1}^{s} Q_{K_{\varepsilon}}\left(u_{\varepsilon, j}, \varphi\right)+o(1) \\
& =Q_{K_{\varepsilon}}\left(u_{\varepsilon}, \varphi\right)+o(1) \quad \text { as } \varepsilon \rightarrow 0 .
\end{aligned}
$$


By (82) and (84) it follows

$$
Q_{K_{\varepsilon}}\left(u_{\varepsilon}, E_{\varepsilon} \varphi\right)=Q_{K_{\varepsilon}}\left(B_{\varepsilon} u_{\varepsilon}, \varphi\right)+o(1) \quad \text { as } \varepsilon \rightarrow 0 .
$$

Now, let us consider the second term on the right hand side of (81). We have

$$
\left|Q_{\Omega_{\varepsilon} \backslash K_{\varepsilon}}\left(u_{\varepsilon}, E_{\varepsilon} \varphi\right)\right| \leq\left(Q_{\Omega_{\varepsilon}}\left(u_{\varepsilon}\right)\right)^{1 / 2}\left(Q_{\Omega_{\varepsilon} \backslash K_{\varepsilon}}\left(E_{\varepsilon} \varphi\right)\right)^{1 / 2} .
$$

But, as $\varepsilon \rightarrow 0$, we have

$$
Q_{\Omega_{\varepsilon} \backslash K_{\varepsilon}}\left(E_{\varepsilon} \varphi\right) \leq s^{\prime} \sum_{j=1}^{s^{\prime}} Q_{\Omega_{\varepsilon} \backslash K_{\varepsilon}}\left(\tilde{\varphi}_{\varepsilon, j}\right) \leq s^{\prime} \sum_{j=1}^{s^{\prime}} Q_{\left(\Omega_{\varepsilon} \cap V_{j}\right) \backslash r_{j}^{-1}\left(K_{\varepsilon, j}\right)}\left(\tilde{\varphi}_{\varepsilon, j}\right)=o(1) .
$$

By (86) and (87) we arrive to $Q_{\Omega_{\varepsilon} \backslash K_{\varepsilon}}\left(u_{\varepsilon}, E_{\varepsilon} \varphi\right)=o(1)$ as $\varepsilon \rightarrow 0$.

Similarly we also have $Q_{\Omega \backslash K_{\varepsilon}}\left(B_{\varepsilon} u_{\varepsilon}, \varphi\right)=o(1)$ as $\varepsilon \rightarrow 0$. Inserting these two last asymptotic estimates and (85) into 81) we infer

$$
Q_{\Omega_{\varepsilon}}\left(u_{\varepsilon}, E_{\varepsilon} \varphi\right)=Q_{K_{\varepsilon}}\left(B_{\varepsilon} u_{\varepsilon}, \varphi\right)+Q_{\Omega \backslash K_{\varepsilon}}\left(B_{\varepsilon} u_{\varepsilon}, \varphi\right)+o(1)=Q_{\Omega}\left(B_{\varepsilon} u_{\varepsilon}, \varphi\right)+o(1) \quad \text { as } \varepsilon \rightarrow 0 .
$$

Since $B_{\varepsilon} u_{\varepsilon} \rightarrow \tilde{u}$ in $V(\Omega)$ then

$$
Q_{\Omega_{\varepsilon}}\left(u_{\varepsilon}, E_{\varepsilon} \varphi\right) \rightarrow Q_{\Omega}(\tilde{u}, \varphi) \quad \text { as } \varepsilon \rightarrow 0 .
$$

Step 4. The next purpose is to pass to the limit in the right hand side of (80).

First of all we observe that thanks to Lemma 3.1

$$
\begin{aligned}
& \left|\int_{\partial \Omega_{\varepsilon}}\left(w_{\varepsilon}\right)_{\nu}\left(E_{\varepsilon} \varphi\right)_{\nu} d S-\int_{\partial \Omega_{\varepsilon}}\left(E_{\varepsilon} w\right)_{\nu}\left(E_{\varepsilon} \varphi\right)_{\nu} d S\right| \\
& \quad \leq 2\left(d_{1}^{\varepsilon}\right)^{-1}\left\|w_{\varepsilon}-E_{\varepsilon} w\right\|_{V\left(\Omega_{\varepsilon}\right)} \cdot\left(\|\varphi\|_{V(\Omega)}+o(1)\right)=o(1),
\end{aligned}
$$

as $\varepsilon \rightarrow 0$. We claim that

$$
\int_{\partial \Omega_{\varepsilon}}\left(E_{\varepsilon} w\right)_{\nu}\left(E_{\varepsilon} \varphi\right)_{\nu} d S \rightarrow \int_{\partial \Omega} w_{\nu} \varphi_{\nu} d S \quad \text { as } \varepsilon \rightarrow 0
$$

In order to estimate $\int_{\partial \Omega_{\varepsilon}}\left(E_{\varepsilon} w\right)_{\nu}\left(E_{\varepsilon} \varphi\right)_{\nu} d S$ we proceed as follows:

$$
\begin{aligned}
& \int_{\partial \Omega_{\varepsilon}}\left(E_{\varepsilon} w\right)_{\nu}\left(E_{\varepsilon} \varphi\right)_{\nu} d S=\sum_{n, m=1}^{N} \int_{\partial \Omega_{\varepsilon}} \frac{\partial\left(E_{\varepsilon} w\right)}{\partial x_{n}} \nu_{\varepsilon, n} \frac{\partial\left(E_{\varepsilon} \varphi\right)}{\partial x_{m}} \nu_{\varepsilon, m} d S \\
& =\sum_{n, m=1}^{N} \sum_{i, j=1}^{s^{\prime}} \int_{\partial \Omega_{\varepsilon} \cap V_{i} \cap V_{j}} \frac{\partial\left(w_{i}\left(\Psi_{\varepsilon, i}(x)\right)\right)}{\partial x_{n}} \frac{\partial\left(\varphi_{j}\left(\Psi_{\varepsilon, j}(x)\right)\right)}{\partial x_{m}} \nu_{\varepsilon, n}(x) \nu_{\varepsilon, m}(x) d S \\
& =\sum_{n, m, k, l=1}^{N} \sum_{i, j=1}^{s^{\prime}} \int_{\Psi_{\varepsilon, j}\left(\partial \Omega_{\varepsilon} \cap V_{i} \cap V_{j}\right)} \frac{\partial w_{i}}{\partial y_{k}}\left(\Psi_{\varepsilon, i}\left(\Psi_{\varepsilon, j}^{-1}(y)\right)\right) \frac{\partial \varphi_{j}}{\partial y_{l}}(y) W_{\varepsilon, n, m, k, l, i, j}(y) d S .
\end{aligned}
$$

where we denoted by $\nu_{\varepsilon}=\left(\nu_{\varepsilon, 1}, \ldots, \nu_{\varepsilon, N}\right)$ the unit normal to $\partial \Omega_{\varepsilon}$ and we put (93)

$$
W_{\varepsilon, n, m, k, l, i, j}(y):=\nu_{\varepsilon, n}\left(\Psi_{\varepsilon, j}^{-1}(y)\right) \nu_{\varepsilon, m}\left(\Psi_{\varepsilon, j}^{-1}(y)\right) \frac{\partial\left(\Psi_{\varepsilon, i}\right)_{k}}{\partial x_{n}}\left(\Psi_{\varepsilon, j}^{-1}(y)\right) \frac{\partial\left(\Psi_{\varepsilon, j}\right)_{l}}{\partial x_{m}}\left(\Psi_{\varepsilon, j}^{-1}(y)\right) \frac{\sqrt{1+\left|\nabla_{x^{\prime}} g_{\varepsilon, j}\left(\Gamma_{j}(y)\right)\right|^{2}}}{\sqrt{1+\mid \nabla_{x^{\prime}} g_{j}\left(\Gamma_{j}(y)\right)^{2}}} .
$$

By (21) we deduce that the trivial extension of $W_{\varepsilon, n, m, k, l, i, j}$ to the whole $\partial \Omega$ converges almost everywhere to the function $\nu_{n} \nu_{m} \delta_{k n} \delta_{l m} \chi_{\partial \Omega \cap V_{i} \cap V_{j}}$ and it remains uniformly bounded as $\varepsilon \rightarrow 0$. Then, applying Lemma 3.7 (ii) to $w$, by (92) we obtain as $\varepsilon \rightarrow 0$

$$
\int_{\partial \Omega_{\varepsilon}}\left(E_{\varepsilon} w\right)_{\nu}\left(E_{\varepsilon} \varphi\right)_{\nu} d S \rightarrow \sum_{n, m=1}^{N} \sum_{i, j=1}^{s^{\prime}} \int_{\partial \Omega \cap V_{i} \cap V_{j}} \frac{\partial w_{i}}{\partial y_{n}} \frac{\partial \varphi_{j}}{\partial y_{m}} \nu_{n} \nu_{m} d S=\int_{\partial \Omega} w_{\nu} \varphi_{\nu}, d S .
$$


This completes the proof of (91). In turn, by (90)-(91), we obtain

$$
\int_{\partial \Omega_{\varepsilon}}\left(w_{\varepsilon}\right)_{\nu}\left(E_{\varepsilon} \varphi\right)_{\nu} d S \rightarrow \int_{\partial \Omega} w_{\nu} \varphi_{\nu} d S \quad \text { as } \varepsilon \rightarrow 0 .
$$

Step 5. In this last step we complete the proof of the lemma.

Inserting (89) and (94) into (80) we deduce that

$$
Q_{\Omega}(\tilde{u}, \varphi)=\int_{\partial \Omega} w_{\nu} \varphi_{\nu} d S \quad \text { for any } \varphi \in V(\Omega)
$$

On the other hand, also the function $u$ is a solution of the same variational problem and hence, by uniqueness of the solution of such a problem, we conclude that $\tilde{u}=u$. In particular this means that the weak limit $\tilde{u}$ does not depend on the choice of the sequence $\varepsilon \downarrow 0$, thus proving that the convergence $B_{\varepsilon} u_{\varepsilon} \rightarrow u$ does not occur only along a special sequence but as $\varepsilon \rightarrow 0$ in the usual sense. This completes the proof of the lemma.

Lemma 3.9. Let $\mathcal{A}$ be an atlas. Let $\left\{\Omega_{\varepsilon}\right\}_{0<\varepsilon \leq \varepsilon_{0}}$ be a family of domains of class $C^{1,1}(\mathcal{A})$ and $\Omega$ a domain of class $C^{1,1}(\mathcal{A})$. Assume the validity of condition (21). Let $w_{\varepsilon} \in V\left(\Omega_{\varepsilon}\right)$ with $0<\varepsilon \leq \varepsilon_{0}$, and $w \in V(\Omega)$ be such that $w_{\varepsilon} \stackrel{E}{\longrightarrow} w$. If we put $u_{\varepsilon}:=S_{\Delta, \varepsilon} w_{\varepsilon}$ and $u:=S_{\Delta}$ w or $u_{\varepsilon}:=S_{D^{2}, \varepsilon} w_{\varepsilon}$ and $u:=S_{D^{2}} w$ then $u_{\varepsilon} \stackrel{E}{\longrightarrow} u$. In particular this implies that $S_{\Delta, \varepsilon} \stackrel{E E}{\longrightarrow} S_{\Delta}$ and $S_{D^{2}, \varepsilon} \stackrel{E E}{\longrightarrow} S_{D^{2}}$ as $\varepsilon \rightarrow 0$ in the sense of Definition 2.2

Proof. We use the notation of (61), (62), (63) in order to treat the problems (2) and (5) simultaneously.

Let us consider

$$
\left\|u_{\varepsilon}-E_{\varepsilon} u\right\|_{V\left(\Omega_{\varepsilon}\right)}^{2}=\left\|u_{\varepsilon}\right\|_{V\left(\Omega_{\varepsilon}\right)}^{2}-2 Q_{\Omega_{\varepsilon}}\left(u_{\varepsilon}, E_{\varepsilon} u\right)+\left\|E_{\varepsilon} u\right\|_{V\left(\Omega_{\varepsilon}\right)}^{2} .
$$

Proceeding as in (88) and (89) we obtain

$$
Q_{\Omega_{\varepsilon}}\left(u_{\varepsilon}, E_{\varepsilon} u\right)=Q_{\Omega}\left(B_{\varepsilon} u_{\varepsilon}, u\right)+o(1) \rightarrow Q_{\Omega}(u, u) .
$$

The fact that $Q_{\Omega}\left(B_{\varepsilon} u_{\varepsilon}, u\right) \rightarrow Q_{\Omega}(u, u)$ is a consequence of the weak convergence $B_{\varepsilon} u_{\varepsilon} \rightarrow u$ in $V(\Omega)$ proved in Lemma 3.8. On the other hand by Lemma3.1 we have

$$
\left\|E_{\varepsilon} u\right\|_{V\left(\Omega_{\varepsilon}\right)}^{2} \rightarrow\|u\|_{V(\Omega)}^{2}=Q_{\Omega}(u, u) .
$$

It remains to prove that $\left\|u_{\varepsilon}\right\|_{V\left(\Omega_{\varepsilon}\right)}^{2} \rightarrow\|u\|_{V(\Omega)}^{2}$. We proceed as follows

$$
Q_{\Omega_{\varepsilon}}\left(u_{\varepsilon}, u_{\varepsilon}\right)=\int_{\partial \Omega_{\varepsilon}}\left(w_{\varepsilon}\right)_{\nu}\left(u_{\varepsilon}\right)_{\nu} d S=\int_{\partial \Omega_{\varepsilon}}\left(E_{\varepsilon} w\right)_{\nu}\left(u_{\varepsilon}\right)_{\nu} d S+o(1)
$$

where the second identity can be obtained by proceeding exactly as in 90) and exploiting the fact that $\left\|u_{\varepsilon}\right\|_{V\left(\Omega_{\varepsilon}\right)}=O(1)$ as $\varepsilon \rightarrow 0$ as we have shown in (79).

We claim that

$$
\int_{\partial \Omega_{\varepsilon}}\left(E_{\varepsilon} w\right)_{\nu}\left(u_{\varepsilon}\right)_{\nu} d S \rightarrow \int_{\partial \Omega} w_{\nu} u_{\nu} d S=Q_{\Omega}(u, u)
$$

Inserting (96)-(99) into (95) we obtain the proof of the lemma. It remains to prove (99).

In order to estimate $\int_{\partial \Omega_{\varepsilon}}\left(E_{\varepsilon} w\right)_{\nu}\left(u_{\varepsilon}\right)_{\nu} d S$ we proceed as follows:

$$
\begin{aligned}
& \int_{\partial \Omega_{\varepsilon}}\left(E_{\varepsilon} w\right)_{\nu}\left(u_{\varepsilon}\right)_{\nu} d S=\sum_{n, m=1}^{N} \int_{\partial \Omega_{\varepsilon}} \frac{\partial\left(E_{\varepsilon} w\right)}{\partial x_{n}} \nu_{\varepsilon, n} \frac{\partial u_{\varepsilon}}{\partial x_{m}} \nu_{\varepsilon, m} d S \\
& =\sum_{n, m=1}^{N} \sum_{i, j=1}^{s^{\prime}} \int_{\partial \Omega_{\varepsilon} \cap V_{i} \cap V_{j}} \frac{\partial\left(w_{i}\left(\Psi_{\varepsilon, i}(x)\right)\right)}{\partial x_{n}} \frac{\partial u_{\varepsilon, j}}{\partial x_{m}}(x) \nu_{\varepsilon, n}(x) \nu_{\varepsilon, m}(x) d S \\
& =\sum_{n, m, k, l=1}^{N} \sum_{i, j=1}^{s^{\prime}} \int_{\Psi_{\varepsilon, j}\left(\partial \Omega_{\varepsilon} \cap V_{i} \cap V_{j}\right)} \frac{\partial w_{i}}{\partial y_{k}}\left(\Psi_{\varepsilon, i}\left(\Psi_{\varepsilon, j}^{-1}(y)\right)\right) \frac{\partial \widehat{u}_{\varepsilon, j}}{\partial y_{l}}(y) W_{\varepsilon, n, m, k, l, i, j}(y) d S .
\end{aligned}
$$


with $\nu_{\varepsilon, n}, \nu_{\varepsilon, m}$ and $W_{\varepsilon, n, m, k, l, i, j}$ as in (93).

Applying Lemma 3.7 (ii) to $w$, applying (59) to $u_{\varepsilon}$, exploiting the fact that $B_{\varepsilon} u_{\varepsilon} \rightarrow u$ in $V(\Omega)$, and proceeding as at the end of the proof of Lemma3.8, as $\varepsilon \rightarrow 0$, we have

$$
\begin{aligned}
& \int_{\partial \Omega_{\varepsilon}}\left(E_{\varepsilon} w\right)_{\nu}\left(u_{\varepsilon}\right)_{\nu} d S=\sum_{n, m=1}^{N} \sum_{i, j=1}^{s^{\prime}} \int_{\partial \Omega \cap V_{i} \cap V_{j}} \frac{\partial w_{i}}{\partial y_{n}} \frac{\partial \widehat{u}_{\varepsilon, j}}{\partial y_{m}} \nu_{n} \nu_{m} d S+o(1) \\
& =\sum_{n, m=1}^{N} \sum_{i=1}^{s^{\prime}} \int_{\partial \Omega \cap V_{i}} \frac{\partial w_{i}}{\partial y_{n}} \frac{\partial\left(B_{\varepsilon} u_{\varepsilon}\right)}{\partial y_{m}} \nu_{n} \nu_{m} d S+o(1) \\
& =\sum_{n, m=1}^{N} \sum_{i=1}^{s^{\prime}} \int_{\partial \Omega \cap V_{i}} \frac{\partial w_{i}}{\partial y_{n}} \frac{\partial u}{\partial y_{m}} \nu_{n} \nu_{m} d S+o(1)=\int_{\partial \Omega} w_{\nu} u_{\nu} d S+o(1) .
\end{aligned}
$$

This completes the proof of 99] and of the lemma.

Lemma 3.10. Let $\mathcal{A}$ be an atlas. Let $\left\{\Omega_{\varepsilon}\right\}_{0<\varepsilon \leq \varepsilon_{0}}$ be a family of domains of class $C^{1,1}(\mathcal{A})$ and $\Omega$ a domain of class $C^{1,1}(\mathcal{A})$. Assume the validity of condition [21). Let $w_{\varepsilon} \in V\left(\Omega_{\varepsilon}\right)$ with $0<\varepsilon \leq \varepsilon_{0}$ be such that $\left\|w_{\varepsilon}\right\|_{V\left(\Omega_{\varepsilon}\right)}=1$. Then $\left\{S_{\Delta, \varepsilon} w_{\varepsilon}\right\}_{0<\varepsilon \leq \varepsilon_{0}}$ and $\left\{S_{D^{2}, \varepsilon} w_{\varepsilon}\right\}_{0<\varepsilon \leq \varepsilon_{0}}$ are precompact in the sense of Definition 2.3 In particular, by Definition 2.4 and Lemma 3.9 we have that $S_{\Delta, \varepsilon} \stackrel{C}{\longrightarrow} S_{\Delta}$ and $S_{D^{2}, \varepsilon} \stackrel{C}{\longrightarrow} S_{D^{2}}$ as $\varepsilon \rightarrow 0$.

Proof. According to the notations of Lemma 3.8 we put $u_{\varepsilon}:=S_{\Delta, \varepsilon} w_{\varepsilon}$ for problem (2) and $u_{\varepsilon}:=S_{D^{2}{ }_{,} \varepsilon} w_{\varepsilon}$ for problem (5). Exploiting the fact that $\left\|w_{\varepsilon}\right\|_{V\left(\Omega_{\varepsilon}\right)}$ is bounded we may proceed as in the proof of Lemma 3.8 and prove that $B_{\varepsilon} u_{\varepsilon} \rightarrow \widetilde{u}$ along a sequence, for some $\widetilde{u} \in V(\Omega)$. We divide the remaining part of the proof into four steps. We recall that as in the proof of Lemma 3.8 , for any $\varepsilon \in\left(0, \varepsilon_{0}\right]$ we have that $u_{\varepsilon}$ satisfies (80).

Step 1. In this step we pass to the limit in the right hand side of 800).

We put $h_{\varepsilon, n, i}:=\psi_{i} \frac{\partial w_{\varepsilon}}{\partial x_{n}}$ for $n=1, \ldots, N$ and $i=1, \ldots, s^{\prime}$, and we proceed as follows:

$$
\begin{aligned}
& \int_{\partial \Omega_{\varepsilon}}\left(w_{\varepsilon}\right)_{\nu}\left(E_{\varepsilon} \varphi\right)_{\nu} d S=\sum_{n, m=1}^{N} \int_{\partial \Omega_{\varepsilon}} \frac{\partial w_{\varepsilon}}{\partial x_{n}} \nu_{\varepsilon, n} \frac{\partial\left(E_{\varepsilon} \varphi\right)}{\partial x_{m}} \nu_{\varepsilon, m} d S \\
& =\sum_{n, m=1}^{N} \sum_{i, j=1}^{s^{\prime}} \int_{\partial \Omega_{\varepsilon} \cap V_{i} \cap V_{j}} h_{\varepsilon, n, i}(x) \frac{\partial\left(\varphi_{j}\left(\Psi_{\varepsilon, j}(x)\right)\right)}{\partial x_{m}} \nu_{\varepsilon, n}(x) \nu_{\varepsilon, m}(x) d S \\
& =\sum_{n, m, l=1}^{N} \sum_{i, j=1}^{s^{\prime}} \int_{\Psi_{\varepsilon, j}\left(\partial \Omega_{\varepsilon} \cap V_{i} \cap V_{j}\right)} h_{\varepsilon, n, i}\left(\Psi_{\varepsilon, j}^{-1}(y)\right) \frac{\partial \varphi_{j}}{\partial y_{l}}(y) W_{\varepsilon, n, m, l, j}(y) d S .
\end{aligned}
$$

where we denoted by $\nu_{\varepsilon}=\left(\nu_{\varepsilon, 1}, \ldots, \nu_{\varepsilon, N}\right)$ the unit normal to $\partial \Omega_{\varepsilon}$ and we put

$$
W_{\varepsilon, n, m, l, j}(y):=\nu_{\varepsilon, n}\left(\Psi_{\varepsilon, j}^{-1}(y)\right) \nu_{\varepsilon, m}\left(\Psi_{\varepsilon, j}^{-1}(y)\right) \frac{\partial\left(\Psi_{\varepsilon, j}\right)_{l}}{\partial x_{m}}\left(\Psi_{\varepsilon, j}^{-1}(y)\right) \frac{\sqrt{1+\left|\nabla_{x^{\prime}} g_{\varepsilon, j}\left(\Gamma_{j}(y)\right)\right|^{2}}}{\sqrt{1+\mid \nabla_{x^{\prime}} g_{j}\left(\left.\Gamma_{j}(y)\right|^{2}\right.}} .
$$

By (21) we deduce that the trivial extension of $W_{\varepsilon, n, m, l, j}$ to the whole $\partial \Omega$ converges almost everywhere to the function $\nu_{n} \nu_{m} \delta_{l m} \chi_{\partial \Omega \cap V_{i} \cap V_{j}}$ and it remains uniformly bounded as $\varepsilon \rightarrow 0$.

Now we observe that by Lemma 3.4 and Lemma 3.5, there exists a positive constant $C$ such that

$$
\left\|h_{\varepsilon, n, i}\right\|_{H^{1 / 2}\left(\partial \Omega_{\varepsilon}\right)}<C \quad \text { for any } \varepsilon \in\left(0, \varepsilon_{0}\right] .
$$

Hence, if we put $\omega_{\varepsilon, n, i}=h_{\varepsilon, n, i} \circ \Psi_{\varepsilon, i}^{-1}$, by [65), we also deduce that $\left\{\omega_{\varepsilon, n, i}\right\}_{0<\varepsilon \leq \varepsilon_{0}}$ is bounded in $H^{1 / 2}(\partial \Omega)$ and by the compact embedding $H^{1 / 2}(\partial \Omega) \subset L^{2}(\partial \Omega)$ we also have that $\left\{\omega_{\varepsilon, n, i}\right\}_{0<\varepsilon \leq \varepsilon_{0}}$ is precompact in $L^{2}(\partial \Omega)$. Then, along a sequence $\varepsilon_{k} \downarrow 0$ we may assume that $\omega_{\varepsilon_{k}, n, i} \rightarrow F_{n, i}$ in $L^{2}(\partial \Omega)$ as $k \rightarrow+\infty$. For simplicity, in the rest of the proof of the lemma we will omit the subindex $k$ and we simply write $\omega_{\varepsilon, n, i} \rightarrow F_{n, i}$ in $L^{2}(\partial \Omega)$ as $\varepsilon \rightarrow 0$.

But $\omega_{\varepsilon, n, i}\left(\Theta_{\varepsilon, i, j}(y)\right)=h_{\varepsilon, n, i}\left(\Psi_{\varepsilon, j}^{-1}(y)\right)$ for any $y \in \Psi_{\varepsilon, j}\left(\partial \Omega_{\varepsilon} \cap V_{i} \cap V_{j}\right)$. 
Then, applying Lemma3.7(ii) to $\omega_{\varepsilon, n, i}$, by (101) we obtain as $\varepsilon \rightarrow 0$

$$
\int_{\partial \Omega_{\varepsilon}}\left(w_{\varepsilon}\right)_{\nu}\left(E_{\varepsilon} \varphi\right)_{\nu} d S \rightarrow \sum_{n, m=1}^{N} \sum_{i, j=1}^{s^{\prime}} \int_{\partial \Omega \cap V_{i} \cap V_{j}} F_{n, i} \frac{\partial \varphi_{j}}{\partial y_{m}} \nu_{n} \nu_{m} d S=\int_{\partial \Omega}(F \cdot \nu) \varphi_{\nu} d S
$$

where we put $F=\left(\sum_{i=1}^{s^{\prime}} F_{1, i}, \ldots, \sum_{i=1}^{s^{\prime}} F_{N, i}\right)$.

Step 2. In this step we pass to the limit in the left hand side of 800 .

We can proceed exactly as in the proof of Step 3 in Lemma 3.8 since the only fact exploited there, is that the $V\left(\Omega_{\varepsilon}\right)$-norm of $u_{\varepsilon}$ can be estimated uniformly with respect to $\varepsilon$. In this way, one proves that $(89)$ holds true.

Inserting (89) and (102) into (80) we obtain

$$
Q_{\Omega}(\widetilde{u}, \varphi)=\int_{\partial \Omega}(F \cdot \nu) \varphi_{\nu} d S \quad \text { for any } \varphi \in V(\Omega) .
$$

Step 3. The purpose of this step is to pass to the limit in the right hand side of the following identity

$$
Q_{\Omega_{\varepsilon}}\left(u_{\varepsilon}, u_{\varepsilon}\right)=\int_{\partial \Omega_{\varepsilon}}\left(w_{\varepsilon}\right)_{\nu}\left(u_{\varepsilon}\right)_{\nu} d S .
$$

Similarly to (101) we can write

$$
\int_{\partial \Omega_{\varepsilon}}\left(w_{\varepsilon}\right)_{\nu}\left(u_{\varepsilon}\right)_{\nu} d S=\sum_{n, m, l=1}^{N} \sum_{i, j=1}^{s^{\prime}} \int_{\Psi_{\varepsilon, j}\left(\partial \Omega_{\varepsilon} \cap V_{i} \cap V_{j}\right)} h_{\varepsilon, n, i}\left(\Psi_{\varepsilon, j}^{-1}(y)\right) \frac{\partial \widehat{u}_{\varepsilon, j}}{\partial y_{l}}(y) W_{\varepsilon, n, m, l, j}(y) d S
$$

with $h_{\varepsilon, n, i}$ and $W_{\varepsilon, n, m, l, j}$ as in Step 1 , and $\widehat{u}_{\varepsilon, j}$ as in (43). By (59) we deduce that, passing to the limit along a subsequence of the sequence $\left\{\varepsilon_{k}\right\}$ introduced in Step 1 , for any $j \in\left\{1, \ldots, s^{\prime}\right\}$ there exists a function $U_{j} \in V(\Omega)$ such that $\widehat{u}_{\varepsilon, j} \rightarrow U_{j}$ in $V(\Omega)$. Using the same notations of Step 1, we simply write $\varepsilon \rightarrow 0$ to denote the convergence along a sequence. By [105, we then have as $\varepsilon \rightarrow 0$

$$
\int_{\partial \Omega_{\varepsilon}}\left(w_{\varepsilon}\right)_{\nu}\left(u_{\varepsilon}\right)_{\nu} d S \rightarrow \sum_{n, m, l=1}^{N} \sum_{i, j=1}^{s^{\prime}} \int_{\partial \Omega \cap V_{i} \cap V_{j}} F_{n, i} \frac{\partial U_{j}}{\partial y_{l}} \nu_{n} \nu_{m} d S
$$

Since $B_{\varepsilon} u_{\varepsilon}=\sum_{j=1}^{s^{\prime}} \widehat{u}_{\varepsilon, j}$ on $\partial \Omega$, from the uniqueness of the weak limit in $V(\Omega)$, one immediately obtains $\widetilde{u}=\sum_{j=1}^{s^{\prime}} U_{j}$ which inserted into (106) gives

$$
\int_{\partial \Omega_{\varepsilon}}\left(w_{\varepsilon}\right)_{\nu}\left(u_{\varepsilon}\right)_{\nu} d S \rightarrow \int_{\partial \Omega}(F \cdot \nu) \widetilde{u}_{\nu} d S
$$

Step 4. In this step we conclude the proof of the lemma.

Choosing $\varphi=\widetilde{u}$ in (103) and combining this with 104), (107), we obtain as $\varepsilon \rightarrow 0$ along a proper sequence $\varepsilon_{k} \downarrow 0$,

$$
\left\|u_{\varepsilon}\right\|_{V\left(\Omega_{\varepsilon}\right)}^{2}=Q_{\Omega_{\varepsilon}}\left(u_{\varepsilon}, u_{\varepsilon}\right)=\int_{\partial \Omega_{\varepsilon}}\left(w_{\varepsilon}\right)_{\nu}\left(u_{\varepsilon}\right)_{\nu} d S \rightarrow \int_{\partial \Omega}(F \cdot \nu) \widetilde{u}_{\nu} d S=Q_{\Omega}(\widetilde{u}, \widetilde{u})=\|\widetilde{u}\|_{V(\Omega)}^{2} .
$$

On the other hand, by (80), (102), (103) with $\varphi=\widetilde{u}$, we obtain as $\varepsilon \rightarrow 0$ along the same sequence converging to zero,

$$
Q_{\Omega_{\varepsilon}}\left(u_{\varepsilon}, E_{\varepsilon} \widetilde{u}\right) \rightarrow Q_{\Omega}(\widetilde{u}, \widetilde{u})=\|\widetilde{u}\|_{V(\Omega)}^{2} .
$$

Combining (108) and (109) with Lemma 3.1(ii), we obtain

$$
\left\|u_{\varepsilon}-E_{\varepsilon} \widetilde{u}\right\|_{V\left(\Omega_{\varepsilon}\right)}^{2}=\left\|u_{\varepsilon}\right\|_{V\left(\Omega_{\varepsilon}\right)}^{2}-2 Q_{\Omega_{\varepsilon}}\left(u_{\varepsilon}, E_{\varepsilon} \widetilde{u}\right)+\left\|E_{\varepsilon} \widetilde{u}\right\|_{V\left(\Omega_{\varepsilon}\right)}^{2} \rightarrow 0 .
$$

This proves that along a sequence we have $u_{\varepsilon} \stackrel{E}{\longrightarrow} \widetilde{u}$ as $\varepsilon \rightarrow 0$ or equivalently that $\left\{S_{\Delta, \varepsilon} w_{\varepsilon}\right\}_{0<\varepsilon \leq \varepsilon_{0}}$ and $\left\{S_{D^{2}{ }_{\varepsilon} \varepsilon} w_{\varepsilon}\right\}_{0<\varepsilon \leq \varepsilon_{0}}$ are precompact in the sense of Definition 2.3. The proof of the lemma now follows from Lemma 3.9 and Definition 2.4 
As a bypass product of a number of results proved in this section, we have the following proposition which we believe has its own interest since it clarifies even more the meaning of $E$-convergence with respect to the operators $E_{\varepsilon}$ used above.

Proposition 3.11. Let $\mathcal{A}$ be an atlas. Let $\left\{\Omega_{\varepsilon}\right\}_{0<\varepsilon \leq \varepsilon_{0}}$ be a family of domains of class $C^{1,1}(\mathcal{A}), \Omega$ a domain of class $C^{1,1}(\mathcal{A})$ and for any $\varepsilon \in\left(0, \varepsilon_{0}\right]$ let $E_{\varepsilon}$ be the map defined in (26). Assume the validity of condition (21). If $u_{\varepsilon} \in V\left(\Omega_{\varepsilon}\right), u \in V(\Omega)$ is such that $u_{\varepsilon} \stackrel{E}{\longrightarrow} u$ as $\varepsilon \rightarrow 0$ then

$$
\left\|u_{\varepsilon}-u\right\|_{H^{2}\left(\Omega_{\varepsilon} \cap \Omega\right)} \rightarrow 0 \text {, and }\left\|\nabla u_{\varepsilon}-E_{\varepsilon} \nabla u\right\|_{L^{2}\left(\partial \Omega_{\varepsilon}\right)} \rightarrow 0,
$$

as $\varepsilon \rightarrow 0$.

Proof. Let $K_{\varepsilon} \subset \Omega_{\varepsilon} \cap \Omega$ be as in the proof of Lemma3.8. Recall that $E_{\varepsilon} u=u$ on $K_{\varepsilon}$. In order to prove the first limit in (3.11) it suffices to note that by Lemma 3.4 and the proof of Lemma 3.1 we have

$$
\begin{aligned}
\left\|u_{\varepsilon}-u\right\|_{H^{2}\left(\Omega_{\varepsilon} \cap \Omega\right)} & \leq\left\|u_{\varepsilon}-E_{\varepsilon} u\right\|_{H^{2}\left(\Omega_{\varepsilon} \cap \Omega\right)}+\left\|E_{\varepsilon} u-u\right\|_{H^{2}\left(\Omega_{\varepsilon} \cap \Omega\right)} \\
& \leq\left\|u_{\varepsilon}-E_{\varepsilon} u\right\|_{H^{2}\left(\Omega_{\varepsilon}\right)}+\left\|E_{\varepsilon} u-u\right\|_{H^{2}\left(\left(\Omega_{\varepsilon} \cap \Omega\right) \backslash K_{\varepsilon}\right)} \\
& \leq C\left\|u_{\varepsilon}-E_{\varepsilon} u\right\|_{V\left(\Omega_{\varepsilon}\right)}+\left\|E_{\varepsilon} u-u\right\|_{H^{2}\left(\left(\Omega_{\varepsilon} \cap \Omega\right) \backslash K_{\varepsilon}\right)} \\
& =C\left\|u_{\varepsilon}-E_{\varepsilon} u\right\|_{V\left(\Omega_{\varepsilon}\right)}+o(1) .
\end{aligned}
$$

In order to prove the second limit in 3.11) we note that by Lemma 3.5 the trace constant can be taken uniform with the respect to $\varepsilon$ hence $\left\|\nabla u_{\varepsilon}-\nabla\left(E_{\varepsilon} u\right)\right\|_{L^{2}\left(\partial \Omega_{\varepsilon}\right)} \leq C\left\|u_{\varepsilon}-E_{\varepsilon} u\right\|_{H^{2}\left(\Omega_{\varepsilon}\right)}=o(1)$. Finally, by standard computations one can see that $\left\|\nabla u_{\varepsilon}-E_{\varepsilon} \nabla u\right\|_{L^{2}\left(\partial \Omega_{\varepsilon}\right)}=\left\|\nabla u_{\varepsilon}-\nabla\left(E_{\varepsilon} u\right)\right\|_{L^{2}\left(\partial \Omega_{\varepsilon}\right)}+o(1)$.

\section{OPTIMALITY OF CONDITION (21): DEGENERATIONS AND STRANGE TERMS}

We plan to discuss the optimality of condition (21). We ask whether spectral stability is still verified if we remove condition (21) from the statement of Theorem 3.2. In order to give an answer to this question we assume particular conditions on the domain $\Omega$ and on its perturbations $\Omega_{\varepsilon}$. We follow closely the approach introduced in [4]. We assume that $\Omega$ is in the form $\Omega=W \times(-1,0)$ where $W$ is a cuboid or a bounded domain in $\mathbb{R}^{N-1}$ of class $C^{1,1}$. We assume that the perturbed domain $\Omega_{\varepsilon}$ is given by

$$
\Omega_{\varepsilon}=\left\{\left(x^{\prime}, x_{N}\right): x^{\prime} \in W,-1<x_{N}<g_{\varepsilon}\left(x^{\prime}\right)\right\}
$$

where $g_{\varepsilon}\left(x^{\prime}\right)=\varepsilon^{\alpha} b\left(x^{\prime} / \varepsilon\right)$ for any $x^{\prime} \in W$ and $b: \mathbb{R}^{N-1} \rightarrow[0,+\infty)$ is a $Y$-periodic function where $Y=\left(-\frac{1}{2}, \frac{1}{2}\right)^{N-1}$ is the unit cell in $\mathbb{R}^{N-1}$.

We denote by $\Gamma_{\varepsilon}$ and $\Gamma$ the sets

$$
\Gamma_{\varepsilon}:=\left\{\left(x^{\prime}, g_{\varepsilon}\left(x^{\prime}\right)\right): x^{\prime} \in W\right\} \quad \text { and } \quad \Gamma:=W \times\{0\} .
$$

In this case, condition (21) can be used provided $\alpha>3 / 2$. In fact, we have the following straightforward application of Theorem 3.2 in the simplified form discussed in Remark 5

Corollary 4.1. Suppose that the function $b$ defined above belongs to $C^{1,1}\left(\mathbb{R}^{N-1}\right)$. Let $\Omega$ and $\Omega_{\varepsilon}$ be as above. Let $S_{\Delta, \varepsilon}, S_{\Delta}$ be the Steklov operators for (2) in $\Omega_{\varepsilon}$ and $\Omega$ respectively, and let $S_{D^{2}, \varepsilon}, S_{D^{2}}$ be the Steklov operators for (5) in $\Omega_{\varepsilon}$ and $\Omega$ respectively, see Subsection 3.1 If $\alpha>3 / 2$ then $S_{\Delta, \varepsilon} \stackrel{C}{\longrightarrow} S_{\Delta}$ and $S_{D^{2}, \varepsilon} \stackrel{C}{\longrightarrow} S_{D^{2}}$ as $\varepsilon \rightarrow 0$.

Proof. For fixed $\tilde{\alpha} \in] 3 / 2, \alpha$, we set $\kappa_{\varepsilon}=\varepsilon^{2 \tilde{\alpha} / 3}$ and we note that condition (21) is satisfied with $g_{\varepsilon, j}, g_{j}$ replaced by $g_{\varepsilon}, g \equiv 0$ respectively. The proof follows by Theorem 3.2 (actually, by the simplified version of it concerning the case of domains defined as subgraphs of functions defined in one single cuboid, see Remark 5).

We shall see that Corollary 4.1 no longer holds for problem (5) if $\alpha \leq 3 / 2$. The cases $\alpha<3 / 2$ and $\alpha=3 / 2$ are considerably different. In the first case we have degeneration to Dirichlet boundary conditions, in the second case we have the appearance of a strange term in the limiting boundary conditions. We discuss the two cases in the following subsections. 
4.1. Case $\alpha<3 / 2$ : degeneration. In this case it appears that the energy spaces $V\left(\Omega_{\varepsilon}\right)=H^{2}\left(\Omega_{\varepsilon}\right) \cap$ $H_{0}^{1}\left(\Omega_{\varepsilon}\right)$ degenerate as $\varepsilon \rightarrow 0$ to a suitable energy space which encodes Dirichlet boundary conditions on $\Gamma$. Namely, let us define the space

$$
H_{0, \Gamma}^{2}(\Omega):=\left\{u \in H^{2}(\Omega) \cap H_{0}^{1}(\Omega): u_{\nu}=0 \text { on } \Gamma\right\} .
$$

According to Section 3.1, we define $T_{D^{2}, \Gamma}: H_{0, \Gamma}^{2}(\Omega) \rightarrow\left(H_{0, \Gamma}^{2}(\Omega)\right)^{\prime}$ by

$$
\left(H_{0, \Gamma}^{2}(\Omega)\right)^{\prime}\left\langle T_{D^{2}, \Gamma} u, v\right\rangle_{H_{0, \Gamma}^{2}(\Omega)}:=\int_{\Omega} D^{2} u: D^{2} v d x \quad \text { for any } u, v \in H_{0, \Gamma}^{2}(\Omega) .
$$

We also define the operator $J_{\Gamma}: H_{0, \Gamma}^{2}(\Omega) \rightarrow\left(H_{0, \Gamma}^{2}(\Omega)\right)^{\prime}$ by

$$
\left(H_{0, \Gamma}^{2}(\Omega)\right)^{\prime}\left\langle J_{\Gamma} u, v\right\rangle_{H_{0, \Gamma}^{2}(\Omega)}:=\int_{\partial \Omega \backslash \Gamma} u_{\nu} v_{\nu} d S \quad \text { for any } u, v \in H_{0, \Gamma}^{2}(\Omega) .
$$

Finally we define $S_{D^{2}, \Gamma}: H_{0, \Gamma}^{2}(\Omega) \rightarrow H_{0, \Gamma}^{2}(\Omega)$ as $S_{D^{2}, \Gamma}:=T_{D^{2}, \Gamma}^{-1} \circ J_{\Gamma}$.

We shall prove that $S_{D^{2}, \varepsilon}$ converges compactly to $S_{D^{2}, \Gamma}$ as $\varepsilon \rightarrow 0$. To do so we need to clarify which family of operators $E_{\varepsilon}: H_{0, \Gamma}^{2}(\Omega) \rightarrow V\left(\Omega_{\varepsilon}\right)$ we consider. In the case $\alpha<3 / 2$ we can no longer use the operators $E_{\varepsilon}$ defined in Subsection 3.2 However, here it is possible to consider a much simpler family of operators given by the extension-by-zero operators.

Let us define the extension-by-zero operator $\mathcal{E}_{0}: H_{0, \Gamma}^{2}(\Omega) \rightarrow V\left(\Omega_{\varepsilon}\right)$ which maps any function $u \in$ $H_{0, \Gamma}^{2}(\Omega)$ to $\mathcal{E}_{0} u$ where $\mathcal{E}_{0} u: W \times(-1,+\infty) \rightarrow \mathbb{R}$ coincides with $u$ over $\Omega$ and vanishes in $W \times[0,+\infty)$. We observe that $\mathcal{E}_{0}$ is well-defined as an operator from $H_{0, \Gamma}^{2}(\Omega)$ to $V\left(\Omega_{\varepsilon}\right)$ since for any $u \in H_{0, \Gamma}^{2}(\Omega)$ we have $\mathcal{E}_{0} u \in H^{2}(W \times(-1,+\infty))$ being $u=u_{\nu}=0$ on $\Gamma$ and hence its restriction to $\Omega_{\varepsilon}$ belongs indeed to $V\left(\Omega_{\varepsilon}\right)$.

Since we are dealing with problem (5), the space $V\left(\Omega_{\varepsilon}\right)$ and the space $H_{0, \Gamma}^{2}(\Omega)$ will be endowed with the second scalar product in (15), i.e.

$$
\begin{aligned}
& \|u\|_{V\left(\Omega_{\varepsilon}\right)}:=\left(\int_{\Omega_{\varepsilon}}\left|D^{2} u\right|^{2} d x\right)^{1 / 2} \quad \text { for any } u \in V\left(\Omega_{\varepsilon}\right), \\
& \|u\|_{H_{0, \Gamma}^{2}(\Omega)}:=\left(\int_{\Omega}\left|D^{2} u\right|^{2} d x\right)^{1 / 2} \quad \text { for any } u \in H_{0, \Gamma}^{2}(\Omega) .
\end{aligned}
$$

According to Definitions 2.1 2.4, the following notions of convergence are well defined

$$
u_{\varepsilon} \stackrel{E}{\longrightarrow} u, \quad B_{\varepsilon} \stackrel{E E}{\longrightarrow} B_{0}, \quad B_{\varepsilon} \stackrel{C}{\longrightarrow} B_{0}
$$

once we put $\mathcal{H}_{\varepsilon}=V\left(\Omega_{\varepsilon}\right), \mathcal{H}_{0}=H_{0, \Gamma}^{2}(\Omega)$ and $E_{\varepsilon}=\mathcal{E}_{0}$. We observe that property (11) is trivially satisfied.

Then the following result holds

Theorem 4.1. Suppose that the function $b$ defined above belongs to $C^{1,1}\left(\mathbb{R}^{N-1}\right)$ and is not constant. Let $\Omega$ and $\Omega_{\varepsilon}$ be as above. Let $S_{D^{2}, \varepsilon}, S_{D^{2}}$ be the Steklov operators for $(5)$ in $\Omega_{\varepsilon}$ and $\Omega$ respectively, see Section 3.1 Finally, let $S_{D^{2}, \Gamma}: H_{0, \Gamma}^{2}(\Omega) \rightarrow H_{0, \Gamma}^{2}(\Omega)$ be as above. If $\alpha \in\left[1, \frac{3}{2}\right)$ then $S_{D^{2}, \varepsilon} \stackrel{C}{\longrightarrow} S_{D^{2}, \Gamma}$ as $\varepsilon \rightarrow 0$ and in particular the eigenvalues of (5) in $\Omega_{\varepsilon}$ converge, as $\varepsilon \rightarrow 0$, to the eigenvalues of the following problem

$$
\begin{cases}\Delta^{2} u=0, & \text { in } \Omega, \\ u=0, & \text { on } \partial \Omega, \\ u_{\nu}=0, & \text { on } \Gamma, \\ \Delta u-K(x) u_{\nu}-\delta u_{\nu}=0, & \text { on } \partial \Omega \backslash \Gamma .\end{cases}
$$

The proof of Theorem 4.1 is based on a number of technical results. First of all, we prove that the $V\left(\Omega_{\varepsilon}\right)$ norm is uniformly equivalent to the $H^{2}\left(\Omega_{\varepsilon}\right)$-norm.

Lemma 4.2. Suppose that all the assumptions of Theorem 4.1 are satisfied. Then there exist $\varepsilon_{0}>0$ and $C>0$ such that 
(i) for any $u \in V\left(\Omega_{\varepsilon}\right)$ and $\varepsilon \in\left(0, \varepsilon_{0}\right]$ we have

$$
\|u\|_{H^{2}\left(\Omega_{\varepsilon}\right)} \leq C\|u\|_{V\left(\Omega_{\varepsilon}\right)}
$$

(ii) for any $v \in H^{1}\left(\Omega_{\varepsilon}\right)$ and $\varepsilon \in\left(0, \varepsilon_{0}\right]$ we have

$$
\|v\|_{L^{2}\left(\partial \Omega_{\varepsilon}\right)} \leq C\|v\|_{H^{1}\left(\Omega_{\varepsilon}\right)} .
$$

Proof. The proof of (i) is a consequence of Lemma 2.7, see also (45). The proof of (ii) follows from direct computation exploiting the fact that under the assumptions of Theorem 4.1, the first order derivatives of $g_{\varepsilon}$ remain uniformly bounded as $\varepsilon \rightarrow 0$.

As an immediate consequence of Lemma 4.2 we obtain the following uniform estimate for the first eigenvalue of (5).

Lemma 4.3. Suppose that all the assumptions of Theorem 4.1 are satisfied. Then

$$
\liminf _{\varepsilon \rightarrow 0} \delta_{1}^{\varepsilon}>0
$$

where $\delta_{1}^{\varepsilon}$ is defined by (18) with $\Omega_{\varepsilon}$ in place of $\Omega$.

Proof. We denote by $C$ a positive constant independent of $\varepsilon$ and $u$ which may vary from line to line. Let $u \in V\left(\Omega_{\varepsilon}\right)$ and for $i \in\{1, \ldots, N\}$ let us apply Lemma 4.2 to $\frac{\partial u}{\partial x_{i}}$ :

$$
\left\|\frac{\partial u}{\partial x_{i}}\right\|_{L^{2}\left(\partial \Omega_{\varepsilon}\right)} \leq C\left\|\frac{\partial u}{\partial x_{i}}\right\|_{H^{1}\left(\Omega_{\varepsilon}\right)} \leq C\|u\|_{H^{2}\left(\Omega_{\varepsilon}\right)} \leq C\|u\|_{V\left(\Omega_{\varepsilon}\right)} \quad \text { for any } u \in V\left(\Omega_{\varepsilon}\right) \text { and } \varepsilon \in\left(0, \varepsilon_{0}\right] \text {. }
$$

Thus

$$
\int_{\partial \Omega_{\varepsilon}} u_{\nu}^{2} d S \leq C \int_{\Omega_{\varepsilon}}\left|D^{2} u\right|^{2} d x \quad \text { for any } u \in V\left(\Omega_{\varepsilon}\right) \text { and } \varepsilon \in\left(0, \varepsilon_{0}\right] .
$$

The proof of the lemma then follows from (18).

Next we prove that the family of operators $\left\{S_{D^{2}, \varepsilon}\right\} E$-converges to $S_{D^{2}, \Gamma}$ as $\varepsilon \rightarrow 0$.

Lemma 4.4. Suppose that all the assumptions of Theorem 4.1 are satisfied. Then $S_{D^{2}, \varepsilon} \stackrel{E E}{\longrightarrow} S_{D^{2}, \Gamma}$ as $\varepsilon \rightarrow 0$.

Proof. Let $w_{\varepsilon} \in V\left(\Omega_{\varepsilon}\right)$ and $w \in H_{0, \Gamma}^{2}(\Omega)$ be such that $w_{\varepsilon} \stackrel{E}{\longrightarrow} w$ as $\varepsilon \rightarrow 0$. Put $u_{\varepsilon}:=S_{D^{2}, \varepsilon} w_{\varepsilon}$. We claim that $\left\|u_{\varepsilon}\right\|_{V\left(\Omega_{\varepsilon}\right)}$ remains bounded as $\varepsilon \rightarrow 0$. Using the same argument as in (78) we have $\left\|u_{\varepsilon}\right\|_{V\left(\Omega_{\varepsilon}\right)}^{2} \leq$ $\left(\delta_{1}^{\varepsilon}\right)^{-1}\left\|w_{\varepsilon}\right\|_{V\left(\Omega_{\varepsilon}\right)}\left\|u_{\varepsilon}\right\|_{V\left(\Omega_{\varepsilon}\right)}$ and this together with Lemma 4.3 proves the claim being $\left\|w_{\varepsilon}\right\|_{V\left(\Omega_{\varepsilon}\right)}$ bounded as $\varepsilon \rightarrow 0$ as a consequence of the fact that $w_{\varepsilon} \stackrel{E}{\longrightarrow} w$.

In particular by Lemma 4.2 (i) we also have that $\left\{\left(u_{\varepsilon}\right)_{\mid \Omega}\right\}_{0<\varepsilon \leq \varepsilon_{0}}$ is bounded in $H^{2}(\Omega)$ as $\varepsilon \rightarrow 0$ and hence along a sequence we have that there exists $u \in H^{2}(\Omega)$, possibly depending on the choice of the sequence, such that $\left(u_{\varepsilon}\right)_{\mid \Omega} \rightarrow u$ in $H^{2}(\Omega)$ as $\varepsilon \rightarrow 0$.

By taking the trivial extension of $u_{\varepsilon}$ to the whole $\mathbb{R}^{N}$, one sees that it is weakly convergent in $H^{1}\left(\mathbb{R}^{N}\right)$ and pointwise convergent almost everywhere (up to a subsequence) to a function belonging to $H^{1}\left(\mathbb{R}^{N}\right)$, whose restriction to $\Omega$ coincides with $u$. This shows that $u \in H_{0}^{1}(\Omega)$.

We claim that $u_{\nu}=\frac{\partial u}{\partial x_{N}}=0$ on $\Gamma$ thus showing that $u \in H_{0, \Gamma}^{2}(\Omega)$. In order to prove the claim we apply [18, Lemma 4.3] or more precisely its extension to the $N$-dimensional case. Indeed, for any $i \in$ $\{1, \ldots, N-1\}$, proceeding as in proof of Theorem 7.4 in [4], we define the family of vector fields depending on the parameter $\varepsilon$

$$
V_{\varepsilon}^{(i)}=\left(0, \ldots, 0,-\frac{\partial u_{\varepsilon}}{\partial x_{N}}, 0, \ldots, 0, \frac{\partial u_{\varepsilon}}{\partial x_{i}}\right)
$$

where the only two nontrivial components are the $i$-th and $N$-th ones. Since $u_{\varepsilon}\left(x^{\prime}, g_{\varepsilon}\left(x^{\prime}\right)\right)=0$ for any $x^{\prime} \in W$ it follows that $V_{\varepsilon}^{(i)} \cdot \nu=0$ on $\Gamma_{\varepsilon}$ and hence, since $\alpha<\frac{3}{2}$, we can apply [18, Lemma 4.3 (i)] to this vector field and obtain

$$
-\frac{\partial u}{\partial x_{N}}\left(x^{\prime}, 0\right) \frac{\partial b}{\partial y_{i}}\left(y^{\prime}\right)=0 \quad \text { for any } x^{\prime} \in W \text { and } y^{\prime} \in \mathbb{R}^{N-1} .
$$


Since $b$ is not constant we deduce that $\frac{\partial u}{\partial x_{N}} \equiv 0$ in $\Gamma$ thus proving the claim.

We now prove that $u$ does not depend on the sequence converging to zero. Letting $\varphi \in H_{0, \Gamma}^{2}(\Omega)$ we obtain

$$
\int_{\Omega} D^{2} u_{\varepsilon}: D^{2} \varphi d x=\int_{\Omega_{\varepsilon}} D^{2} u_{\varepsilon}: D^{2}\left(\mathcal{E}_{0} \varphi\right) d x=\int_{\partial \Omega_{\varepsilon}}\left(w_{\varepsilon}\right)_{\nu}\left(\mathcal{E}_{0} \varphi\right)_{\nu} d S=\int_{\partial \Omega \backslash \Gamma}\left(w_{\varepsilon}\right)_{\nu} \varphi_{\nu} d S .
$$

From the weak convergence $\left(u_{\varepsilon}\right)_{\mid \Omega} \rightarrow u$ in $H^{2}(\Omega)$, boundedness of $\left\{\left(w_{\varepsilon}\right)_{\mid \Omega}\right\}_{0<\varepsilon \leq \varepsilon_{0}}$ in $H^{2}(\Omega)$ as $\varepsilon \rightarrow 0$, and compactness of the trace map, we can pass to the limit in (114) as $\varepsilon \rightarrow 0$ along a sequence and obtain

$$
\int_{\Omega} D^{2} u: D^{2} \varphi d x=\int_{\partial \Omega \backslash \Gamma} w_{\nu} \varphi_{\nu} d S \quad \text { for any } \varphi \in H_{0, \Gamma}^{2}(\Omega) .
$$

We observe that the first function appearing in the integral at the right hand side of (115) is exactly $w$ since, by Lemma $4.2(\mathrm{i}), w_{\varepsilon} \stackrel{E}{\longrightarrow} w$ implies $\left(w_{\varepsilon}\right)_{\mid \Omega} \rightarrow w$ in $H^{2}(\Omega)$.

The variational identity (115) proves that $u \in H_{0, \Gamma}^{2}(\Omega)$ is a weak solution of the problem

$$
\begin{cases}\Delta^{2} u=0, & \text { in } \Omega, \\ u=0, & \text { on } \partial \Omega, \\ u_{\nu}=0, & \text { on } \Gamma, \\ \Delta u-K(x) u_{\nu}=w_{\nu}, & \text { on } \partial \Omega \backslash \Gamma .\end{cases}
$$

Since it is easy to check that this problem admits a unique solution, we deduce that $u$ depends only on $w$ and not on the sequence converging to zero. Moreover, we also have that $u=S_{D^{2}, \Gamma} w$.

Then we prove that $u_{\varepsilon} \stackrel{E}{\longrightarrow} u$. Since $\mathcal{E}_{0} u$ vanishes outside $\Omega$ and $\left(u_{\varepsilon}\right)_{\mid \Omega} \rightarrow u$ in $H^{2}(\Omega)$, we have

$$
\begin{aligned}
& \| u_{\varepsilon}- \mathcal{E}_{0} u \|_{V\left(\Omega_{\varepsilon}\right)}^{2}=\int_{\Omega_{\varepsilon}}\left|D^{2} u_{\varepsilon}\right|^{2} d x-2 \int_{\Omega} D^{2} u_{\varepsilon}: D^{2} u d x+\int_{\Omega}\left|D^{2} u\right|^{2} d x \\
&=\int_{\Omega_{\varepsilon}}\left|D^{2} u_{\varepsilon}\right|^{2} d x-\int_{\Omega}\left|D^{2} u\right|^{2} d x+o(1) .
\end{aligned}
$$

Now we observe that

$$
\int_{\Omega_{\varepsilon}}\left|D^{2} u_{\varepsilon}\right|^{2} d x=\int_{\partial \Omega_{\varepsilon}}\left(w_{\varepsilon}\right)_{\nu}\left(u_{\varepsilon}\right)_{\nu} d S
$$

We prove that

$$
\int_{\partial \Omega_{\varepsilon}}\left(w_{\varepsilon}\right)_{\nu}\left(u_{\varepsilon}\right)_{\nu} d S \rightarrow \int_{\partial \Omega} w_{\nu} u_{\nu} d S=\int_{\partial \Omega \backslash \Gamma} w_{\nu} u_{\nu} d S
$$

Indeed we have

$$
\begin{aligned}
& \left|\int_{\partial \Omega_{\varepsilon}}\left(w_{\varepsilon}\right)_{\nu}\left(u_{\varepsilon}\right)_{\nu} d S-\int_{\partial \Omega} w_{\nu} u_{\nu} d S\right| \\
& \quad \leq\left|\int_{\partial \Omega_{\varepsilon}}\left(w_{\varepsilon}-\mathcal{E}_{0} w\right)_{\nu}\left(u_{\varepsilon}\right)_{\nu} d S\right|+\left|\int_{\partial \Omega_{\varepsilon}}\left(\mathcal{E}_{0} w\right)_{\nu}\left(u_{\varepsilon}\right)_{\nu} d S-\int_{\partial \Omega} w_{\nu} u_{\nu} d S\right| \\
& \quad \leq\left(\delta_{1}^{\varepsilon}\right)^{-1}\left\|w_{\varepsilon}-\mathcal{E}_{0} w\right\|_{V\left(\Omega_{\varepsilon}\right)}\left\|u_{\varepsilon}\right\|_{V\left(\Omega_{\varepsilon}\right)}+\left|\int_{\partial \Omega} w_{\nu}\left(u_{\varepsilon}\right)_{\nu} d S-\int_{\partial \Omega} w_{\nu} u_{\nu} d S\right|=o(1) \quad \text { as } \varepsilon \rightarrow 0
\end{aligned}
$$

since $\left(\delta_{1}^{\varepsilon}\right)^{-1}$ is bounded as $\varepsilon \rightarrow 0$ in view of Lemma 4.3. $\left\|u_{\varepsilon}\right\|_{V\left(\Omega_{\varepsilon}\right)}$ is bounded as $\varepsilon \rightarrow 0$ as already observed, $\left\|w_{\varepsilon}-\mathcal{E}_{0} u\right\|_{V\left(\Omega_{\varepsilon}\right)} \rightarrow 0$ as $\varepsilon \rightarrow 0$ since $w_{\varepsilon} \stackrel{E}{\longrightarrow} w$ and $\left(u_{\varepsilon}\right)_{\nu} \rightarrow u_{\nu}$ in $L^{2}(\partial \Omega)$ since $\left(u_{\varepsilon}\right)_{\mid \Omega} \rightarrow u$ in $H^{2}(\Omega)$ as $\varepsilon \rightarrow 0$. This proves the validity of (119).

Finally we observe that, by (115), we also have

$$
\int_{\Omega}\left|D^{2} u\right|^{2} d x=\int_{\partial \Omega \backslash \Gamma} w_{\nu} u_{\nu} d S
$$


Combining (118), (119) and (120) we infer that $\int_{\Omega_{\varepsilon}}\left|D^{2} u_{\varepsilon}\right|^{2} d x \rightarrow \int_{\Omega}\left|D^{2} u\right|^{2} d x$ as $\varepsilon \rightarrow 0$ which combined with (117) proves that $u_{\varepsilon} \stackrel{E}{\longrightarrow} u$.

We just proved that $S_{D^{2}, \varepsilon} w_{\varepsilon} \stackrel{E}{\longrightarrow} S_{D^{2}, \Gamma} w$ as $\varepsilon \rightarrow 0$ and this, according to Definition 2.2, implies $S_{D^{2}, \varepsilon} \stackrel{E E}{\longrightarrow} S_{D^{2}, \Gamma}$ as $\varepsilon \rightarrow 0$.

End of the proof of Theorem 4.1. Having already proved Lemma 4.4, it suffices to prove the validity of the compactness property stated in Definition 2.4. Let $w_{\varepsilon} \in V\left(\Omega_{\varepsilon}\right)$ and $\left\|w_{\varepsilon}\right\|_{V\left(\Omega_{\varepsilon}\right)}=1$ for any $\varepsilon>0$. As in Lemma 4.4 we put $u_{\varepsilon}=S_{D^{2}, \varepsilon} w_{\varepsilon}$. By Lemma 4.2 (i) we may assume that, along a sequence converging to zero, there exists $w \in H^{2}(\Omega)$ such that $\left(w_{\varepsilon}\right)_{\mid \Omega} \rightarrow w$ in $H^{2}(\Omega)$. Proceeding as in the proof of Lemma 4.4 we obtain

$$
\left\|u_{\varepsilon}\right\|_{V\left(\Omega_{\varepsilon}\right)} \leq\left(\delta_{1}^{\varepsilon}\right)^{-1}\left\|w_{\varepsilon}\right\|_{V\left(\Omega_{\varepsilon}\right)} \leq C \quad \text { for any } \varepsilon \in\left(0, \varepsilon_{0}\right]
$$

for some constants $\varepsilon_{0}$ and $C$ independent of $\varepsilon$. By Lemma 4.2 (i) we have that $\left\|u_{\varepsilon}\right\|_{H^{2}\left(\Omega_{\varepsilon}\right)} \leq C$ for any $\varepsilon \in\left(0, \varepsilon_{0}\right]$ and hence $\left\{\left(u_{\varepsilon}\right)_{\mid \Omega}\right\}_{0<\varepsilon \leq \varepsilon_{0}}$ is bounded in $H^{2}(\Omega)$; in particular there exists $\widetilde{u} \in H^{2}(\Omega)$ such that $\left(u_{\varepsilon}\right)_{\mid \Omega} \rightarrow \widetilde{u}$ in $H^{2}(\Omega)$ as $\varepsilon \rightarrow 0$ along a sequence. Proceeding as in the proof of Lemma 4.4 one can show that $\widetilde{u} \in H_{0, \Gamma}^{2}(\Omega)$ and it solves (116). In particular, we have that $\widetilde{u}=u:=S_{D^{2}, \Gamma} w$.

We claim that $u_{\varepsilon} \stackrel{E}{\longrightarrow} u$ as $\varepsilon \rightarrow 0$ along a sequence. Proceeding exactly as in the proof of Lemma 4.4 one can verify that (117), (118) and (120) still hold true here. It remains to prove (119) but here we cannot exploit the $E$-convergence of $w_{\varepsilon}$ as we did in the proof of Lemma 4.4. Since $\alpha \geq 1$ we have that $\partial \Omega_{\varepsilon}$ is Lipschitz uniformly with respect to $\varepsilon$ converging to zero. If we look at the proof of [31, Theorem 5.5, Chapter 2] with $k=1$ and $p=2$, we deduce the following uniform estimate

$$
\|v\|_{H^{1 / 2}\left(\partial \Omega_{\varepsilon}\right)} \leq C\|v\|_{H^{1}\left(\Omega_{\varepsilon}\right)} \quad \text { for any } v \in H^{1}\left(\Omega_{\varepsilon}\right)
$$

where $C$ is a positive constant independent of $v$ and $\varepsilon$ small. Introducing a $\varepsilon$-dependent family of extension linear operators from $H^{1}\left(\Omega_{\varepsilon}\right)$ to $H^{1}(W \times(-1,+\infty))$ whose operatorial norms remain bounded as $\varepsilon \rightarrow 0$ (we recall that $\alpha \geq 1$ in the definition of $g_{\varepsilon}$ ), we deduce that if $\left\{v_{\varepsilon}\right\}_{0<\varepsilon \leq \varepsilon_{0}}$ is a family of functions whose $H^{1}\left(\Omega_{\varepsilon}\right)$-norms remain bounded as $\varepsilon \rightarrow 0$ then

$$
\int_{\partial \Omega_{\varepsilon} \backslash\left(\Gamma_{\varepsilon} \cup \partial \Omega\right)} v_{\varepsilon}^{2} d S \rightarrow 0 \quad \text { as } \varepsilon \rightarrow 0
$$

since the surface measure of $\partial \Omega_{\varepsilon} \backslash\left(\Gamma_{\varepsilon} \cup \partial \Omega\right)$ converges to zero as $\varepsilon \rightarrow 0$.

Applying (122) to $\frac{\partial w_{\varepsilon}}{\partial x_{i}}$ and $\frac{\partial u_{\varepsilon}}{\partial x_{i}}$ and recalling that $\left\|u_{\varepsilon}\right\|_{H^{2}\left(\Omega_{\varepsilon}\right)},\left\|w_{\varepsilon}\right\|_{H^{2}\left(\Omega_{\varepsilon}\right)}$ remain bounded as $\varepsilon \rightarrow 0$, we infer that

$$
\int_{\partial \Omega_{\varepsilon} \backslash\left(\Gamma_{\varepsilon} \cup \partial \Omega\right)}\left(w_{\varepsilon}\right)_{\nu}\left(u_{\varepsilon}\right)_{\nu} d S \rightarrow 0 \quad \text { as } \varepsilon \rightarrow 0 .
$$

On the other hand, by (121) applied to $\frac{\partial w_{\varepsilon}}{\partial x_{i}}$ and $\frac{\partial u_{\varepsilon}}{\partial x_{i}}$ combined with the compact embedding $H^{1 / 2}(\partial \Omega \backslash \Gamma) \subset L^{2}(\partial \Omega \backslash \Gamma)$, we have that

$$
\int_{\partial \Omega \backslash \Gamma}\left(w_{\varepsilon}\right)_{\nu}\left(u_{\varepsilon}\right)_{\nu} d S \rightarrow \int_{\partial \Omega \backslash \Gamma} w_{\nu} u_{\nu} d S
$$

along a sequence converging to zero. 
It remains to consider $\int_{\Gamma_{\varepsilon}}\left(w_{\varepsilon}\right)_{\nu}\left(u_{\varepsilon}\right)_{\nu} d S$. By Lemma 4.3, the fact that $\alpha \geq 1$ and $b \in C^{1,1}\left(\mathbb{R}^{N-1}\right)$, we obtain for $\varepsilon$ small enough

$$
\begin{aligned}
& \left|\int_{\Gamma_{\varepsilon}}\left(w_{\varepsilon}\right)_{\nu}\left(u_{\varepsilon}\right) d S\right| \leq\left(\int_{\Gamma_{\varepsilon}}\left(w_{\varepsilon}\right)_{\nu}^{2} d S\right)^{1 / 2}\left(\int_{\Gamma_{\varepsilon}}\left(u_{\varepsilon}\right)_{\nu}^{2} d S\right)^{1 / 2} \\
& \leq\left(\delta_{1}^{\varepsilon}\right)^{-1 / 2}\left\|w_{\varepsilon}\right\|_{V\left(\Omega_{\varepsilon}\right)}\left(\int_{\Gamma_{\varepsilon}}\left(u_{\varepsilon}\right)_{\nu}^{2} d S\right)^{1 / 2} \leq C\left(\sum_{i=1}^{N} \int_{\Gamma_{\varepsilon}}\left|\frac{\partial u_{\varepsilon}}{\partial x_{i}}\right|^{2} d S\right)^{1 / 2} \\
& =C\left(\sum_{i=1}^{N} \int_{W}\left|\frac{\partial u_{\varepsilon}}{\partial x_{i}}\left(x^{\prime}, g_{\varepsilon}\left(x^{\prime}\right)\right)\right|^{2} \sqrt{1+\left|\nabla_{x^{\prime}} g_{\varepsilon}\left(x^{\prime}\right)\right|^{2}} d x^{\prime}\right)^{1 / 2} \leq C\left(\sum_{i=1}^{N} \int_{W}\left|\frac{\partial u_{\varepsilon}}{\partial x_{i}}\left(x^{\prime}, g_{\varepsilon}\left(x^{\prime}\right)\right)\right|^{2} d x^{\prime}\right)^{1 / 2}
\end{aligned}
$$

where $C$ denotes a constant independent of $\varepsilon$ which may vary in every estimate.

We claim that $\int_{W}\left|\frac{\partial u_{\varepsilon}}{\partial x_{i}}\left(x^{\prime}, g_{\varepsilon}\left(x^{\prime}\right)\right)\right|^{2} d x^{\prime} \rightarrow 0$ as $\varepsilon \rightarrow 0$. Indeed, we have

$$
\int_{W}\left|\frac{\partial u_{\varepsilon}}{\partial x_{i}}\left(x^{\prime}, g_{\varepsilon}\left(x^{\prime}\right)\right)\right|^{2} d x^{\prime} \leq 2 \int_{W}\left|\frac{\partial u_{\varepsilon}}{\partial x_{i}}\left(x^{\prime}, g_{\varepsilon}\left(x^{\prime}\right)\right)-\frac{\partial u_{\varepsilon}}{\partial x_{i}}\left(x^{\prime}, 0\right)\right|^{2} d x^{\prime}+2 \int_{W}\left|\frac{\partial u_{\varepsilon}}{\partial x_{i}}\left(x^{\prime}, 0\right)\right|^{2} d x^{\prime} \text {. }
$$

The second term at the right hand side of (126) converges to zero as one can verify combining the fact that $\left(u_{\varepsilon}\right)_{\mid \Omega} \rightarrow u$ in $H^{2}(\Omega)$ and $u \in H_{0, \Gamma}^{2}(\Omega)$, with compactness of the trace map. With the first term at the right hand side of (126) we proceed in this way

$$
\begin{aligned}
& \int_{W}\left|\frac{\partial u_{\varepsilon}}{\partial x_{i}}\left(x^{\prime}, g_{\varepsilon}\left(x^{\prime}\right)\right)-\frac{\partial u_{\varepsilon}}{\partial x_{i}}\left(x^{\prime}, 0\right)\right|^{2} d x^{\prime}=\int_{W}\left|\int_{0}^{g_{\varepsilon}\left(x^{\prime}\right)} \frac{\partial^{2} u_{\varepsilon}}{\partial x_{N} \partial x_{i}}\left(x^{\prime}, x_{N}\right) d x_{N}\right|^{2} d x^{\prime} \\
& \leq\left.\left.\int_{W}\left|g_{\varepsilon}\left(x^{\prime}\right)\right|\left|\int_{0}^{g_{\varepsilon}\left(x^{\prime}\right)}\right| \frac{\partial^{2} u_{\varepsilon}}{\partial x_{N} \partial x_{i}}\left(x^{\prime}, x_{N}\right)\right|^{2} d x_{N}\left|d x^{\prime} \leq \varepsilon^{\alpha}\|b\|_{L^{\infty}\left(\mathbb{R}^{N-1}\right)} \int_{\Omega_{\varepsilon} \backslash \Omega}\right| \frac{\partial^{2} u_{\varepsilon}}{\partial x_{N} \partial x_{i}}\right|^{2} d x \\
& \leq \varepsilon^{\alpha}\|b\|_{L^{\infty}\left(\mathbb{R}^{N-1}\right)}\left\|u_{\varepsilon}\right\|_{H^{2}\left(\Omega_{\varepsilon}\right)}^{2} \rightarrow 0 \quad \text { as } \varepsilon \rightarrow 0 .
\end{aligned}
$$

The proof of the claim follows by combining (126) and (127). In turn, the claim combined with (125) implies

$$
\int_{\Gamma_{\varepsilon}}\left(w_{\varepsilon}\right)_{\nu}\left(u_{\varepsilon}\right)_{\nu} d S \rightarrow 0 \quad \text { as } \varepsilon \rightarrow 0
$$

Now, combining (123), (124) and (128), we obtain

$$
\int_{\partial \Omega_{\varepsilon}}\left(w_{\varepsilon}\right)_{\nu}\left(u_{\varepsilon}\right)_{\nu} d S \rightarrow \int_{\partial \Omega \backslash \Gamma} w_{\nu} u_{\nu} d S
$$

as $\varepsilon \rightarrow 0$ along a sequence. Since $u_{\varepsilon}$ satisfies (118) and $u \in H_{0, \Gamma}^{2}(\Omega)$ is a weak solution of (116), from (129) we obtain

$$
\int_{\Omega_{\varepsilon}}\left|D^{2} u_{\varepsilon}\right|^{2} d x=\int_{\partial \Omega_{\varepsilon}}\left(w_{\varepsilon}\right)_{\nu}\left(u_{\varepsilon}\right)_{\nu} d S \rightarrow \int_{\partial \Omega \backslash \Gamma} w_{\nu} u_{\nu} d S=\int_{\Omega}\left|D^{2} u\right|^{2} d x
$$

as $\varepsilon \rightarrow 0$ along a sequence.

Since (117) still holds true for $u_{\varepsilon}$, by (130), it follows that $u_{\varepsilon} \stackrel{E}{\rightarrow} u$ as $\varepsilon \rightarrow 0$ along a sequence. We have proved that $\left\{S_{D^{2}, \varepsilon} w_{\varepsilon}\right\}$ is precompact in the sense of Definition 2.3. The proof of the theorem is now a consequence of Lemma 4.4 and Definition 2.4 . 
4.2. Case $\alpha=3 / 2$ : strange term. Assume that $\Omega, \Omega_{\varepsilon}, \Gamma$ and $\Gamma_{\varepsilon}$ are as above. We set $\Sigma_{\varepsilon}=\partial \Omega_{\varepsilon} \backslash \Gamma_{\varepsilon}$ and $\Sigma=\partial \Omega \backslash \Gamma$. In this subsection we discuss the following modified Steklov problem:

$$
\begin{cases}\Delta^{2} u=0, & \text { in } \Omega_{\varepsilon}, \\ u=0, & \text { on } \partial \Omega_{\varepsilon}, \\ u_{\nu}=0, & \text { on } \Sigma_{\varepsilon}, \\ \Delta u-K(x) u_{\nu}=\delta u_{\nu}, & \text { on } \Gamma_{\varepsilon} .\end{cases}
$$

The third condition $u_{\nu}=0$ on $\Sigma_{\varepsilon}$ is used here to simplify our arguments as well as to avoid a few annoying technicalities which seem not particularly interesting for the specific case under discussion.

The eigenvalues of problem (131) are the reciprocals of the eigenvalues of a Navier-to-Neumann map $N_{\Omega_{\varepsilon}}$ that we now define. Namely, given $f \in L^{2}\left(\Gamma_{\varepsilon}\right)$ consider the solution $u_{f}=u$ to the following modified Navier problem

$$
\begin{cases}\Delta^{2} u=0, & \text { in } \Omega_{\varepsilon}, \\ u=0, & \text { on } \partial \Omega_{\varepsilon}, \\ u_{\nu}=0, & \text { on } \Sigma_{\varepsilon}, \\ \Delta u-K(x) u_{\nu}=f, & \text { on } \Gamma_{\varepsilon},\end{cases}
$$

which means that $u \in H_{0, \Sigma_{\varepsilon}}^{2}\left(\Omega_{\varepsilon}\right) \cap H_{0}^{1}\left(\Omega_{\varepsilon}\right)$ is the solution to the weak problem

$$
\int_{\Omega_{\varepsilon}} D^{2} u: D^{2} \varphi d x=\int_{\Gamma_{\varepsilon}} f \frac{\partial \varphi}{\partial \nu} d S, \quad \forall \varphi \in H_{0, \Sigma_{\varepsilon}}^{2}\left(\Omega_{\varepsilon}\right) \cap H_{0}^{1}\left(\Omega_{\varepsilon}\right) .
$$

Here $H_{0, \Sigma_{\varepsilon}}^{2}\left(\Omega_{\varepsilon}\right)$ is defined as in (112) with $\Omega$ replaced by $\Omega_{\varepsilon}$ and $\Gamma$ replaced by $\Sigma_{\varepsilon}$. Then we consider the function $N_{\Omega_{\varepsilon}}$ from $L^{2}\left(\Gamma_{\varepsilon}\right)$ to itself defined by $N_{\Omega_{\varepsilon}} f=\frac{\partial u_{f}}{\partial \nu}$ for all $f \in L^{2}\left(\Gamma_{\varepsilon}\right)$ where $u_{f}$ is the solution to (133). Here we analyse the behaviour of the map $N_{\Omega_{\varepsilon}}$ as $\varepsilon \rightarrow 0$ in the case $\alpha=3 / 2$.

The limiting problem involves a strange factor $\gamma$, which is called 'strange curvature' in [4]. We recall that $\gamma$ is defined by

$$
\gamma=\int_{Y \times(-\infty, 0)}\left|D^{2} V\right|^{2} d y
$$

and the function $V$ is $Y$-periodic in the variables $y^{\prime}$ and satisfies the following microscopic problem

$$
\begin{cases}\Delta^{2} V=0, & \text { in } Y \times(-\infty, 0), \\ V\left(y^{\prime}, 0\right)=b\left(y^{\prime}\right), & \text { on } Y, \\ \frac{\partial^{2} V}{\partial y_{N}^{2}}\left(y^{\prime}, 0\right)=0, & \text { on } Y .\end{cases}
$$

It turns out that the limiting functional for the maps $N_{\Omega_{\varepsilon}}$ as $\varepsilon \rightarrow 0$ is the map $S_{\Omega}$ defined as follows. Given $f \in L^{2}(\Gamma)$, consider the solution $\tilde{u}_{f}=u$ to the problem

$$
\begin{cases}\Delta^{2} u=0, & \text { in } \Omega, \\ u=0, & \text { on } \partial \Omega, \\ u_{\nu}=0, & \text { on } \Sigma, \\ \Delta u-K(x) u_{\nu}+\gamma u_{\nu}=f, & \text { on } \Gamma .\end{cases}
$$

Then $S_{\Omega}$ is the map from $L^{2}(\Gamma)$ to itself defined by $S_{\Omega} f=\frac{\partial \tilde{u}_{f}}{\partial \nu}$ for all $f \in L^{2}(\Gamma)$. Since the trace operator is compact we have that both operators $N_{\Omega_{\varepsilon}}$ and $S_{\Omega}$ are compact.

We now consider a family of operators $E_{\varepsilon}$ from $L^{2}(\Gamma)$ to $L^{2}\left(\Gamma_{\varepsilon}\right)$ defined by $E_{\varepsilon}(f)\left(x^{\prime}, g_{\varepsilon}\left(x^{\prime}\right)\right)=f\left(x^{\prime}, 0\right)$ for all $x^{\prime} \in W$ and all $f \in L^{2}(\Gamma)$. It is obvious that if $\alpha=3 / 2$ then $g_{\varepsilon}$ converges to zero uniformly together with its first derivatives hence

$$
\left\|E_{\varepsilon} f\right\|_{L^{2}\left(\Gamma_{\varepsilon}\right)} \rightarrow\|f\|_{L^{2}(\Gamma)}
$$


as $\varepsilon \rightarrow 0$, which means that condition (11) is satisfied. With reference to these operators $E_{\varepsilon}$, the following theorem holds.

Theorem 4.5. If $\alpha=3 / 2$ then $N_{\Omega_{\varepsilon}} \stackrel{C}{\longrightarrow} S_{\Omega}$ as $\varepsilon \rightarrow 0$, hence the spectrum of problem (131) converges to the spectrum of (136) in the sense of Theorem 2.5

Proof. Let $f_{\varepsilon} \in L^{2}\left(\Gamma_{\varepsilon}\right)$ be $E$-convergent to $f \in L^{2}(\Gamma)$ as $\varepsilon \rightarrow 0$ in the sense of Definition 2.1 Let $u_{\varepsilon}$ be the solution to problem (133) with $f$ replaced by $f_{\varepsilon}$. Using $u_{\varepsilon}$ as a test function in (133), we get that $\left\|u_{\varepsilon}\right\|_{H^{2}\left(\Omega_{\varepsilon}\right)}$ is uniformly bounded, hence there exists $u \in H_{0, \Sigma}^{2}(\Omega) \cap H_{0}^{1}(\Omega)$ such that possibly passing to a subsequence, $u_{\varepsilon} \rightarrow u$ weakly in $H^{2}(\Omega)$ and strongly in $H^{1}(\Omega)$. We now prove that $u$ satisfies problem (136).

To do so, following [4, § 8.1] we define a diffeomorphism $\Phi_{\varepsilon}$ from $\bar{\Omega}_{\varepsilon}$ onto $\bar{\Omega}$. Namely, $\Phi_{\varepsilon}\left(x^{\prime}, x_{N}\right)=$ $\left(x^{\prime}, x_{N}-h_{\varepsilon}\left(x^{\prime}, x_{N}\right)\right)$ for all $\left(x^{\prime}, x_{N}\right) \in \bar{\Omega}_{\varepsilon}$ where

$$
h_{\varepsilon}\left(x^{\prime}, x_{N}\right)= \begin{cases}0, & \text { if }-1 \leq x_{N} \leq-\varepsilon \\ g_{\varepsilon}\left(x^{\prime}\right)\left(\frac{x_{N}+\varepsilon}{g_{\varepsilon}\left(x^{\prime}\right)+\varepsilon}\right)^{3}, & \text { if }-\varepsilon<x_{N} \leq g_{\varepsilon}\left(x^{\prime}\right) .\end{cases}
$$

It is convenient to denote by $K_{\varepsilon}$ the set where $\Phi_{\varepsilon}$ coincides with the identity, that is $K_{\varepsilon}=\left\{\left(x^{\prime}, x_{N}\right) \in\right.$ $\left.W \times \mathbb{R}:-1<x_{N} \leq-\varepsilon\right\}$.

Let $\varphi \in H_{0, \Sigma}^{2}(\Omega) \cap H_{0}^{1}(\Omega)$ be fixed. We set $\varphi_{\varepsilon}=\varphi \circ \Phi_{\varepsilon}$ and we note that $\varphi_{\varepsilon} \in H_{0, \Sigma_{\varepsilon}}^{2}\left(\Omega_{\varepsilon}\right) \cap H_{0}^{1}\left(\Omega_{\varepsilon}\right)$. Using $\varphi_{\varepsilon}$ as a test function in (133) we get

$$
\int_{K_{\varepsilon}} D^{2} u_{\varepsilon}: D^{2} \varphi d x+\int_{\Omega_{\varepsilon} \backslash K_{\varepsilon}} D^{2} u_{\varepsilon}: D^{2} \varphi_{\varepsilon} d x=\int_{\Omega_{\varepsilon}} D^{2} u_{\varepsilon}: D^{2} \varphi_{\varepsilon} d x=\int_{\Gamma_{\varepsilon}} f_{\varepsilon} \frac{\partial \varphi_{\varepsilon}}{\partial \nu} d S .
$$

It is proved in [4, Theorem 8.72] that

$$
\lim _{\varepsilon \rightarrow 0} \int_{\Omega_{\varepsilon} \backslash K_{\varepsilon}} D^{2} u_{\varepsilon}: D^{2} \varphi_{\varepsilon} d x=\gamma \int_{W} \frac{\partial u}{\partial x_{N}}\left(x^{\prime}, 0\right) \frac{\partial \varphi}{\partial x_{N}}\left(x^{\prime}, 0\right) d x^{\prime},
$$

where $\gamma$ is as in the statement. Note that a careful inspection of the arguments in [4, § 8] shows that the definition of $\gamma$ is not affected by our Dirichlet boundary conditions on $\Sigma_{\varepsilon}$. Thus

$$
\lim _{\varepsilon \rightarrow 0} \int_{\Omega_{\varepsilon}} D^{2} u_{\varepsilon}: D^{2} \varphi_{\varepsilon} d x=\int_{\Omega} D^{2} u: D^{2} \varphi d x+\gamma \int_{W} \frac{\partial u}{\partial x_{N}}\left(x^{\prime}, 0\right) \frac{\partial \varphi}{\partial x_{N}}\left(x^{\prime}, 0\right) d x^{\prime} .
$$

We now consider the integral in the right-hand side of (139). We have

$$
\begin{aligned}
& \left|\int_{\Gamma_{\varepsilon}} f_{\varepsilon} \frac{\partial \varphi_{\varepsilon}}{\partial \nu} d S-\int_{\Gamma} f \frac{\partial \varphi}{\partial \nu} d S\right| \leq \int_{\Gamma_{\varepsilon}}\left|f_{\varepsilon}-E_{\varepsilon} f\right|\left|\frac{\partial \varphi_{\varepsilon}}{\partial \nu}\right| d S+\left|\int_{\Gamma_{\varepsilon}} E_{\varepsilon} f \frac{\partial \varphi_{\varepsilon}}{\partial \nu} d S-\int_{\Gamma} f \frac{\partial \varphi}{\partial \nu} d S\right| \\
& \leq C\left\|f_{\varepsilon}-E_{\varepsilon} f\right\|_{L^{2}\left(\Gamma_{\varepsilon}\right)}+\left|\int_{\Gamma_{\varepsilon}} E_{\varepsilon} f \frac{\partial \varphi_{\varepsilon}}{\partial \nu} d S-\int_{\Gamma} f \frac{\partial \varphi}{\partial \nu} d S\right| \\
& =o(1)+\left|\int_{W} f\left(x^{\prime}, 0\right) \frac{\partial \varphi_{\varepsilon}}{\partial \nu}\left(x^{\prime}, g_{\varepsilon}\left(x^{\prime}\right)\right) \sqrt{1+\left|\nabla_{x^{\prime}} g_{\varepsilon}\left(x^{\prime}\right)\right|^{2}} d x^{\prime}-\int_{W} f\left(x^{\prime}, 0\right) \frac{\partial \varphi}{\partial \nu}\left(x^{\prime}, 0\right) d x^{\prime}\right|=o(1),
\end{aligned}
$$

where $C>0$ is independent of $\varepsilon$. It follows by (141)-(142) that function $u$ satisfies the problem

$$
\int_{\Omega} D^{2} u: D^{2} \varphi d x+\gamma \int_{W} \frac{\partial u}{\partial x_{N}}\left(x^{\prime}, 0\right) \frac{\partial \varphi}{\partial x_{N}}\left(x^{\prime}, 0\right) d x^{\prime}=\int_{\Gamma} f \frac{\partial \varphi}{\partial \nu} d S .
$$

We have now to prove that $\frac{\partial u_{\varepsilon}}{\partial \nu_{\varepsilon}}$ is $E$-convergent to $\frac{\partial u}{\partial \nu}$. We note that 


$$
\begin{aligned}
\int_{\partial \Omega_{\varepsilon}}\left|\frac{\partial u_{\varepsilon}}{\partial \nu}-E_{\varepsilon} \frac{\partial u}{\partial \nu}\right|^{2} d S=\int_{W} \mid \frac{\partial u_{\varepsilon}}{\partial \nu}\left(x^{\prime}, g_{\varepsilon}\left(x^{\prime}\right)\right) & -\left.\frac{\partial u}{\partial \nu}\left(x^{\prime}, 0\right)\right|^{2} \sqrt{1+\left|\nabla g_{\varepsilon}\left(x^{\prime}\right)\right|^{2}} d x^{\prime} \\
& =\int_{W}\left|\frac{\partial u_{\varepsilon}}{\partial \nu}\left(x^{\prime}, g_{\varepsilon}\left(x^{\prime}\right)\right)-\frac{\partial u}{\partial \nu}\left(x^{\prime}, 0\right)\right|^{2} d x^{\prime}+o(1) .
\end{aligned}
$$

By the Fundamental Theorem of Calculus we have that

$$
\left\|\nabla u_{\varepsilon}\left(x^{\prime}, g_{\varepsilon}\left(x^{\prime}\right)\right)-\nabla u_{\varepsilon}\left(x^{\prime}, 0\right)\right\|_{L^{2}(W)} \leq c \varepsilon^{\alpha / 2}\left\|u_{\varepsilon}\right\|_{H^{2}\left(\Omega_{\varepsilon} \backslash \Omega\right)}=O\left(\varepsilon^{\alpha / 2}\right)
$$

as $\varepsilon \rightarrow 0$. On the other hand, by the compactness of the trace operator we have that possibly passing to a subsequence

$$
\left\|\nabla u_{\varepsilon}\left(x^{\prime}, 0\right)-\nabla u\left(x^{\prime}, 0\right)\right\|_{L^{2}(W)} \rightarrow 0
$$

$\varepsilon \rightarrow 0$. By combining (144)-(146) we conclude that $\int_{\partial \Omega_{\varepsilon}}\left|\frac{\partial u_{\varepsilon}}{\partial \nu}-E \frac{\partial u}{\partial \nu}\right|^{2} d S \rightarrow 0$ as $\varepsilon \rightarrow 0$ hence $\frac{\partial u_{\varepsilon}}{\partial \nu}$ is $E$-convergent to $\frac{\partial u}{\partial \nu}$.

In order to conclude the proof, it suffices to observe that if $f_{\varepsilon}$ is a sequence with $\left\|f_{\varepsilon}\right\|_{L^{2}\left(\Gamma_{\varepsilon}\right)}=1$ for all $\varepsilon>0$, then the corresponding solutions $u_{\varepsilon}$ have uniformly bounded $H^{2}$ norms, hence exploiting the compactness of the trace operator as above one can easily prove that $\frac{\partial u_{\varepsilon}}{\partial \nu}$ has an $E$-convergent subsequence.

4.3. Another way of viewing the trichotomy. In this subsection we consider the eigenvalue problem (131) and we re-interpret the results of this section adapting the analysis of Subsection 4.1 to this specific case. This allows to state a trichotomy result for the eigenvalues which we believe is quite transparent. Namely, we have the following theorem.

Theorem 4.6. Let $\Omega_{\varepsilon}, \varepsilon \geq 0$ be as above, with $\Omega_{0}=\Omega$. Let $\lambda_{n}(\varepsilon), n \in \mathbb{N}$, be the eigenvalues of problems (131) for $\varepsilon \geq 0$. Then the following statements hold:

(i) If $\alpha>3 / 2$ then $\lambda_{n}(\varepsilon) \rightarrow \lambda_{n}(0)$, as $\varepsilon \rightarrow 0$.

(ii) If $\alpha=3 / 2$ then $\lambda_{n}(\varepsilon) \rightarrow \lambda_{n}(0)+\gamma$ as $\varepsilon \rightarrow 0$, where $\gamma$ is as in (134).

(iii) If $1 \leq \alpha<3 / 2$ then $\lambda_{n}(\varepsilon) \rightarrow \infty$, as $\varepsilon \rightarrow 0$.

Proof. The proof of (i) is exactly the same proof of Corollary 4.1 with obvious minor modifications. Statement (ii) is a straightforward application of Theorem 4.5. We now prove statement (iii). We proceed as in Subsection 4.1 replacing the energy spaces $H^{2}\left(\Omega_{\varepsilon}\right) \cap H_{0}^{1}\left(\Omega_{\varepsilon}\right)$ and $H_{0, \Gamma}^{2}(\Omega)$ by $H_{0, \Sigma_{\varepsilon}}^{2}\left(\Omega_{\varepsilon}\right) \cap H_{0}^{1}\left(\Omega_{\varepsilon}\right)$ and $H_{0}^{2}(\Omega)$ respectively. Accordingly, the operators $S_{D^{2}, \varepsilon}$ and $S_{D^{2}, \Gamma}$ used in Subsection 4 .1 have to be re-defined in an obvious way (formally, it is the same way) taking into account the new energy spaces. Let's call $\tilde{S}_{D^{2}, \varepsilon}$ and $\tilde{S}_{D^{2}, \Gamma}$ the new operators replacing $S_{D^{2}, \varepsilon}$ and $S_{D^{2}, \Gamma}$, respectively. Having a closer look at the definition of $\tilde{S}_{D^{2}, \Gamma}$, one realises that actually $\tilde{S}_{D^{2}, \Gamma}$ is the operator identically equal to zero: indeed, the map $J_{\Gamma}$ used in the definition of $S_{D^{2}, \Gamma}$ vanishes on $H_{0}^{2}(\Omega)$. Thus, by the same argument used in the proof of Theorem 4.1 we have that $\tilde{S}_{D^{2}, \varepsilon} \stackrel{C}{\longrightarrow} 0$ as $\varepsilon \rightarrow 0$. Assume now by contradiction that there exists a sequence $\varepsilon_{k} \rightarrow 0$ such that for some $n \in \mathbb{N}$ we have $\sup _{k \in \mathbb{N}} \lambda_{n}\left(\varepsilon_{k}\right)<\infty$. Using the the Poincaré inequality, one can see that $\inf _{k \in \mathbb{N}} \lambda_{n}\left(\varepsilon_{k}\right)>0$, see also Lemma4.3. Now, we set $\mu_{n}\left(\varepsilon_{k}\right)=1 / \lambda_{n}\left(\varepsilon_{k}\right)$ and we observe that the sequence $\mu_{n}\left(\varepsilon_{k}\right), k \in \mathbb{N}$ is bounded away from zero and infinity. Thus, possibly passing to a subsequence, there exists $\mu>0$ such that $\mu_{n}\left(\varepsilon_{k}\right) \rightarrow \mu$ as $k \rightarrow \infty$. Since $\mu_{n}\left(\varepsilon_{k}\right)$ is an eigenvalue of $\tilde{S}_{D^{2}, \varepsilon_{k}}$ and $\tilde{S}_{D^{2}, \varepsilon_{k}} \stackrel{C}{\longrightarrow} 0$, by Vainikko [38, Theorem 6.1] it follows that $\mu$ is an eigenvalue of the zero operator, a contradiction.

\section{NAVIER-TYPE PROBLEMS AND NAVIER-TO-NEUMANN MAPS}

The main aim of this section is to study the stability of the inverse of a Dirichlet-to-Neumann-type map associated with problem (2) under weak assumptions on the convergence of the varying domains. As we 
have mentioned in the introduction, this map is called here Navier-to-Neumann map. To do so, we begin with discussing the stability of the Navier problem under domain perturbations of general type.

5.1. Navier problems. Given a sufficiently smooth bounded domain $\Omega$ in $\mathbb{R}^{N}$, the classical Navier problem for the biharmonic operator reads

$$
\begin{cases}\Delta^{2} u=0, & \text { in } \Omega, \\ u=0, & \text { on } \partial \Omega, \\ \Delta u=f, & \text { on } \partial \Omega .\end{cases}
$$

In a classical setting, it would be natural to require at least that $\Omega$ is of class $C^{0,1}$ and that $f \in H^{1 / 2}(\partial \Omega)$. Actually, since the trace operator is surjective, one could directly assume that $f \in H^{1}(\Omega)$.

As it is well-known, problem (147) could be recast in the form of a system by setting $v:=\Delta u$ and solving

$$
\left\{\begin{array} { l l } 
{ \Delta v = 0 , } & { \text { in } \Omega } \\
{ v = f , } & { \text { on } \partial \Omega }
\end{array} \text { and } \left\{\begin{array}{ll}
\Delta u=v, & \text { in } \Omega \\
u=0, & \text { on } \partial \Omega .
\end{array}\right.\right.
$$

Accordingly, in order to prove that problem (147) is stable under domain perturbations, one may think of exploiting the properties of both Poisson and Dirichlet problems in (148) which are stable under sufficiently regular boundary perturbations. However, since we aim at considering a rather general class of domain perturbations as well as at comparing problem (147) with problem (184), we prefer to adopt another point of view which has its own interest, and which is based on the energy quadratic form naturally associated with (147). This point of view allows to relax the boundary regularity assumptions on $\Omega$ even more.

Namely, we interpret problem (147) in a weak sense by formulating it as follows

$$
\int_{\Omega} \Delta u \Delta \varphi d x=\int_{\Omega}(f \Delta \varphi+\nabla f \nabla \varphi) d x, \text { for all } \varphi \in H(\Delta, \Omega) \cap H_{0}^{1}(\Omega),
$$

where the unknown $u$ has to be considered in $H(\Delta, \Omega) \cap H_{0}^{1}(\Omega)$. Note that our weak formulation is motivated by the fact that if $\Omega$ is regular enough, for example as in Lemma 2.7 (iii), then $H(\Delta, \Omega) \cap H_{0}^{1}(\Omega)=$ $H^{2}(\Omega) \cap H_{0}^{1}(\Omega)$ hence equality (149) could be re-written as

$$
\int_{\Omega} \Delta u \Delta \varphi d x=\int_{\partial \Omega} f \varphi_{\nu} d S
$$

which is a more familiar way of writing the variational formulation for (147). In fact the right-hand side of (149) is the standard way of defining the normal derivative for a function in $H(\Delta, \Omega)$ as an element of $H^{-1 / 2}(\partial \Omega)$, see (173) below, see also e.g., [26].

The following lemma shows that problem 149 is well-posed even without any boundary regularity assumption on $\Omega$.

Lemma 5.1. Let $\Omega$ be a domain in $\mathbb{R}^{N}$ such that the Poincaré inequality holds and let $f \in H^{1}(\Omega)$. Then problem (149) is well-posed in the sense of Hadamard, that is there exists a unique solution u in $H(\Delta, \Omega) \cap$ $H_{0}^{1}(\Omega)$ which depends continuously on $f$.

Proof. We simply observe that by Lemma2.7 the map $\varphi \mapsto \int_{\Omega}(f \Delta \varphi+\nabla f \nabla \varphi) d x$ is an element of the dual of $H(\Delta, \Omega) \cap H_{0}^{1}(\Omega)$ which depends continuously on $f \in H^{1}(\Omega)$. Then the proof easily follows by the Riesz Theorem applied to the Hilbert space $H(\Delta, \Omega) \cap H_{0}^{1}(\Omega)$.

We now consider a family of bounded domains $\Omega_{\varepsilon}, \varepsilon>0$ and the corresponding boundary value problems

$$
\begin{cases}\Delta^{2} u_{\varepsilon}=0, & \text { in } \Omega_{\varepsilon}, \\ u_{\varepsilon}=0, & \text { on } \partial \Omega_{\varepsilon}, \\ \Delta u_{\varepsilon}=f_{\varepsilon}, & \text { on } \partial \Omega_{\varepsilon},\end{cases}
$$


in the unknown $u_{\varepsilon} \in H\left(\Delta, \Omega_{\varepsilon}\right) \cap H_{0}^{1}\left(\Omega_{\varepsilon}\right)$, where $f_{\varepsilon} \in H^{1}\left(\Omega_{\varepsilon}\right)$, with the understanding that problem (151) has to be interpreted in the weak sense as above.

We shall assume that the domains $\Omega_{\varepsilon}$ compactly converge to $\Omega$ and that $\Omega$ is regular (stable) in the sense of the following definition which goes back to Keldysh (cf. [12, § 4.9]).

Definition 5.2. We say that the domains $\Omega_{\varepsilon}, \varepsilon>0$, compactly converge to a domain $\Omega$ as $\varepsilon \rightarrow 0$ if for any compact set $K \subset \Omega \cup\left(\mathbb{R}^{N} \backslash \bar{\Omega}\right)$ there exists $\varepsilon_{K}>0$ such that $K \subset \Omega_{\varepsilon} \cup\left(\mathbb{R}^{N} \backslash \bar{\Omega}_{\varepsilon}\right)$ for all $\left.\varepsilon \in\right] 0, \varepsilon_{K}[$. Moreover, we say that $\Omega$ is stable if any function in $H^{1}\left(\mathbb{R}^{N}\right)$ vanishing almost everywhere on $\mathbb{R}^{N} \backslash \bar{\Omega}$ belongs to $H_{0}^{1}(\Omega)$.

It is well-known that if $\Omega$ is of class $C^{0,1}$ then $\Omega$ is stable. We refer to [12] for more information. Then we have the following stability result. Here we denote by $v_{0}$ the extension-by-zero of a function $v$ outside its natural domain of definition.

Theorem 5.3. Let $D$ be a fixed bounded set and let $\Omega_{\varepsilon}, \varepsilon>0$, and $\Omega$ be domains contained in $D$. Assume that the sets $\Omega_{\varepsilon}$ compactly converge to $\Omega$ as $\varepsilon \rightarrow 0$ and that $\Omega$ is stable. If the data $f_{\varepsilon}$ converge to $f$ in the sense that

$$
\left\|f_{\varepsilon}-f_{0}\right\|_{L^{2}\left(\Omega_{\varepsilon}\right)}, \quad\left\|\nabla f_{\varepsilon}-(\nabla f)_{0}\right\|_{L^{2}\left(\Omega_{\varepsilon}\right)} \rightarrow 0, \text { as } \varepsilon \rightarrow 0
$$

then the solutions $u_{\varepsilon}$ of problems (151) converge to the solution $u$ of problem (147) in the sense that

$$
\left\|u_{\varepsilon}-u_{0}\right\|_{L^{2}\left(\Omega_{\varepsilon}\right)},\left\|\nabla u_{\varepsilon}-(\nabla u)_{0}\right\|_{L^{2}\left(\Omega_{\varepsilon}\right)},\left\|\Delta u_{\varepsilon}-(\Delta u)_{0}\right\|_{L^{2}\left(\Omega_{\varepsilon}\right)} \rightarrow 0, \text { as } \varepsilon \rightarrow 0 .
$$

Proof. By assumption, it follows that

$$
\left\|f_{\varepsilon}\right\|_{L^{2}\left(\Omega_{\varepsilon}\right)},\left\|\nabla f_{\varepsilon}\right\|_{L^{2}\left(\Omega_{\varepsilon}\right)} \leq C,
$$

for all $\varepsilon>0$ sufficiently small. Using $u_{\varepsilon}$ as a test function in (149) we have

$$
\int_{\Omega_{\varepsilon}}\left|\Delta u_{\varepsilon}\right|^{2} d x=\int_{\Omega_{\varepsilon}}\left(f_{\varepsilon} \Delta u_{\varepsilon}+\nabla f_{\varepsilon} \nabla u_{\varepsilon}\right) d x
$$

which, combined with (14) and (154), allows to conclude that

$$
\left\|u_{\varepsilon}\right\|_{L^{2}\left(\Omega_{\varepsilon}\right)},\left\|\nabla u_{\varepsilon}\right\|_{L^{2}\left(\Omega_{\varepsilon}\right)},\left\|\Delta u_{\varepsilon}\right\|_{L^{2}\left(\Omega_{\varepsilon}\right)} \leq C,
$$

for all $\varepsilon>0$ sufficiently small. Since $D$ is bounded, by the Rellich-Kondrakov Theorem, $H_{0}^{1}(D)$ is compactly embedded in $L^{2}(D)$. This combined with the fact that $\left(u_{\varepsilon}\right)_{0} \in H_{0}^{1}(D)$ allows to conclude that there exists $u \in H_{0}^{1}(D)$ such that possibly passing to a subsequence

$$
\left(u_{\varepsilon}\right)_{0} \rightarrow u, \text { in } L^{2}(D), \text { and }\left(u_{\varepsilon}\right)_{0} \rightarrow u, \text { in } H^{1}(D)
$$

as $\varepsilon \rightarrow 0$. By the compact convergence of the domains $\Omega_{\varepsilon}$ to the set $\Omega$ it follows that $u=0$ almost everywhere on $D \backslash \bar{\Omega}$. Since $\Omega$ is stable, this implies that $u \in H_{0}^{1}(\Omega)$ and that $u=0$ almost everywhere on $D \backslash \Omega$, see [12, p. 117]. By (156) it follows that possibly passing to a subsequence there exists $v \in L^{2}(D)$ such that $\left(\Delta u_{\varepsilon}\right)_{0} \rightarrow v$ in $L^{2}(D)$. Let $\psi \in C_{c}^{\infty}(\Omega)$ be fixed. By the compact convergence of the domains $\Omega_{\varepsilon}$ to the set $\Omega$ it follows that for all $\varepsilon>0$ sufficiently small, $\psi \in C_{c}^{\infty}\left(\Omega_{\varepsilon}\right)$ hence $\int_{\Omega_{\varepsilon}} u_{\varepsilon} \Delta \psi d x=\int_{\Omega_{\varepsilon}} \Delta u_{\varepsilon} \psi d x$ and $\int_{\Omega} u_{\varepsilon} \Delta \psi d x=\int_{\Omega} \Delta u_{\varepsilon} \psi d x$. Passing to the limit in the last equality as $\varepsilon \rightarrow 0$, it follows that $\int_{\Omega} u \Delta \psi d x=$ $\int_{\Omega} v \psi d x$ which, by the arbitrary choice of $\psi$, implies that $u \in H(\Delta, \Omega) \cap H_{0}^{1}(\Omega)$ and $\Delta u=v$. In particular

$$
\left(\left(\Delta u_{\varepsilon}\right)_{0}\right)_{\left.\right|_{\Omega}} \rightarrow \Delta u, \text { in } L^{2}(\Omega),
$$

as $\varepsilon \rightarrow 0$.

Let $\varphi \in H(\Delta, \Omega) \cap H_{0}^{1}(\Omega)$ be fixed. For every $\varepsilon>0$ we consider the function $\varphi_{\varepsilon} \in H\left(\Delta, \Omega_{\varepsilon}\right) \cap H_{0}^{1}\left(\Omega_{\varepsilon}\right)$ uniquely defined as the solution to the problem

$$
\begin{cases}\Delta \varphi_{\varepsilon}=(\Delta \varphi)_{0}, & \text { in } \Omega_{\varepsilon}, \\ \varphi_{\varepsilon}=0, & \text { on } \partial \Omega_{\varepsilon} .\end{cases}
$$


This means that $\varphi_{\varepsilon}$ satisfies the weak equation

$$
\int_{\Omega_{\varepsilon}} \nabla \varphi_{\varepsilon} \nabla \eta d x=\int_{\Omega_{\varepsilon}}(\Delta \varphi)_{0} \eta d x, \text { for all } \eta \in H_{0}^{1}\left(\Omega_{\varepsilon}\right) .
$$

We claim that

$$
\left(\left(\nabla \varphi_{\varepsilon}\right)_{0}\right)_{\left.\right|_{\Omega}} \rightarrow \nabla \varphi, \text { in } L^{2}(\Omega),
$$

as $\varepsilon \rightarrow 0$. Indeed, using $\varphi_{\varepsilon}$ as a test function in (160) and the Poincaré inequality we obtain

$$
\left\|\nabla \varphi_{\varepsilon}\right\|_{L^{2}\left(\Omega_{\varepsilon}\right)}^{2} \leq\|\Delta \varphi\|_{L^{2}(\Omega)}\left\|\varphi_{\varepsilon}\right\|_{L^{2}\left(\Omega_{\varepsilon}\right)} \leq c_{N}\left|\Omega_{\varepsilon}\right|^{\frac{1}{N}}\|\Delta \varphi\|_{L^{2}(\Omega)}\left\|\nabla \varphi_{\varepsilon}\right\|_{L^{2}\left(\Omega_{\varepsilon}\right)}
$$

by which we deduce that $\left\|\nabla \varphi_{\varepsilon}\right\|_{L^{2}\left(\Omega_{\varepsilon}\right)}$ is uniformly bounded for $\varepsilon>0$ sufficiently small. Thus, possibly passing to a subsequence, it turns out that $\left(\varphi_{\varepsilon}\right)_{0}$ is strongly convergent in $L^{2}(D)$ and weakly in $H^{1}(D)$ to a function $\psi$. As above, the compact convergence of $\Omega_{\varepsilon}$ implies that $\psi=0$ on $\mathbb{R}^{N} \backslash \bar{\Omega}$. Thus, since $\Omega$ is stable, $\psi \in H_{0}^{1}(\Omega)$. Fixing now $\eta \in C_{c}^{\infty}(\Omega)$ and observing that $\eta \in C_{c}^{\infty}\left(\Omega_{\varepsilon}\right)$ for all $\varepsilon>0$ sufficiently small, we can test $\eta$ in (160) and pass to the limit to conclude that $\psi=\varphi$ in $\Omega$. Thus (161) holds.

Before proceeding with using function $\varphi_{\varepsilon}$, we observe that by assumptions (152) we have that

$$
\int_{\Omega_{\varepsilon} \backslash \Omega}\left|f_{\varepsilon}\right|^{2} d x, \quad \int_{\Omega_{\varepsilon} \backslash \Omega}\left|\nabla f_{\varepsilon}\right|^{2} d x \rightarrow 0
$$

as $\varepsilon \rightarrow 0$, and by the compact convergence of domains we have

$$
\left|\Omega \backslash \Omega_{\varepsilon}\right| \rightarrow 0, \quad \text { as } \varepsilon \rightarrow 0 .
$$

Testing $\varphi_{\varepsilon}$ in the weak formulation of problem (151) we have

$$
\begin{aligned}
\int_{\Omega}\left(\Delta u_{\varepsilon}\right)_{0} \Delta \varphi d x=\int_{\Omega \cap \Omega_{\varepsilon}} \Delta u_{\varepsilon} \Delta \varphi d x=\int_{\Omega_{\Omega} \Omega_{\varepsilon}} \Delta u_{\varepsilon}(\Delta \varphi)_{0} d x \\
=\int_{\Omega_{\varepsilon}} \Delta u_{\varepsilon} \Delta \varphi_{\varepsilon} d x=\int_{\Omega_{\varepsilon}}\left(f_{\varepsilon} \Delta \varphi_{\varepsilon}+\nabla f_{\varepsilon} \nabla \varphi_{\varepsilon}\right) d x \\
=\int_{\Omega_{\varepsilon}}\left(f_{\varepsilon}(\Delta \varphi)_{0}+\nabla f_{\varepsilon} \nabla \varphi_{\varepsilon}\right) d x=\int_{\Omega_{\cap} \Omega_{\varepsilon}}\left(f_{\varepsilon} \Delta \varphi+\nabla f_{\varepsilon} \nabla \varphi_{\varepsilon}\right) d x \\
\quad+\int_{\Omega_{\varepsilon} \backslash \Omega}\left(\nabla f_{\varepsilon} \nabla \varphi_{\varepsilon}\right) d x .
\end{aligned}
$$

Now we note that by the triangle inequality

$$
\begin{aligned}
& \left|\int_{\Omega_{\varepsilon} \cap \Omega} \nabla f_{\varepsilon} \nabla \varphi_{\varepsilon} d x-\int_{\Omega_{\varepsilon} \cap \Omega} \nabla f \nabla \varphi d x\right| \\
& \quad \leq C\left\|\nabla f_{\varepsilon}-\nabla f\right\|_{L^{2}\left(\Omega_{\varepsilon} \cap \Omega\right)}+\left|\int_{\Omega_{\varepsilon} \cap \Omega} \nabla f\left(\nabla \varphi_{\varepsilon}-\nabla \varphi\right) d x\right| .
\end{aligned}
$$

By combining (152), 1161, (163) and (166), it follows that

$$
\int_{\Omega_{\varepsilon} \cap \Omega} \nabla f_{\varepsilon} \nabla \varphi_{\varepsilon} d x=\int_{\Omega_{\varepsilon} \cap \Omega} \nabla f \nabla \varphi d x+o(1), \quad \int_{\Omega_{\cap} \Omega_{\varepsilon}} f_{\varepsilon} \Delta \varphi d x=\int_{\Omega_{\cap} \Omega_{\varepsilon}} f \Delta \varphi d x+o(1),
$$

and $\int_{\Omega_{\varepsilon} \backslash \Omega} \nabla f_{\varepsilon} \nabla \varphi_{\varepsilon} d x \rightarrow 0$, as $\varepsilon \rightarrow 0$. Thus, (165) can be re-written as follows

$$
\int_{\Omega}\left(\Delta u_{\varepsilon}\right)_{0} \Delta \varphi d x=\int_{\Omega \cap \Omega_{\varepsilon}}(f \Delta \varphi+\nabla f \nabla \varphi) d x+o(1) .
$$

By passing to the limit in (168) and using (158), (164) we conclude that $u$ is the solution to problem (147) as required in the statement.

Now, the first limit in (153) follows by the first limit in (157) and by observing that $u$ vanishes almost everywhere outside $\Omega$. 
We now prove that the third limit in (153) holds. To do so, we consider the identity

$$
\begin{aligned}
\left\|\Delta u_{\varepsilon}-(\Delta u)_{0}\right\|_{L^{2}\left(\Omega_{\varepsilon}\right)}^{2} & =\left\|\Delta u_{\varepsilon}\right\|_{L^{2}\left(\Omega_{\varepsilon}\right)}^{2}-2\left(\Delta u_{\varepsilon}, \Delta u\right)_{L^{2}\left(\Omega_{\varepsilon} \cap \Omega\right)}+\|\Delta u\|_{L^{2}\left(\Omega_{\varepsilon} \cap \Omega\right)}^{2} \\
& =\left\|\Delta u_{\varepsilon}\right\|_{L^{2}\left(\Omega_{\varepsilon}\right)}^{2}-2\left(\Delta u_{\varepsilon}, \Delta u\right)_{L^{2}(\Omega)}+\|\Delta u\|_{L^{2}(\Omega)}^{2}+o(1),
\end{aligned}
$$

where the second equality in (169) is deduced by (164).

We note that arguing as above we can prove that

$$
\int_{\Omega_{\varepsilon}}\left(f_{\varepsilon} \Delta u_{\varepsilon}+\nabla f_{\varepsilon} \nabla u_{\varepsilon}\right) d x \rightarrow \int_{\Omega}(f \Delta u+\nabla f \nabla u) d x
$$

as $\varepsilon \rightarrow 0$. By passing to the limit in (155) as $\varepsilon \rightarrow 0$, and by (170), we deduce that

$$
\left\|\Delta u_{\varepsilon}\right\|_{L^{2}\left(\Omega_{\varepsilon}\right)} \rightarrow\|\Delta u\|_{L^{2}(\Omega)}
$$

as $\varepsilon \rightarrow 0$. Finally, using (171) and passing to the limit in (169) allows to conclude that the third limit in (153) holds.

It remains to prove that the second limit in (153) holds. To do so, it is convenient to set $F_{\varepsilon}:=-\Delta u_{\varepsilon}$ and $F:=-\Delta u$. Thus, $u_{\varepsilon}$ and $u$ are solutions to two Poisson problems for the Dirichlet Laplacian in $\Omega_{\varepsilon}$ and $\Omega$ with data $F_{\varepsilon}$ and $F$ respectively, and $\left\|F_{\varepsilon}-F_{0}\right\|_{L^{2}\left(\Omega_{\varepsilon}\right)} \rightarrow 0$ as $\varepsilon \rightarrow 0$. Proceeding as above, using the identity

$$
\left\|\nabla u_{\varepsilon}-(\nabla u)_{0}\right\|_{L^{2}\left(\Omega_{\varepsilon}\right)}^{2}=\left\|\nabla u_{\varepsilon}\right\|_{L^{2}\left(\Omega_{\varepsilon}\right)}^{2}-2\left(\nabla u_{\varepsilon}, \nabla u\right)_{L^{2}(\Omega)}+\|\nabla u\|_{L^{2}(\Omega)}^{2}+o(1)
$$

and exploiting the weak formulations of the Poisson problems for the Dirichlet Laplacian and the weak convergence of the gradients of $\nabla u_{\varepsilon}$, we conclude that the second limit in (153) holds.

5.2. Stability of the Navier-to-Neumann map. It is well-known that if $\Omega$ is a bounded domain of class $C^{0,1}$, given a function $u \in H(\Delta, \Omega) \cap H_{0}^{1}(\Omega)$, it is possible to define the normal derivative $u_{\nu}$ of $u$ as an element of $H^{-1 / 2}(\partial \Omega)$ by setting

$$
H^{-1 / 2(\partial \Omega)}\left\langle u_{\nu}, \varphi\right\rangle_{H^{1 / 2}(\partial \Omega)}=\int_{\Omega}(\Delta u \varphi+\nabla u \nabla \varphi) d x
$$

for all $\varphi \in H^{1 / 2}(\partial \Omega)$, where it is meant that $\varphi$ is extended to the whole of $\Omega$ as an element of $H^{1}(\Omega)$. Recall that the trace operator acts from $H^{1}(\Omega)$ onto $H^{1 / 2}(\partial \Omega)$ and note that definition (173) does not depend on the specific extension of $\varphi$ since the above integral vanishes for functions $\varphi$ belonging to $H_{0}^{1}(\Omega)$.

Since we aim at studying a domain perturbation problem, we find it convenient to consider $u_{\nu}$ as an element of the dual of $H^{1}(\Omega)$ defined by formula (173) for all $\varphi \in H^{1}(\Omega)$.

We call Navier-to-Neumann map the function $\mathcal{N}_{\Omega}$ from $H^{1}(\Omega)$ to $\left(H^{1}(\Omega)\right)^{\prime}$ defined by $\mathcal{N}_{\Omega}(f)=u_{\nu}$ where $u$ is the solution to (147).

We note that if $\Omega$ is sufficiently smooth, say $\Omega$ is a domain of class $C^{1,1}, d$ is an eigenvalue of (2) with eigenfunction $u \in V(\Omega)$ if and only

$$
\mathcal{N}_{\Omega} f=\mu J_{0} f
$$

where $\mu=\frac{1}{d}$ and $f$ is any function in $H^{1}(\Omega)$ such that $f_{\mid \partial \Omega}=d u_{\nu} \in H^{1 / 2}(\partial \Omega)$ and $J_{0}: H^{1}(\Omega) \rightarrow$ $\left(H^{1}(\Omega)\right)^{\prime}$ is defined by

$$
\left(H^{1}(\Omega)\right)^{\prime}\left\langle J_{0} f, v\right\rangle_{H^{1}(\Omega)}:=\int_{\partial \Omega} f v d S \quad \text { for any } f, v \in H^{1}(\Omega) .
$$

Remark 6. If one wishes to consider equation (174) as an eigenvalue problem, it would be clearly appropriate to formulate it on $H^{1}(\Omega) / H_{0}^{1}(\Omega)$ by considering $\mathcal{N}_{\Omega}$ and $J_{0}$ as functions from $H^{1}(\Omega) / H_{0}^{1}(\Omega)$ to its dual. Indeed, any function $f \in H_{0}^{1}(\Omega)$ satisfies (174) for any $\mu \in \mathbb{R}$. However, as we have mentioned in the introduction, using the quotient space would not bypass the main obstructions to the proof of the spectral stability of the classical DBS problem under weak conditions on the convergence of the domains. For this reason, we prefer to work directly on the space $H^{1}(\Omega)$ to avoid further technical complications. 
In Theorems 5.6, 5.7 below we establish convergence results for the family of maps $\mathcal{N}_{\Omega_{\varepsilon}}$ associated with a family of domains $\Omega_{\varepsilon}$ compactly convergent to $\Omega$. For the sake of simplicity, in order to avoid imposing extra assumptions on $\Omega_{\varepsilon}$ or further technicalities in the proofs, we assume that $\Omega \subseteq \Omega_{\varepsilon} \subset D$ for any $\varepsilon>0$, where $D$ is a fixed bounded domain.

Since $\mathcal{N}_{\Omega_{\varepsilon}}$ takes values in the dual of $H^{1}\left(\Omega_{\varepsilon}\right)$, in order to establish the vicinity of $\mathcal{N}_{\Omega_{\varepsilon}}$ to $\mathcal{N}_{\Omega}$ we introduce a notion of $E$-convergence for families of functionals on $H^{1}$.

Definition 5.4. Let $\Omega, \Omega_{\varepsilon}$, with $\varepsilon>0$, be bounded domains in $\mathbb{R}^{N}$ with $\Omega$ of class $C^{0,1}$ and $\Omega \subseteq \Omega_{\varepsilon}$ for all $\varepsilon>0$. Let $\mathcal{E}: H^{1}(\Omega) \rightarrow H^{1}\left(\mathbb{R}^{N}\right)$ be a fixed linear continuous extension operator.

(i) Let $\left\{f_{\varepsilon}\right\}_{\varepsilon>0}$ be a family of functions $f_{\varepsilon} \in H^{1}\left(\Omega_{\varepsilon}\right)$. We say that $f_{\varepsilon} \stackrel{E}{\longrightarrow} f$ with $f \in H^{1}(\Omega)$ if $\left\|f_{\varepsilon}-\mathcal{E} f\right\|_{H^{1}\left(\Omega_{\varepsilon}\right)} \rightarrow 0$ as $\varepsilon \rightarrow 0$.

(ii) Let $\{\Lambda\}_{\varepsilon>0}$ be a family of functionals $\Lambda_{\varepsilon} \in\left(H^{1}\left(\Omega_{\varepsilon}\right)\right)^{\prime}$. We say that $\Lambda_{\varepsilon} \stackrel{E^{*}}{\longrightarrow} \Lambda$ with $\Lambda \in\left(H^{1}(\Omega)\right)^{\prime}$ if

$$
\left\|\Lambda_{\varepsilon}-E_{\varepsilon}^{*} \Lambda\right\|_{\left(H^{1}(\Omega)\right)^{\prime}} \rightarrow 0 \quad \text { as } \varepsilon \rightarrow 0
$$

where $E_{\varepsilon}^{*}:\left(H^{1}(\Omega)\right)^{\prime} \rightarrow\left(H^{1}\left(\Omega_{\varepsilon}\right)\right)^{\prime}$ is the family of linear continuous operators defined by

$$
\left(H^{1}\left(\Omega_{\varepsilon}\right)\right)^{\prime}\left\langle E_{\varepsilon}^{*} \Lambda, v\right\rangle_{H^{1}\left(\Omega_{\varepsilon}\right)}:={ }_{\left(H^{1}(\Omega)\right)^{\prime}}\left\langle\Lambda, v_{\mid \Omega}\right\rangle_{H^{1}(\Omega)} \quad \text { for any } v \in H^{1}\left(\Omega_{\varepsilon}\right)
$$

for any $\Lambda \in\left(H^{1}(\Omega)\right)^{\prime}$.

(iii) Let $\left\{B_{\varepsilon}\right\}_{\varepsilon>0}$ be a family of linear continuous operators such that $B_{\varepsilon}: H^{1}\left(\Omega_{\varepsilon}\right) \rightarrow\left(H^{1}\left(\Omega_{\varepsilon}\right)\right)^{\prime}$. We say that $B_{\varepsilon} \stackrel{E E^{*}}{\longrightarrow} B$ as $\varepsilon \rightarrow 0$, with $B \in \mathcal{L}\left(H^{1}(\Omega) ;\left(H^{1}(\Omega)\right)^{\prime}\right)$, if $B_{\varepsilon} f_{\varepsilon} \stackrel{E^{*}}{\longrightarrow} B f$ whenever $f_{\varepsilon} \stackrel{E}{\longrightarrow} f$.

Remark 7. The family of operators $\left\{E_{\varepsilon}^{*}\right\}_{\varepsilon>0}$ represents a "connecting system" in the sense of [38, Section 1]. In order to see this, one can first show that $\left\|E_{\varepsilon}^{*} \Lambda\right\|_{\left(H^{1}\left(\Omega_{\varepsilon}\right)\right)^{\prime}} \leq\|\Lambda\|_{\left(H^{1}(\Omega)\right)^{\prime}}$ and then, by using the extension operator $\mathcal{E}$ defined above and the fact that $\left|\Omega_{\varepsilon} \backslash \Omega\right| \rightarrow 0$ as $\varepsilon \rightarrow 0$, one shows that for any $\sigma>0$ there exists $\bar{\varepsilon}>0$ such that for any $\varepsilon \in(0, \bar{\varepsilon})$ one has $\|\Lambda\|_{\left(H^{1}(\Omega)\right)^{\prime}} \leq(1+\sigma)\left\|E_{\varepsilon}^{*} \Lambda\right\|_{\left(H^{1}\left(\Omega_{\varepsilon}\right)\right)^{\prime}}+\sigma$; the conclusion follows by letting $\varepsilon \rightarrow 0$ and by exploiting the arbitrariness of $\sigma>0$.

As a consequence of Theorem 5.3 we have

Lemma 5.5. Let $\Omega_{\varepsilon}, \Omega$ and $D$ be as in Theorem 5.3 Assume that $\Omega$ is of class $C^{0,1}, \Omega \subseteq \Omega_{\varepsilon}$ for all $\varepsilon>0$ and $\Omega_{\varepsilon}$ compactly converges to $\Omega$ as $\varepsilon \rightarrow 0$. Let $f_{\varepsilon} \in H^{1}\left(\Omega_{\varepsilon}\right)$ for any $\varepsilon>0$ and $f \in H^{1}(\Omega)$. Let $u_{\varepsilon}$, $u$ be the solutions of problems (151), (147) respectively. If $f_{\varepsilon} \stackrel{E}{\longrightarrow} f$ as $\varepsilon \rightarrow 0$ as above then (153) holds.

Proof. Under the assumptions of the lemma we have that $\left|\Omega_{\varepsilon} \backslash \Omega\right|=\left|\Omega_{\varepsilon} \backslash \bar{\Omega}\right| \rightarrow 0$ as $\varepsilon \rightarrow 0$ hence (152) holds true and Theorem 5.3 applies.

Thanks to Lemma 5.5 we can prove the following

Theorem 5.6. Let $\Omega_{\varepsilon}, \Omega$ and $D$ be as in Theorem 5.3 Assume that $\Omega$ is of class $C^{0,1}, \Omega \subseteq \Omega_{\varepsilon}$ for all $\varepsilon>0$ and $\Omega_{\varepsilon}$ compactly converges to $\Omega$ as $\varepsilon \rightarrow 0$. Let $\mathcal{N}_{\Omega_{\varepsilon}}$ and $\mathcal{N}_{\Omega}$ be the Navier-to-Neumann maps corresponding to $\Omega_{\varepsilon}$ and $\Omega$ respectively. Then $\mathcal{N}_{\Omega_{\varepsilon}} \stackrel{E E^{*}}{\longrightarrow} \mathcal{N}_{\Omega}$ as $\varepsilon \rightarrow 0$.

Proof. We have to prove that

$$
\left\|\mathcal{N}_{\Omega_{\varepsilon}} f_{\varepsilon}-E_{\varepsilon}^{*} \mathcal{N}_{\Omega} f\right\|_{\left(H^{1}\left(\Omega_{\varepsilon}\right)\right)^{\prime}} \rightarrow 0 \quad \text { as } \varepsilon \rightarrow 0
$$

whenever $f_{\varepsilon} \stackrel{E}{\longrightarrow} f$. To do this, we define $u_{\varepsilon}$ and $u$ respectively as the solutions of (151) and (147) and we observe that for any $v \in H^{1}\left(\Omega_{\varepsilon}\right)$ we have

$$
\begin{aligned}
& \left(H^{1}\left(\Omega_{\varepsilon}\right)\right)^{\prime}\left\langle\mathcal{N}_{\Omega_{\varepsilon}} f_{\varepsilon}-E_{\varepsilon}^{*} \mathcal{N}_{\Omega} f, v\right\rangle_{H^{1}\left(\Omega_{\varepsilon}\right)}=\left(H^{1}\left(\Omega_{\varepsilon}\right)\right)^{\prime}\left\langle\left(u_{\varepsilon}\right)_{\nu}, v\right\rangle_{H^{1}\left(\Omega_{\varepsilon}\right)}-{ }_{\left(H^{1}(\Omega)\right)^{\prime}}\left\langle u_{\nu}, v_{\mid \Omega}\right\rangle_{H^{1}(\Omega)} \\
& \quad=\int_{\Omega_{\varepsilon}}\left(\Delta u_{\varepsilon} v+\nabla u_{\varepsilon} \nabla v\right) d x-\int_{\Omega}(\Delta u v+\nabla u \nabla v) d x \\
& =\int_{\Omega}\left[\left(\Delta u_{\varepsilon}-\Delta u\right) v+\left(\nabla u_{\varepsilon}-\nabla u\right) \nabla v\right] d x+\int_{\Omega_{\varepsilon} \backslash \Omega}\left(\Delta u_{\varepsilon} v+\nabla u_{\varepsilon} \nabla v\right) d x .
\end{aligned}
$$


This implies

(177)

$$
\begin{aligned}
& \left|\left(H^{1}\left(\Omega_{\varepsilon}\right)\right)^{\prime}\left\langle\mathcal{N}_{\Omega_{\varepsilon}} f_{\varepsilon}-E_{\varepsilon}^{*} \mathcal{N}_{\Omega} f, v\right\rangle_{H^{1}\left(\Omega_{\varepsilon}\right)}\right| \\
& \quad \leq\left[\left\|\Delta u_{\varepsilon}-\Delta u\right\|_{L^{2}(\Omega)}+\left\|\nabla u_{\varepsilon}-\nabla u\right\|_{L^{2}(\Omega)}+\left\|\Delta u_{\varepsilon}\right\|_{L^{2}\left(\Omega_{\varepsilon} \backslash \Omega\right)}+\left\|\nabla u_{\varepsilon}\right\|_{L^{2}\left(\Omega_{\varepsilon} \backslash \Omega\right)}\right]\|v\|_{H^{1}\left(\Omega_{\varepsilon}\right)} .
\end{aligned}
$$

The proof of (176) then follows from Lemma 5.5 , This completes the proof of the lemma.

Finally we prove the compact convergence $\mathcal{N}_{\Omega_{\varepsilon}} \stackrel{C^{*}}{\longrightarrow} \mathcal{N}_{\Omega}$, i.e. $\mathcal{N}_{\Omega_{\varepsilon}} \stackrel{E E^{*}}{\longrightarrow} \mathcal{N}_{\Omega}$ and $\left\{\mathcal{N}_{\Omega_{\varepsilon}} f_{\varepsilon}\right\}_{\varepsilon>0}$ is precompact in the sense of Definition 2.3 with $\mathcal{H}_{\varepsilon}=\left(H^{1}\left(\Omega_{\varepsilon}\right)\right)^{\prime}$ and $\mathcal{H}_{0}=\left(H^{1}(\Omega)\right)^{\prime}$ whenever $\left\|f_{\varepsilon}\right\|_{H^{1}\left(\Omega_{\varepsilon}\right)}=1$. To do so, we shall require that the following condition (A) is satisfied.

(A): For any family $\left\{f_{\varepsilon}\right\}_{0<\varepsilon \leq \varepsilon_{0}}$ of functions $f_{\varepsilon} \in H^{1}\left(\Omega_{\varepsilon}\right)$ such that $\left\|f_{\varepsilon}\right\|_{H^{1}\left(\Omega_{\varepsilon}\right)}$ is uniformly bounded in $\varepsilon$, we have that $\left\|f_{\varepsilon}\right\|_{L^{2}\left(\Omega_{\varepsilon} \backslash \Omega\right)} \rightarrow 0$ as $\varepsilon \rightarrow 0$.

This condition has been extensively used by J.M. Arrieta and his co-authors in the analysis of the spectral stability of second order elliptic operators subject to Neumann boundary conditions on dumbbell domains, see [3] for references. We note that in our case, this condition will be used also to control the behaviour of the data $f_{\varepsilon}$ of the problems.

Remark 8. If $\Omega_{\varepsilon}, \Omega$ are of class $C^{0}(\mathcal{A})$ for all $\varepsilon$, where $\mathcal{A}$ is a fixed atlas, then the compact convergence of $\Omega_{\varepsilon}$ to $\Omega$ is equivalent to the uniform convergence of the functions describing the boundaries of $\Omega_{\varepsilon}$ and that using the boundedness of functions in Sobolev spaces along lines as in Lemma 3.1 we have that the compact convergence implies the validity of condition $(A)$.

Theorem 5.7. Let $\Omega_{\varepsilon}, \Omega$ and $D$ be as in Theorem 5.3 Assume that $\Omega$ is of class $C^{0,1}, \Omega \subseteq \Omega_{\varepsilon}$ for all $\varepsilon>0, \Omega_{\varepsilon}$ compactly converges to $\Omega$ as $\varepsilon \rightarrow 0$, and condition $(A)$ is satisfied. Let $\mathcal{N}_{\Omega_{\varepsilon}}$ and $\mathcal{N}_{\Omega}$ be the Navier-to-Neumann maps corresponding to $\Omega_{\varepsilon}$ and $\Omega$ respectively. Then $\mathcal{N}_{\Omega_{\varepsilon}} \stackrel{C^{*}}{\longrightarrow} \mathcal{N}_{\Omega}$ as $\varepsilon \rightarrow 0$.

Proof. Let $f_{\varepsilon} \in H^{1}\left(\Omega_{\varepsilon}\right)$ be such that $\left\|f_{\varepsilon}\right\|_{H^{1}\left(\Omega_{\varepsilon}\right)}=1$ for all $\varepsilon>0$. Let $u_{\varepsilon} \in H\left(\Delta, \Omega_{\varepsilon}\right) \cap H_{0}^{1}\left(\Omega_{\varepsilon}\right)$ be the solutions to problem (151). Proceeding as in the proof of Theorem 5.3 one can show that possibly passing to a subsequence (156), (157), (158) hold true for some $u \in H^{1}(D)$ such that $u_{\mid \Omega} \in H(\Delta, \Omega) \cap H_{0}^{1}(\Omega), u$ vanishing outside $\Omega$. Writing

$$
\int_{\Omega_{\varepsilon}}\left|\nabla u_{\varepsilon}\right|^{2} d x=\int_{\Omega_{\varepsilon}}-u_{\varepsilon} \Delta u_{\varepsilon} d x=\int_{\Omega}-u_{\varepsilon} \Delta u_{\varepsilon} d x+\int_{\Omega_{\varepsilon} \backslash \Omega}-u_{\varepsilon} \Delta u_{\varepsilon} d x
$$

and passing to the limit as $\varepsilon \rightarrow 0$, we have by 156, 1157, , 158) and condition $(A)$

$$
\lim _{\varepsilon \rightarrow 0} \int_{\Omega_{\varepsilon}}\left|\nabla u_{\varepsilon}\right|^{2} d x=\lim _{\varepsilon \rightarrow 0} \int_{\Omega}-u_{\varepsilon} \Delta u_{\varepsilon} d x=\int_{\Omega}-u \Delta u d x=\int_{\Omega}|\nabla u|^{2} d x .
$$

Moreover,

$$
\int_{\Omega}|\nabla u|^{2} d x \leq \liminf _{\varepsilon \rightarrow 0} \int_{\Omega}\left|\nabla u_{\varepsilon}\right|^{2} d x \leq \limsup _{\varepsilon \rightarrow 0} \int_{\Omega}\left|\nabla u_{\varepsilon}\right|^{2} d x \leq \lim _{\varepsilon \rightarrow 0} \int_{\Omega_{\varepsilon}}\left|\nabla u_{\varepsilon}\right|^{2} d x=\int_{\Omega}|\nabla u|^{2} d x
$$

hence

$$
\int_{\Omega}\left|\nabla u_{\varepsilon}\right|^{2} d x \rightarrow \int_{\Omega}|\nabla u|^{2} d x, \quad \int_{\Omega_{\varepsilon} \backslash \Omega}\left|\nabla u_{\varepsilon}\right|^{2} d x \rightarrow 0, \quad\left(u_{\varepsilon}\right)_{\mid \Omega} \rightarrow u \quad \text { in } H^{1}(\Omega),
$$

as $\varepsilon \rightarrow 0$.

By our assumptions, in particular by condition $(A)$, we have that possibly passing to a subsequence there exists $f \in H^{1}(\Omega)$ such that

$$
f_{\varepsilon} \rightarrow f \quad \text { in } L^{2}(\Omega), \quad f_{\varepsilon} \rightarrow f \quad \text { in } H^{1}(\Omega), \quad\left\|f_{\varepsilon}\right\|_{L^{2}\left(\Omega_{\varepsilon} \backslash \Omega\right)} \rightarrow 0
$$

as $\varepsilon \rightarrow 0$. We claim that function $u$ is a solution to problem (147) with this datum $f$. To prove this, one can use the same argument as in the proof of Theorem 5.3. Here we cannot use the strong convergence of the 
gradients of $f_{\varepsilon}$, not even the second limit in (163). However, by looking closely at the proof of Theorem 5.3, one can see that here it suffices to use the fact that the family of functions $\varphi_{\varepsilon}$ used in that proof satisfies the conditions $\varphi_{\varepsilon} \rightarrow \varphi$ in $H^{1}(\Omega)$ and $\left\|\nabla \varphi_{\varepsilon}\right\|_{L^{2}\left(\Omega_{\varepsilon} \backslash \Omega\right)} \rightarrow 0$ as $\varepsilon \rightarrow 0$, which can be proved by proceeding exactly in the same way as in (178)-(181).

By (156), 158), (181), (182), we conclude that

$$
\begin{gathered}
\int_{\Omega_{\varepsilon}}\left|\Delta u_{\varepsilon}\right|^{2} d x=\int_{\Omega}\left(f_{\varepsilon} \Delta u_{\varepsilon}+\nabla f_{\varepsilon} \nabla u_{\varepsilon}\right) d x+\int_{\Omega_{\varepsilon} \backslash \Omega}\left(f_{\varepsilon} \Delta u_{\varepsilon}+\nabla f_{\varepsilon} \nabla u_{\varepsilon}\right) d x \\
=\int_{\Omega}(f \Delta u+\nabla f \nabla u) d x+o(1)=\int_{\Omega}|\Delta u|^{2} d x+o(1) .
\end{gathered}
$$

In particular, proceeding as we have done above for $\nabla u_{\varepsilon}$, we have that

$$
\left(\Delta u_{\varepsilon}\right)_{\mid \Omega} \rightarrow \Delta u \quad \text { in } L^{2}(\Omega), \quad \int_{\Omega_{\varepsilon} \backslash \Omega}\left|\Delta u_{\varepsilon}\right|^{2} d x \rightarrow 0
$$

as $\varepsilon \rightarrow 0$. Inserting (181) and (183) into 1777) we obtain $\mathcal{N}_{\Omega_{\varepsilon}} f_{\varepsilon} \stackrel{E^{*}}{\rightarrow} \mathcal{N}_{\Omega} f$ as $\varepsilon \rightarrow 0$ along a sequence. We have proved that $\left\{\mathcal{N}_{\Omega_{\varepsilon}} f_{\varepsilon}\right\}$ is precompact in the sense of Definition 2.3. The proof of the theorem now follows combining this with Theorem 5.6 .

Unfortunately, as we have mentioned in the introduction the compact convergence result obtained in Theorem 5.7 is not sufficient to prove a stability result for the spectrum of the Steklov problem (2). Thus we suggest the following open problem

Open Problem 5.1. Let $\left\{\Omega_{\varepsilon}\right\}_{0<\varepsilon \leq \varepsilon_{0}}$ and $\Omega$ be such that condition (21) is not satisfied. For simplicity one may think to a family of domains as in Section 4 and choose $1 \leq \alpha \leq \frac{3}{2}$ is such a way that (21) is not satisfied. Study the stability of the spectrum of (2).

We recall that, in a situation like the one proposed in Open Problem 5.1, we proved that the spectrum of the modified Steklov problem (5) does not behave continuously as $\varepsilon \rightarrow 0$. The question proposed in Open Problem 5.1 aims at clarifying what happens to the spectrum of (2) under the very same assumptions.

5.3. Instability of a modified Navier problem. Given a sufficiently smooth bounded domain $\Omega$ in $\mathbb{R}^{N}$ we consider the boundary value problem

$$
\begin{cases}\Delta^{2} u=0, & \text { in } \Omega, \\ u=0, & \text { on } \partial \Omega, \\ \Delta u-K(x) u_{\nu}=f, & \text { on } \partial \Omega,\end{cases}
$$

in the unknown $u$. The natural way of writing the weak formulation of 184 is

$$
\int_{\Omega} D^{2} u: D^{2} \varphi d x=\int_{\partial \Omega} f \varphi_{\nu} d S, \text { for all } \varphi \in H^{2}(\Omega) \cap H_{0}^{1}(\Omega)
$$

where the unknown $u$ has to be considered in $H^{2}(\Omega) \cap H_{0}^{1}(\Omega)$. This formulation allows to consider the datum $f$ in $L^{2}(\partial \Omega)$. However, in order to emphasize the peculiar behaviour of this problem with respect to problem (149), we prefer to write (185) in a form similar to (149). Thus we assume that $f$ is the trace of function $f \in H^{1}(\Omega)$ and rewrite (185) as

$$
\int_{\Omega} D^{2} u: D^{2} \varphi d x=\int_{\Omega}(f \Delta \varphi+\nabla f \nabla \varphi) d x, \text { for all } \varphi \in H^{2}(\Omega) \cap H_{0}^{1}(\Omega) .
$$


As in the previous section, we now consider a family of domains $\Omega_{\varepsilon}, \varepsilon>0$ and the corresponding boundary value problems

$$
\begin{cases}\Delta^{2} u_{\varepsilon}=0, & \text { in } \Omega_{\varepsilon}, \\ u_{\varepsilon}=0, & \text { on } \partial \Omega_{\varepsilon}, \\ \Delta u_{\varepsilon}-K_{\varepsilon}(x)\left(u_{\varepsilon}\right)_{\nu}=f_{\varepsilon}, & \text { on } \partial \Omega_{\varepsilon},\end{cases}
$$

in the unknown $u_{\varepsilon} \in H^{2}\left(\Omega_{\varepsilon}\right) \cap H_{0}^{1}(\Omega)$, where $f_{\varepsilon} \in H^{1}\left(\Omega_{\varepsilon}\right)$, with the understanding that problem (187) has to be interpreted as in (186).

We assume that $\Omega$ and $\Omega_{\varepsilon}$ are as in Section 4, As in [4], the limiting behaviour of problem (187) depends on the value of $\alpha$. In particular, we have stability for $\alpha>3 / 2$ and degeneration for $\alpha<3 / 2$. For $\alpha=3 / 2$, the limiting problem involves the strange factor $\gamma$ defined by (134).

Remark 9. Theorem 5.8 below and Theorem 5.3 show that problem (147) is much more stable than problem (184) under domain perturbations. Note that the main step in the proof of Theorem 5.3 is the definition of the test function $\varphi_{\varepsilon}$ in (159). Unless the convergence of the domains $\Omega_{\varepsilon}$ to $\Omega$ is stronger, this function cannot be used in the analysis of problem (184), where more information on the Hessian of $\varphi_{\varepsilon}$ would be required.

Theorem 5.8. Assume that $\Omega$ and $\Omega_{\varepsilon}, \varepsilon>0$, are as in Section 4 and that the datum $f_{\varepsilon}$ converges to $f$ in the sense of (152). Let $u_{\varepsilon}$ be the solution of problem (187). Then the following statements hold:

(i) If $\alpha>3 / 2$ then $u_{\varepsilon}$ converges to the solution of problem (184) in the sense that for every multi-index $\beta \in \mathbb{N}_{0}^{N}$ with $0 \leq|\beta| \leq 2$ we have $\left\|D^{\beta} u_{\varepsilon}-\left(D^{\beta} u\right)_{0}\right\|_{L^{2}\left(\Omega_{\varepsilon}\right)} \rightarrow 0$, as $\varepsilon \rightarrow 0$.

(ii) If $\alpha=3 / 2$ then $u_{\varepsilon}$ converges to the solution $u$ of the problem

$$
\begin{cases}\Delta^{2} u=0, & \text { in } \Omega, \\ u=0 \text { and } \Delta u-K(x) u_{\nu}=f, & \text { on } \partial \Omega \backslash \Gamma, \\ u=0 \text { and } \Delta u-K(x) u_{\nu}+\gamma u_{\nu}=f, & \text { on } \Gamma,\end{cases}
$$

in the sense that for every multi-index $\beta \in \mathbb{N}_{0}^{N}$ with $0 \leq|\beta| \leq 1$ we have $\left\|D^{\beta} u_{\varepsilon}-\left(D^{\beta} u\right)_{0}\right\|_{L^{2}\left(\Omega_{\varepsilon}\right)} \rightarrow$ 0 , and $u_{\varepsilon} \rightarrow u$ weakly in $H^{2}(\Omega)$ as $\varepsilon \rightarrow 0$.

(iii) If $0<\alpha<3 / 2$ then $u_{\varepsilon}$ converges to the solution $u$ of the problem

$$
\begin{cases}\Delta^{2} u=0, & \text { in } \Omega, \\ u=0 \text { and } \Delta u-K(x) u_{\nu}=f, & \text { on } \partial \Omega \backslash \Gamma, \\ u=0 \text { and } u_{\nu}=0, & \text { on } \Gamma,\end{cases}
$$

in the sense that for every multi-index $\beta \in \mathbb{N}_{0}^{N}$ with $0 \leq|\beta| \leq 2$ we have $\left\|D^{\beta} u_{\varepsilon}-\left(D^{\beta} u\right)_{0}\right\|_{L^{2}\left(\Omega_{\varepsilon}\right)} \rightarrow$ 0 , as $\varepsilon \rightarrow 0$.

Proof. In each of the three cases, the solution $u_{\varepsilon} \in H^{2}\left(\Omega_{\varepsilon}\right) \cap H_{0}^{1}\left(\Omega_{\varepsilon}\right)$ of problem (187) satisfies the weak equation

$$
\int_{\Omega_{\varepsilon}} D^{2} u_{\varepsilon}: D^{2} \varphi d x=\int_{\Omega_{\varepsilon}}\left(f_{\varepsilon} \Delta \varphi+\nabla f_{\varepsilon} \nabla \varphi\right) d x
$$

for all $\varphi \in H^{2}\left(\Omega_{\varepsilon}\right) \cap H_{0}^{1}\left(\Omega_{\varepsilon}\right)$. Thus, by using $u_{\varepsilon}$ as test function in (190) and arguing as in the proof of Theorem 5.3, we get that $\left\|u_{\varepsilon}\right\|_{H^{2}\left(\Omega_{\varepsilon}\right)}$ is uniformly bounded, hence there exists $u \in H^{2}(\Omega) \cap H_{0}^{1}(\Omega)$ such that possibly passing to a subsequence, $u_{\varepsilon} \rightarrow u$ in $H^{2}(\Omega)$ and $u_{\varepsilon} \rightarrow u$ in $H^{1}(\Omega)$. We have now to identify the limiting problem satisfied by $u$ and this depends on the value of $\alpha$.

We begin with statement (i). For every $\varepsilon>0$ sufficiently small, say $\varepsilon \in] 0, \varepsilon_{0}$ [, we consider a diffeomorphism $\Phi_{\varepsilon}$ from $\bar{\Omega}_{\varepsilon}$ onto $\bar{\Omega}$ defined exactly as the diffeomorphism $\Phi_{\varepsilon, j}$ introduced in Subsection 3.2 . To 
do so, for fixed $\tilde{\alpha} \in] 3 / 2, \alpha$, we set $\kappa_{\varepsilon}=\varepsilon^{2 \tilde{\alpha} / 3}$ and we note that condition (21) is satisfied for $\varepsilon_{0}$ sufficiently small. Then, $\Phi_{\varepsilon}$ is defined by $\Phi_{\varepsilon}\left(x^{\prime}, x_{N}\right)=\left(x^{\prime}, x_{N}-h_{\varepsilon}\left(x^{\prime}, x_{N}\right)\right)$ for all $\left(x^{\prime}, x_{N}\right) \in \bar{\Omega}_{\varepsilon}$ where $h_{\varepsilon}$ is defined as $h_{\varepsilon, j}$ by formula (24) with $g_{\varepsilon, j}$ replaced by $g_{\varepsilon}, g_{j}$ replaced by the zero function, and $a_{N, j}$ replace by -1 . It is also convenient to recall that $\Phi_{\varepsilon}\left(x^{\prime}, x_{N}\right)=\left(x^{\prime}, x_{N}\right)$ for all $\left(x^{\prime}, x_{N}\right) \in K_{\varepsilon}$ where $K_{\varepsilon}=\left\{\left(x^{\prime}, x_{N}\right) \in W \times \mathbb{R}:-1<x_{N}<\tilde{g}_{\varepsilon}\left(x^{\prime}, x_{N}\right)\right\}$ and $\tilde{g}_{\varepsilon}=-k_{\varepsilon}=-\hat{k} \kappa_{\varepsilon}$ with $\hat{k}>6$ as in Subsection 3.2

Let $\varphi \in H^{2}(\Omega) \cap H_{0}^{1}(\Omega)$. We set $\varphi_{\varepsilon}=\varphi \circ \Phi_{\varepsilon}$ and we note that $\varphi_{\varepsilon} \in H^{2}\left(\Omega_{\varepsilon}\right) \cap H_{0}^{1}\left(\Omega_{\varepsilon}\right)$, hence it can be used as test function in (190). Thus, since $\Phi_{\varepsilon}$ coincides with the identity on $K_{\varepsilon}$ we have

$$
\begin{aligned}
\int_{K_{\varepsilon}} D^{2} u_{\varepsilon} & : D^{2} \varphi d x+\int_{\Omega_{\varepsilon} \backslash K_{\varepsilon}} D^{2} u_{\varepsilon}: D^{2} \varphi_{\varepsilon} d x \\
& =\int_{\Omega_{\varepsilon}} D^{2} u_{\varepsilon}: D^{2} \varphi_{\varepsilon} d x=\int_{\Omega_{\varepsilon}}\left(f_{\varepsilon} \Delta \varphi_{\varepsilon}+\nabla f_{\varepsilon} \nabla \varphi_{\varepsilon}\right) d x \\
& =\int_{K_{\varepsilon}}\left(f_{\varepsilon} \Delta \varphi+\nabla f_{\varepsilon} \nabla \varphi\right) d x+\int_{\Omega_{\varepsilon} \backslash K_{\varepsilon}}\left(f_{\varepsilon} \Delta \varphi_{\varepsilon}+\nabla f_{\varepsilon} \nabla \varphi_{\varepsilon}\right) d x .
\end{aligned}
$$

By Lemma 3.1 we have that (20) holds hence $\int_{\Omega_{\varepsilon} \backslash K_{\varepsilon}}\left|D^{2} \varphi_{\varepsilon}\right|^{2} d x \rightarrow 0$ and $\int_{\Omega_{\varepsilon} \backslash K_{\varepsilon}} D^{2} u_{\varepsilon}: D^{2} \varphi_{\varepsilon} d x \rightarrow 0$ as $\varepsilon \rightarrow 0$. By a similar argument, one can also prove that $\int_{\Omega_{\varepsilon} \backslash K_{\varepsilon}}\left(f_{\varepsilon} \Delta \varphi_{\varepsilon}+\nabla f_{\varepsilon} \nabla \varphi_{\varepsilon}\right) d x \rightarrow 0$ as $\varepsilon \rightarrow 0$ (in which case one should use a limiting relation for the norms of the gradients analogous to (20), see the proof of Theorem 5.7 for more details).

Thus, passing to the limit in 191) we obtain

$$
\int_{\Omega} D^{2} u: D^{2} \varphi d x=\int_{\Omega}(f \Delta \varphi+\nabla f \nabla \varphi) d x .
$$

Using the same argument as in the proof of Theorem 5.3 with identity 169 replaced by

$$
\left\|D^{2} u_{\varepsilon}-\left(D^{2} u\right)_{0}\right\|_{L^{2}\left(\Omega_{\varepsilon}\right)}^{2}=\left\|D^{2} u_{\varepsilon}\right\|_{L^{2}\left(\Omega_{\varepsilon}\right)}^{2}-2 \int_{\Omega} D^{2} u_{\varepsilon}: D^{2} u d x+\left\|D^{2} u\right\|_{L^{2}(\Omega)}^{2},
$$

we deduce that $\left\|D^{2} u_{\varepsilon}-\left(D^{2} u\right)_{0}\right\|_{L^{2}\left(\Omega_{\varepsilon}\right)} \rightarrow 0$ as $\varepsilon \rightarrow 0$. In order to conclude, it suffices to use the strong convergence in $H^{1}(\Omega)$ stated above and to note that for any multi-index $|\beta| \leq 1$ we have $\left\|D^{\beta} u_{\varepsilon}\right\|_{L^{2}\left(\Omega_{\varepsilon} \backslash \Omega\right)} \rightarrow$ 0 as $\varepsilon \rightarrow 0$ which follows from the fact that for $|\beta| \leq 1$ and for almost all $x^{\prime} \in W$ we have that $D^{\beta} u_{\varepsilon}\left(x^{\prime}, x_{N}\right)$ is bounded in $x_{N}$, see (34) for more details.

We now prove statement (ii). In this case, we need a different diffeomorphism from $\bar{\Omega}_{\varepsilon}$ onto $\bar{\Omega}$, which for convenience we denote with the same symbol $\Phi_{\varepsilon}$. Namely, $\Phi_{\varepsilon}\left(x^{\prime}, x_{N}\right)=\left(x^{\prime}, x_{N}-h_{\varepsilon}\left(x^{\prime}, x_{N}\right)\right)$ for all $\left(x^{\prime}, x_{N}\right) \in \bar{\Omega}_{\varepsilon}$ where $h_{\varepsilon}$ is defined as in (138).

It is convenient to denote again by $K_{\varepsilon}$ the set where $\Phi_{\varepsilon}$ coincides with the identity, that is $K_{\varepsilon}=\left\{\left(x^{\prime}, x_{N}\right) \in\right.$ $\left.W \times \mathbb{R}:-1<x_{N} \leq-\varepsilon\right\}$. Let $\varphi \in H^{2}(\Omega) \cap H_{0}^{1}(\Omega)$. We set $\varphi_{\varepsilon}=\varphi \circ \Phi_{\varepsilon}$ and we note that $\varphi_{\varepsilon} \in H^{2}\left(\Omega_{\varepsilon}\right) \cap H_{0}^{1}\left(\Omega_{\varepsilon}\right)$, hence it can be used as test function in (190). Clearly, equality (191) holds. Concerning the second term in the right-hand side of (191), we note that

$$
\left|\int_{\Omega_{\varepsilon} \backslash K_{\varepsilon}} f_{\varepsilon} \Delta \varphi_{\varepsilon} d x\right| \leq\left\|f_{\varepsilon}\right\|_{L^{2}\left(\Omega_{\varepsilon} \backslash K_{\varepsilon}\right)}\left\|\Delta \varphi_{\varepsilon}\right\|_{L^{2}\left(\Omega_{\varepsilon} \backslash K_{\varepsilon}\right)}=o(1)\left\|\Delta \varphi_{\varepsilon}\right\|_{L^{2}\left(\Omega_{\varepsilon} \backslash K_{\varepsilon}\right)}
$$

and

$$
\left|\int_{\Omega_{\varepsilon} \backslash K_{\varepsilon}} \nabla f_{\varepsilon} \nabla \varphi_{\varepsilon} d x\right| \leq\left\|\nabla f_{\varepsilon}\right\|_{L^{2}\left(\Omega_{\varepsilon} \backslash K_{\varepsilon}\right)}\left\|\nabla \varphi_{\varepsilon}\right\|_{L^{2}\left(\Omega_{\varepsilon} \backslash K_{\varepsilon}\right)}=o(1)\left\|\nabla \varphi_{\varepsilon}\right\|_{L^{2}\left(\Omega_{\varepsilon} \backslash K_{\varepsilon}\right)}
$$

where we have used (163). On the other hand, one can see that

$$
\left\|D^{2} \varphi_{\varepsilon}\right\|_{L^{2}\left(\Omega_{\varepsilon} \backslash K_{\varepsilon}\right)}=O(1), \text { and }\left\|\nabla \varphi_{\varepsilon}\right\|_{L^{2}\left(\Omega_{\varepsilon} \backslash K_{\varepsilon}\right)}=o(1) .
$$

Indeed, by the chain rule it follows that 


$$
\begin{aligned}
\frac{\partial^{2} \varphi_{\varepsilon}}{\partial x_{n} \partial x_{m}}(x) & =\sum_{k=1}^{N}\left[\sum_{l=1}^{N} \frac{\partial^{2} \varphi}{\partial x_{l} \partial x_{k}}\left(\Phi_{\varepsilon}(x)\right) \frac{\partial\left[\left(\Phi_{\varepsilon}(x)\right)_{l}\right]}{\partial x_{m}}\right] \frac{\partial\left[\left(\Phi_{\varepsilon}(x)\right)_{k}\right]}{\partial x_{n}} \\
& +\frac{\partial \varphi}{\partial x_{k}}\left(\Phi_{\varepsilon}(x)\right) \frac{\partial^{2}\left[\left(\Phi_{\varepsilon}(x)\right)_{k}\right]}{\partial x_{n} \partial x_{m}} .
\end{aligned}
$$

Now the $L^{2}$-norm of the first term in the right-hand side of (197) is clearly $O\left(\varepsilon^{2 \alpha-2}\right)$ hence it is infinitesimal for $\alpha=3 / 2$ as $\varepsilon \rightarrow 0$. As for the second term, we note that $\frac{\partial^{2}\left[\left(\Phi_{\varepsilon}(x)\right)_{k}\right]}{\partial x_{n} \partial x_{m}}=O\left(\varepsilon^{\alpha-2}\right)$ while $\left\|\frac{\partial \varphi_{\varepsilon}}{\partial x_{k}}\left(\Phi_{\varepsilon}(x)\right)\right\|_{L^{2}\left(\Omega \backslash K_{\varepsilon}\right)}=O\left(\varepsilon^{1 / 2}\right)$ which simply follows by the fact that for almost all $x^{\prime} \in W, \frac{\partial \varphi_{\varepsilon}}{\partial x_{k}}\left(x^{\prime}, x_{N}\right)$ is bounded in the variable $x_{N}$. Thus, for $\alpha=3 / 2$ we have that the $L^{2}$-norm of the second term in the right-hand side of (197) is $O(1)$ as $\varepsilon \rightarrow 0$, hence the first equality in (196) is proved. The second equality in (196) can be proved in the same way. The proof of statement (ii) follows by passing to the limit in (191) as $\varepsilon \rightarrow 0$ and using (140), (196).

We now prove statement (iii). It follows from the proof of [5, Theorem 7.4] that since $\alpha<3 / 2$ the function $u$ belongs to $H_{0, \Gamma}^{2}(\Omega) \cap H_{0}^{1}(\Omega)$ where $H_{0, \Gamma}^{2}(\Omega)=\left\{u \in H^{2}(\Omega): u=u_{\nu}=0\right.$ on $\left.\Gamma\right\}$, see the proof of Lemma 4.4 for more details. Let $\varphi \in H_{0, \Gamma}^{2}(\Omega)$. Since $\varphi=\varphi_{\nu}=0$ on $\Gamma$, the extension-by-zero $\varphi_{0}$ of $\varphi$ belongs to $H^{2}\left(\Omega_{\varepsilon}\right) \cap H_{0}^{1}\left(\Omega_{\varepsilon}\right)$, hence it can uses ad test function in (190) to obtain

$$
\int_{\Omega} D^{2} u_{\varepsilon}: D^{2} \varphi d x=\int_{\Omega_{\varepsilon}} D^{2} u_{\varepsilon}: D^{2} \varphi_{0} d x=\int_{\Omega_{\varepsilon}}\left(f_{\varepsilon} \Delta \varphi_{0}+\nabla f_{\varepsilon} \nabla \varphi_{0}\right) d x=\int_{\Omega}\left(f_{\varepsilon} \Delta \varphi+\nabla f_{\varepsilon} \nabla \varphi\right) d x .
$$

Passing to the limit in (198) as $\varepsilon \rightarrow 0$ we conclude that $u$ is the solution to problem (189). The proof of the convergence of $u_{\varepsilon}$ to $u$ in the $H^{2}$ norms follows the same lines of the analogous proof in statement (i).

Acknowledgments The authors are grateful to the anonymous referee for the very careful reading of the paper and for the many useful comments and suggestions which helped them to improve the presentation of the paper, and for bringing to their attention item [33] in the references list. The authors are also very thankful to Professors José M. Arrieta and Giles Auchmuty for useful discussions and references. The authors are members of the Gruppo Nazionale per l'Analisi Matematica, la Probabilità e le loro Applicazioni (GNAMPA) of the Istituto Nazionale di Alta Matematica (INdAM). The first author acknowledges partial financial support from the PRIN project 2012 "Equazioni alle derivate parziali di tipo ellittico e parabolico: aspetti geometrici, disuguaglianze collegate, e applicazioni". The first author acknowledges partial financial support from the INDAM - GNAMPA project 2017 "Stabilità e analisi spettrale per problemi alle derivate parziali". The second author acknowledges partial financial support from the INDAM - GNAMPA project 2017 "Equazioni alle derivate parziali non lineari e disuguaglianze funzionali: aspetti geometrici ed analitici". This research was partially supported by the research project CPDA120171/12 "Singular perturbation problems for differential operators" Progetto di Ateneo of the University of Padova and by the research project "Metodi analitici, numerici e di simulazione per lo studio di equazioni differenziali a derivate parziali e applicazioni" Progetto di Ateneo 2016 of the University of Piemonte Orientale "Amedeo Avogadro".

\section{REFERENCES}

[1] V. Adolfsson, $L^{2}$-integrability of second order derivatives for Poisson's equation in nonsmooth domains, Math. Scand. 70, 146-160, (1992).

[2] P. R. S. Antunes, F. Gazzola, Convex shape optimization for the least biharmonic Steklov eigenvalue, ESAIM COCV 19 , 385-403, (2013).

[3] J. M. Arrieta, A. N. Carvalho, G. Losada-Cruz, Dynamics in dumbell domains I. Continuity of the set of equilibria, J. Diff. Eq. 231, 551-597, (2006).

[4] J. M. Arrieta, P. D. Lamberti, Higher order elliptic operators on variable domains. Stability results and boundary oscillations for intermediate problems, J. Differential Equations 263, no. 7, 4222-4266, (2017). 
[5] J. M. Arrieta, P. D. Lamberti, Spectral stability results for higher order operators under perturbations of the domain, C. R. Acad. Sci. Paris, ser. I 351, 725-730, (2013).

[6] J.M. Arrieta, M. López-Fernández, E. Zuazua, Enrique Approximating travelling waves by equilibria of non-local equations, Asymptot. Anal. 78, no. 3, 145-186, (2012).

[7] G. Auchmuty, The S.V.D. of the Poisson Kernel, J. Fourier Anal. Appl. 23, no. 6, 1517-1536, (2017).

[8] E. Berchio, F. Gazzola, E. Mitidieri, Positivity preserving property for a class of biharmonic elliptic problems, J. Differ. Equa. 229, 1-23, (2006).

[9] S. Bögli, Convergence of sequences of linear operators and their spectra, Integral Equations Operator Theory 88, no. 4, 559-599, (2017).

[10] B. Bogosel, The Steklov Spectrum on Moving Domains, Appl. Math. Optim. 75, 1-25, (2017).

[11] H. Brezis, Analisi funzionale, Liguori Editore, (1986)

[12] D. Bucur, G. Buttazzo, Variational methods in shape optimization problems, Progress in Nonlinear Differential Equations and their Applications 65, Birkhäuser Boston, Boston, (2005).

[13] D. Bucur, A. Ferrero, F. Gazzola, On the first eigenvalue of a fourth order Steklov problem, Calc. Var. PDE 35, 103-131, (2009).

[14] D. Bucur, F. Gazzola, The first biharmonic Steklov eigenvalue: positivity preserving and shape optimization, Milan J. Math. 79, 247-258, (2011).

[15] D. Buoso, L. Provenzano, A few shape optimization results for a biharmonic Steklov problem. J. Differential Equations 259, no. $5,1778-1818,(2015)$.

[16] V. Burenkov, P. D. Lamberti, Sharp spectral stability estimates via the Lebesgue measure of domains for higher order elliptic operators, Rev. Mat. Complut. 25, 435-457, (2012).

[17] A. Carvalho, S. Piskarev, A general approximation scheme for attractors of abstract parabolic problems. Numer. Funct. Anal. Optim. 27, no. 7-8, 785-829, (2006).

[18] J. Casado-Díaz, M. Luna-Laynez, F. J. Suárez-Grau, Asymptotic behavior of a viscous fluid with slip boundary conditions on a slightly rough wall, Math Models Methods Appl. Sci. 20, 121-156, (2010).

[19] L. M. Chasman, An isoperimetric inequality for fundamental tones of free plates, Comm. Math. Phys. 303, 421-449, (2011).

[20] L. C. Evans, Partial Differential Equations, Graduate Studies in Mathematics, 19. American Mathematical Society, Providence, RI, (1998).

[21] A. Ferrero, F. Gazzola, T. Weth, On a fourth order Steklov eigenvalue problem, Analysis 25, 315-332, (2005).

[22] G. Fichera. Su un principio di dualità per talune formole di maggiorazione relative alle equazioni differenziali. Atti Accad. Naz. Lincei, 19, 411-418, (1955).

[23] F. Gazzola, H.-Ch. Grunau, G. Sweers, Polyharmonic boundary value problems, Springer-Verlag, Berlin, (2010).

[24] F. Gazzola, G. Sweers, On positivity for the biharmonic operator under Steklov boundary conditions, Arch. Rat. Mech. Anal. 188, 399-427, (2008).

[25] A. Girouard, I. Polterovich, Spectral geometry of the Steklov problem, J. Spectr. Theory 7, no. 2, 321-359, (2017).

[26] B. Helffer, Spectral theory and its applications, Cambridge Studies in Advanced Mathematics, 139. Cambridge University Press, Cambridge, (2013).

[27] J. R. Kuttler, Remarks on a Stekloff eigenvalue problem, SIAM J. Numer. Anal. 9, 1-5, (1972).

[28] J. R. Kuttler, Dirichlet eigenvalues, SIAM J. Numer. Anal. 16, 332-338, (1979).

[29] J. R. Kuttler, V. G. Sigillito, Inequalities for membrane and Stekloff eigenvalues, J. Math. Anal. Appl. 23, 148-160, (1968).

[30] P.D. Lamberti, L., Provenzano, Neumann to Steklov eigenvalues: asymptotic and monotonicity results, Proc. Roy. Soc. Edinburgh Sect. A 147, no. 2, 429-447, (2017).

[31] J. Necas, Direct Methods in the Theory of Elliptic Equations, Springer-Verlag, (2012).

[32] L. E. Payne, Some isoperimetric inequalities for harmonic functions, SIAM J. Math. Anal. 1, 354-359, (1970).

[33] O. Post, Spectral analysis on graph-like spaces, LNM 2039, Springer, Heidelberg, (2012).

[34] R. Sperb, Maximum principles and applications, Academic Press, (1981).

[35] W. Stekloff, Sur les problèmes fondamentaux de la physique mathématique, Ann. Sci. Ecol. Norm. Sup. 19, 455-490, (1902).

[36] F. Stummel, Perturbation of domains in elliptic boundary-value problems, in: Lecture Notes Math., Vol. 503, Springer-Verlag, Berlin-Heidelberg-New York (1976), pp. 110-136.

[37] A. F. M. ter Elst, E.M. Ouhabaz, Convergence of the Dirichlet-to-Neumann operator on varying domains. Operator semigroups meet complex analysis, harmonic analysis and mathematical physics, 147-154, Oper. Theory Adv. Appl., 250, Birkhäuser/Springer, Cham, 2015.

[38] G. M. Vainikko, Regular convergence of operators and the approximate solution of equations, Mathematical analysis $\mathbf{1 6}$ (Russian), 5-53, (1979). 
ALBERTO FERRERO,

UNIVERSITÀ DEL PIEMONTE ORIENTALE,

DIPARTIMENTO DI SCIENZE E INNOVAZIONE TECNOLOGICA,

Viale Teresa Michel 11, 15121 Alessandria, ItAly.

PIER DOMENICO LAMBERTI,

UNIVERSITÀ DI PADOVA,

DipARTimento di MATEMATiCA 'TUllio LeVi-CiVitA',

ViA TRIESTE 63, 35121 PADOVA, ITAly.

E-mail addresses: alberto.ferrero@uniupo.it, lamberti@math.unipd.it 